\title{
Direct Numerical Simulation of Transition to Turbulence And Turbulence Control IN PIPE FloW
}

\author{
Dissertation \\ for the award of the degree \\ "Doctor Rerum Naturalium" \\ der Georg-August-Universität Göttingen \\ within the doctoral program GGNB \\ of the Georg-August University School of Science (GAUSS)
}

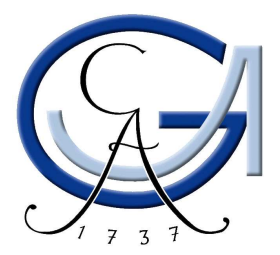

submitted by

BAOFANG SONG

from Henan, China

Göttingen 2014 


\section{Thesis Committee}

Prof. Dr. Björn Hof

Nonlinear Dynamics and Turbulence, Institute of Science and Technology Austria

Prof. Dr. Eberhard Bodenschatz

Fluid Dynamics, Pattern Formation and Biocomplexity, Max-Planck Institute for Dynamics and Self-Organization

Prof. Dr. Jürgen Vollmer

Dynamics of Complex Fluids, Max-Planck Institute for Dynamics and Self-Organization

\section{Member of the Examination Board}

Referee: Prof. Dr. Björn Hof

Nonlinear Dynamics and Turbulence, Institute of Science and Technology Austria

2nd Referee: Prof. Dr. Eberhard Bodenschatz

Fluid Dynamics, Pattern Formation and Biocomplexity, Max-Planck Institute for Dynamics and Self-Organization

\section{Further members of the Examination Board}

Prof. Dr. Jürgen Vollmer

Dynamics of Complex Fluids, Max-Planck Institute for Dynamics and Self-Organization Prof. Dr. Andreas Dillmann

Institute of Aerodynamics and Flow Technology, German Aerospace Center (DLR)

Prof. Dr. Andreas Tilgner

Institute of Geophysics, Georg-August-Universität Göttingen

Prof. Dr. Marc Timme

Network Dynamics, Max-Planck Institute for Dynamics and Self-Organization

Date of oral examination: 29 September 2014 


\section{Abstract}

The subject of this thesis is the transition to turbulence and turbulence control in pipe flow.

In pipes turbulence arises despite the linear stability of the laminar flow (subcritical transition) and directly from onset the flow is spatio-temporally complex. Given sufficiently strong perturbations, turbulence appears in localized patches (puffs) at low Reynolds numbers. At high Reynolds number, patches aggressively grow (slugs) and eventually render the flow fully turbulent. The questions of when and how turbulence starts to grow have long challenged scientists and will be discussed in-depth in this thesis. Turbulence causes higher friction drag and consequently higher energy losses than laminar flow. Control strategies that prevent the formation of turbulence and that relaminarise turbulence are desirable for applications. Some of these strategies were developed in the course of this thesis.

In order to study the transition to fully turbulent flow, the growth of turbulence in terms of the speed of the laminar-turbulent interfaces (fronts) was measured at a variety of Reynolds numbers with highly resolved direct numerical simulations (DNS). The front speed data were compared to experimental measurements from my colleagues and excellent agreement was obtained. These front speeds can be perfectly described by a one dimensional pipe flow model developed by Dwight Barkley, which was inspired by the strong analogy between pipe flow and excitable media (such as nerve axons). A collective effort of theory, DNS and experiments showed that the transition from localized puffs to expanding fully turbulent flow (slugs) is a transition from excitability to bistability. This transition is continuous and a special role is played by nonlinear advection, which masks the tran-

sition point. The nonlinear advection was studied in the DNS and related to the selection of weak or strong downstream fronts.

Based on the transition scenario studied in the first part of the thesis, a forcing strategy was developed to achieve an inverse transition from turbulent to laminar flow. A forcing was used that decelerates the flow near the pipe center and accelerates 
the flow near the pipe wall, modifying the velocity profile into a plug-like one. This modification was found to greatly weaken the turbulence self-sustaining mechanism. In particular it reduces the so-called transient growth (linear streaks amplification) of the flow and is capable of relaminarising turbulence at high Reynolds numbers. A statistical study showed that the minimum transient growth for turbulence to be sustained stays almost constant across a wide range of Reynolds numbers, suggesting that this constant transient growth sets the boundary between excitable and refractory (i.e., unexcitable). By pushing the transient growth below the critical value, pipe flow becomes refractory so that excitation ceases to be sustained and the flow relaminarises. A number of other control strategies have been developed that modify the shear profile.

Another method to relaminarise turbulence is to impose partial slip at the pipe wall. The dynamics of turbulence largely depends on the boundary conditions. The effects of this slip boundary condition on the dynamics of pipe flow turbulence were investigated with DNS. The results showed that azimuthal slip intensifies turbulence, whereas streamwise slip suppresses turbulence. The smallest slip length that suffices to relaminarise turbulence was studied up to Reynolds number $2 \times 10^{4}$ and a linear dependence on Reynolds number was found. While the necessary slip length to achieve relaminarisation is too large to be realized in experiments, the same effect could be achieved by other means, for example by accelerating the fluid near the pipe wall. 


\section{ACKNOWLEDGEMENT}

$\mathrm{PhD}$ is an endeavor! I will never forget the days on which I worked until 1:00am, especially in the last several months. Now I am having a stiff neck and I blame the $\mathrm{PhD}$. It is not something that a man can achieve alone, no matter how hard he/she works, but something that requires supports from MANY others. I am lucky working among very supportive and helpful colleagues and friends. Herein I would like to acknowledge all dear people who helped and are still helping me.

My supervisor Prof. Dr. Björn Hof. What I learned from him is not just science, but more importantly the attitude to science, how to practice science, the way of thinking and explaining ideas etc. He is very responsible. Every time I sent him something I wrote, he always corrected it so much that I even felt a bit frustrated. I indeed improved a lot owing to his strictness and appreciate all the help he offered in and out of work.

Prof. Dr. Marc Avila. I am amazed by that he is always passionate, patient and encouraging. He helped me in every aspect, from career plan and problems on project level to very technical problems regarding writing and programming. His passion is infectious and always cheers me up. It is a great experience working with him.

Prof. Dr. Dwight Barkley. Before the collaboration with him I could not imagine what fluid dynamics can have to do with a nerve cell, but now I know! This work makes me realize further how amazing the nature is.

Dr. Markus Holzner. A great football player and musician. The nice work with him brought me my first paper and for this project I learned parallel programing, which will benefit me forever.

Dr. Ashley P. Willis. For most of my work I use his great pipe flow code. I learned 
high-order difference and spectral methods as well as parallel programing based on his code. I also thank him for patiently reading and correcting one paper draft.

Prof. Dr. Eberhard Bodenschatz and Prof. Dr. Jürgen Vollmer. I thank them for being in my thesis committee and inspecting the progress of my thesis. I learned a lot from the discussion with them during my thesis committee meetings.

The discussion with Dr. Fernando Mellibovsky in Universität Politècnica de Catalunya is acknowledged, and I thank Dr. Haitao Xu for his help since even before I started my $\mathrm{PhD}$.

I thank my colleagues Dr. Sebastian Altmeyer, Giovanni Berterame, Dr. Alberto de Lozar, Jörn Hartung, Dr. Shreyas Vaman Jalikop, Dr. Kerstin Avila, Dr. Jakob Kühnen, Dr. Grégoire Lemoult, Dr. Tobias Lapp, Philipp Maier, Simon Maretzke, Dr. Vasudevan Mukund, Dr. Jose Manuel Gallardo Ruiz, Dr. Nicolas Roland, Dr. Deveranjan Samanta, Jan Schlüter, Dr. Liang Shi, Dr. Paul Steffen, Dr. Xiaoyun $\mathrm{Tu}$, Sascha Warnecke and Dr. Duo Xu. There have been so much fun with them: discussion, sports, eating, drinking and travelling etc.

I thank my friends in Göttingen Xiguang Zhang, Chun Hun, Pengxiang Qiu, Xingyu Ma, Min Xu, Dr. Patrick Say Hwa Tan (now in Singapore), Prof. Hengdong Xi (now in China) and Dr. Xunda Feng (now in the US). They made my life in Göttingen enjoyable instead of being solely about work. I also thank my friends Dr. Chen'an Zhang, Dr. Chunna Li, Ronghui Ma, Ning Wu and Jianfeng Kou in China who have been supporting me.

I acknowledge the financial support from the Chinese Scholarship Council (CSC), Max Planck Institute for Dynamics and Self-Organization (MPIDS) and Institute of Science and Technology Austria (IST Austria). The computing resources from GWDG (Gesellschaft für wissenschaftliche Datenverarbeitung Göttingen), the Jülich Supercomputing Centre and IST Austria are acknowledged.

Last but not least, my families and my girlfriend. Undoubtedly, I can not make it without their love and support. I love them! 


\section{Contents}

Abstract i

Acknowledgement iii

List of Figures $\quad$ ix

Symbols

1 Introduction 1

1.1 The rise of turbulence in pipes . . . . . . . . . . . . . 2

1.1 .1 Turbulent puffs ....................... 5

1.1.2 The onset of sustained turbulence . . . . . . . . . . . . . 8

1.2 Mechanism of turbulence localization . . . . . . . . . . . . . . 9

1.3 The emergence of fully turbulent pipe flow . . . . . . . . . . . . . . . 13

1.4 Turbulence control . . . . . . . . . . . . . . . . . . . . . . 15

1.4 .1 Delay of transition ................... . . 16

1.4.2 Suppression of fully turbulent flow . . . . . . . . . . . . . . 16

1.5 The outline . . . . . . . . . . . . . . . . . . . . 20

2 Numerical methods $\quad 21$

2.1 DNS Code 1: a pressure-free formulation . . . . . . . . . . . . . 22

2.2 DNS Code 2: a primitive-variable formulation . . . . . . . . . . 23

$2.2 .1 \quad$ Formulation . . . . . . . . . . . . . . . . . . 23

2.2 .2 Integration . . . . . . . . . . . . . . . . 24

2.2 .3 The Hagen-Poiseuille flow . . . . . . . . . . . . . 27

2.2.4 Wall friction of fully turbulent flow . . . . . . . . . . . . 28

3 The emergence of fully turbulent flow $\quad 29$

3.1 Speed measurement . . . . . . . . . . . . . . . . . . . . . . . . . 30 
3.1.1 Detection of the fronts and thresholding . . . . . . . . . 30

3.1 .2 Temporal behavior of the front speed, $t_{0}$ and $L_{0} \ldots \ldots 33$

3.1 .3 Speed PDFs . . . . . . . . . . . . . . . . . . 36

3.1 .4 The front speed as a function of $R e$. . . . . . . . . . . . 40

3.2 Modelling puffs and slugs: Barklev's model . . . . . . . . . . . . . . . 42

3.2 .1 The model equations . . . . . . . . . . . . . . . . . . . 43

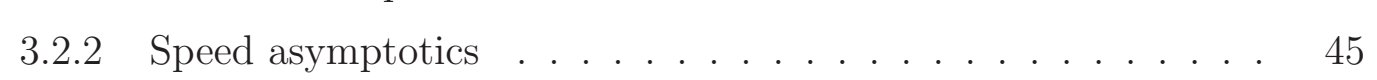

3.2.3 Three types of structure in the asymptotic limit . . . . . . . . 46

3.2 .4 Three types of structure from DNS . . . . . . . . . . . . 48

3.3 Transition scenario in pipe flow . . . . . . . . . . . . . . 49

3.4 Discussion . . . . . . . . . . . . . . . . . . . . . . . 53

4 Turbulence control

4.1 Forcing experiments . . . . . . . . . . . . . . . . . . 57

$4.1 .1 \quad$ Forcing $\ldots \ldots \ldots \ldots \ldots$. . . . . . . . . . . . . . . . . . . . . 57

4.1 .2 Response of fully turbulent flow to the forcing . . . . . . . . 59

4.1.3 Statistical investigation and critical forcing amplitude . . . . . 63

4.1 .4 Linear mechanism interpretation: transient growth . . . . . 65

4.1.5 The control efficiency: energy saving . . . . . . . . . . . . 69

4.1 .6 Localized forcing . . . . . . . . . . . . . . . . . . 71

4.2 Control with streamwise vortices f . . . . . . . . . . . . . 72

4.2.1 Configuration and generation of the vortices . . . . . . . . 73

4.2.2 Deforming the mean velocity profile with vortices . . . . . . 74

4.2 .3 Control with vortices at $\mathrm{Re}=3000 \ldots \ldots$. . . . . . . 76

4.3 Discussion . . . . . . . . . . . . . . . . . . . . 80

$5 \quad$ Slip boundary condition $\quad 83$

5.1 Introduction . . . . . . . . . . . . . . . . . . . 83

5.2 Methods . . . . . . . . . . . . . . . . . . . . 84

5.3 Results . . . . . . . . . . . . . . . . . . . . . . 85

5.4 Discussion . . . . . . . . . . . . . . . . . . . . . . . . . 89

$\begin{array}{lll}6 & \text { Summary } & 91\end{array}$

6.1 Main conclusions . . . . . . . . . . . . . . . . . . . . . 91

6.2 Future work . . . . . . . . . . . . . . . . . . . . . . 94 
$\begin{array}{ll}\text { A Revnolds number reduction } & 97\end{array}$

A.1 Difference between different levels of turbulence $\ldots \ldots \ldots \ldots$. . . . 97

A.2 Implementation of the $R e$ reduction . . . . . . . . . . . . . . . 100

A.2.1 Unphvsical reduction . . . . . . . . . . . . . . . . . . 100

A.2.2 Phvsical reduction . . . . . . . . . . . . . . . . 102

A.3 Discussion . . . . . . . . . . . . . . . . . . . . . . . . 103

B Advection speed of turbulence 105

B.1 Advection speed vs. Re . . . . . . . . . . . . . . . . . . . 107

B.2 Advection speed vs. the centerline velocity . . . . . . . . . 108

B.3 Advection speed vs. the front speed . . . . . . . . . . . . . . . 109

B.4 Discussion . . . . . . . . . . . . . . . . . . . . . . . 110

$\begin{array}{ll}\text { Bibliography } & 113\end{array}$

$\begin{array}{ll}\text { Curriculum Vitae } & 123\end{array}$ 


\section{List OF FiguRES}

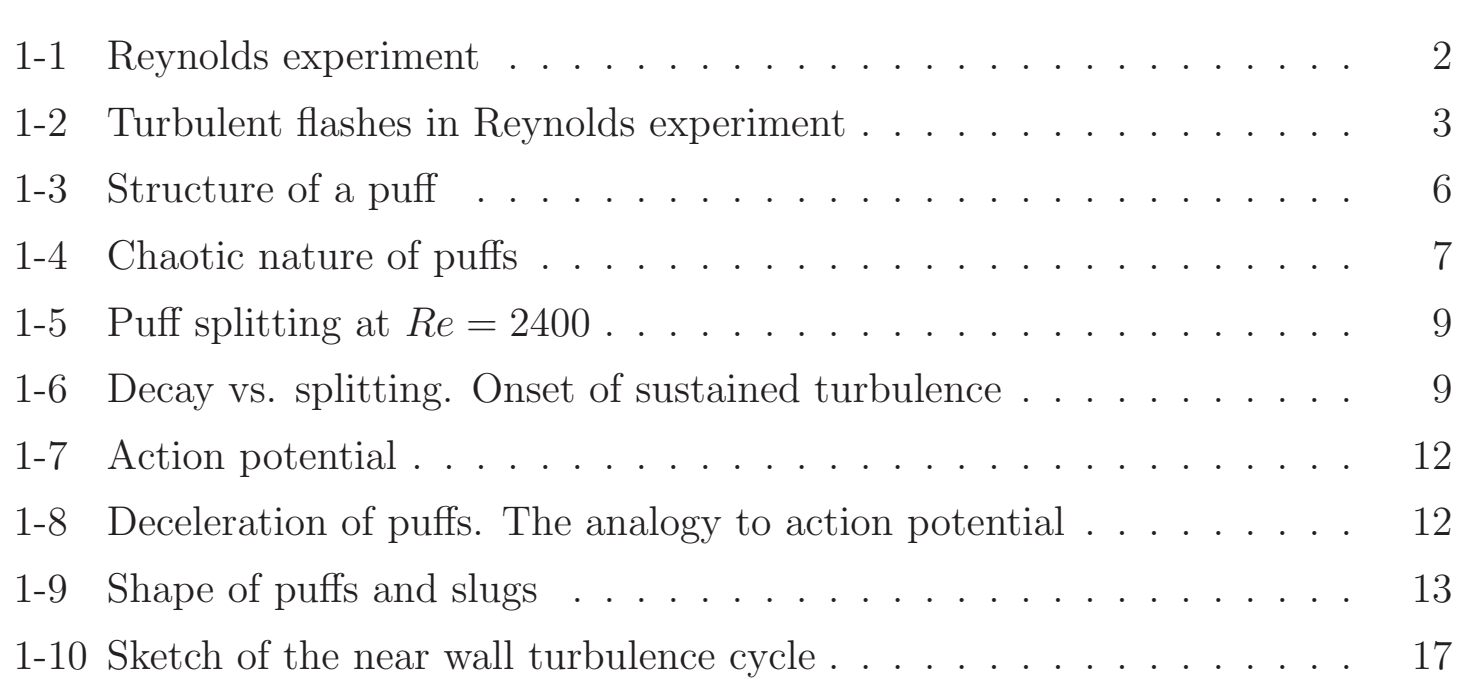

$2-1 \quad$ Development of Hagen-Poiseuille flow . . . . . . . . . . . . . . . 27

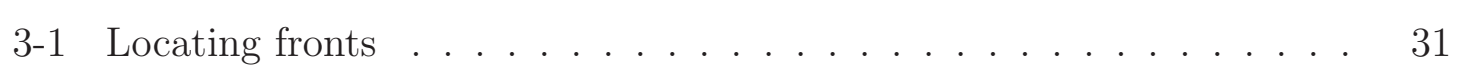

3-2 Thresholding for fronts . . . . . . . . . . . . . . . . . . . . . 32

3-3 Temporal behaviour of the front speed . . . . . . . . . . . . . . 35

3-4 Averaged front speed vs. time . . . . . . . . . . . . . . . 37

3-5 Averaged front speed vs. structure length . . . . . . . . . . . . . . 38

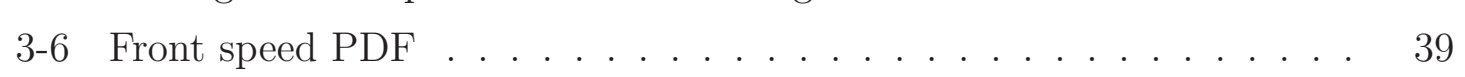

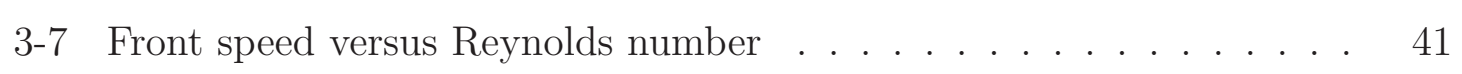

3-8 Growth rate versus Revnolds number . . . . . . . . . . . . . . . . . . 42

3-9 Model nullclines . . . . . . . . . . . . . . . . . . . . . 45

3-10 Fronts in the asvmptotic limit . . . . . . . . . . . . . . 47

3-11 Three types of structure from DNS . . . . . . . . . . . . . . 48

3-12 Front speed from DNS, Experiment and model . . . . . . . . . . . . 50

3-13 Absence of a critical point . . . . . . . . . . . . . . . . 51

3-14 Transient switch between weak and strong fronts . . . . . . . . . . . 53

$4-1 \quad$ Nullclines under forcing $\ldots \ldots \ldots$. . . . . . . . . . . . 56 
$4-2 \quad$ Unforced and forced velocity profiles . . . . . . . . . . . . . . . 58

4-3 The suppression of the force on vorticity . . . . . . . . . . . . . . . . 60

$4-4$ Radial distribution of vorticitv . . . . . . . . . . . . . . . . 60

4-5 Unforced and forced vorticitv contours and velocity fields . . . . . . 61

$4-6 \quad$ Stochastic decav under forcing . . . . . . . . . . . . . . . . . . 62

$4-7 \quad$ Localization under forcing . . . . . . . . . . . . . . . 63

$4-8$ Critical forcing amplitude . . . . . . . . . . . . . . . . . . . 64

4-9 Transient growth under critical forcing . . . . . . . . . . . . . . . . 66

4-10 Transient growth for a puff to be sustained . . . . . . . . . . . . . . . 68

4-11 Energy saving vs. forcing amplitude . . . . . . . . . . . . . . . . . . . 70

$4-12$ Energy saving . . . . . . . . . . . . . . . . . . . . . . 71

$4-13$ Localized forcing . . . . . . . . . . . . . . . . . . . . 72

4-14 Radial velocitv component of the imposed vortices . . . . . . . . . . . 75

4-15 Deforming the mean velocitv profile with vortices . . . . . . . . 76

4-16 Velocitv profile versus wave number and amplitude of vortices . . . . 77

4-17 Control with vortices with wave numbers 2 and $12 \ldots . . . . .78$

4-18 Visualization of vortex control . . . . . . . . . . . . . . 78

$4-19$ Energy spectra $\ldots \ldots \ldots \ldots$

$5-1 \quad$ Azimuthal slip intensifies turbulence f . . . . . . . . . . . . . 86

5-2 Axial velocitv slip dominates the flow . . . . . . . . . . . . . 87

5-3 Relaminarisation under slip boundarv condition . . . . . . . . . . 88

5-4 Critical slip length vs. Reynolds number . . . . . . . . . . . . . . . . 89

A-1 Velocity fields at $R e=3000$ and 10000 . . . . . . . . . . . . . . . 99

A-2 Time series of kinetic energy for Re reduction . . . . . . . . . . . . . 101

A-3 Relaminarisation in case of unphvsical reduction . . . . . . . . . . . 102

A-4 Relaminarisation in case of physical reduction . . . . . . . . . . . 103

B-1 Advection speed vs. time . . . . . . . . . . . . . . . . . . 107

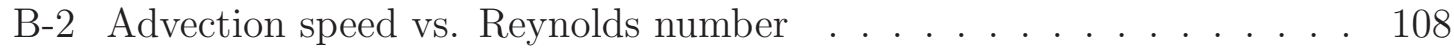

B-3 Advection speed vs. centerline velocity . . . . . . . . . . . . . . . 108

B-4 Advection vs the front speed . . . . . . . . . . . . . . . . . . . 110

B-5 In the frame of reference of turbulence advection . . . . . . . . . . 111 


\section{SyMBOLS}

$\begin{array}{ll}\text { Symbol } & \text { Description } \\ R e & \text { Reynolds number } \\ U & \text { mean flow speed } \\ D & \text { pipe diameter } \\ R & \text { pipe radius } \\ t & \text { time } \\ u & \text { velocity } \\ r, \theta, z & \text { radial, azimuthal and axial coordinates } \\ q & \text { kinetic energy } \iint\left(u_{r}^{2}+u_{\theta}^{2}\right) r d r d \theta \text { in } \\ & \text { pipe flow, and the excitation level in } \\ & \text { Barkley's model } \\ p & \text { pressure } \\ C_{f} & \text { friction coefficient } \\ G & \text { transient growth } \\ E_{c} & \text { kinetic energy } \int_{V}\left(u_{r}^{2}+u_{\theta}^{2}\right) d V \\ L_{z} & \text { pipe length } \\ \beta & \text { forcing amplitude } \\ \lambda & \text { slip length } \\ \omega & \text { vorticity }\end{array}$




\section{CHAPTER 1}

\section{INTRODUCTION}

The motion of fluids can typically take one of two states, laminar flow which is smooth and quiescent, and turbulent flow which is erratic and manifests strong velocity fluctuations, high mixing rates and dissipation. Flows tend to be laminar at low speeds, such as water flows in the vascular systems of plants and blood flows in animals' vessels, and turbulent at high speeds, such as water, gas, and oil flows in pipe-lines. The transition between these two states is one of the central, however, most difficult problems scientists and engineers have been facing in this area. It is still not fully understood even in very simple geometries such as in pipes, channels, and boundary layers.

Shear flow turbulence perhaps is the most common type of turbulence in nature and applications. Pipe, channel, boundary layer, and Couette flows all fall in this class and are usually referred to as 'canonical' shear flows. Pipe flow is probably the most extensively studied amongst these, not least because of its technological relevance. The smooth, steady, and axisymmetric laminar motion in a straight circular pipe was already found by Hagen and Poiseuille in the mid $19^{\text {th }}$ century (Hagen, 1839; Poiseuille, 1840). However, at high flow rates the fluid motion in pipes is usually turbulent and exhibits highly complex spacial and temporal dynamics. The great difficulty of solving the nonlinear partial differential Navier-Stokes equations strongly limited the analytic exploration of the turbulent fluid dynamics. Besides experiments , numerical simulation has become one of the main approaches to turbulence research following the development of computers and efficient numerical algorithms. Especially, the direct numerical simulation (DNS) of turbulence becomes affordable thanks to the high performance computers, and is playing an important role in fundamental turbulence research.

In this thesis, I carried out DNS of pipe flow and studied the transition to fully 
(a)

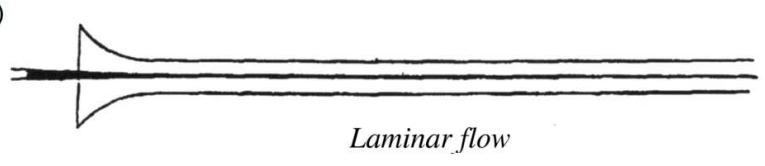

(b)

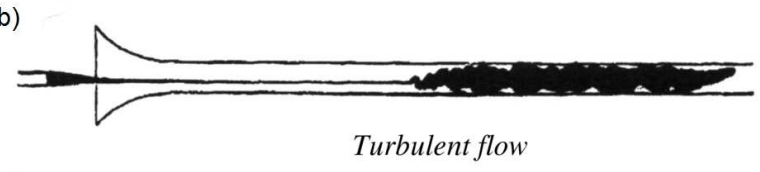

(c)

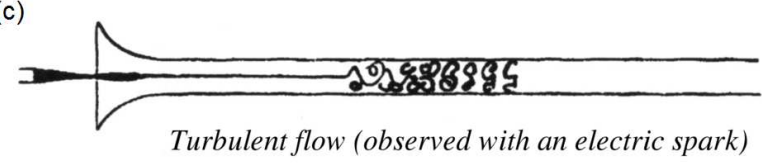

Figure 1-1: Reynolds' 1883 experiment on the transition to turbulence in pipe flow. Flow is from left to right and the ink is injected near the inlet. The shape of the ink illustrates the flow state and vividly shows the changes from steady and smooth laminar state (a) to turbulent state (b). (c) shows a turbulent flow visualized with an electric spark instead of ink as used in (a) and (b).

turbulent flow and explored control strategies that relaminarise turbulence. In what follows, the rise of turbulence in pipe flow will first be briefly summarized. Then the subjects of this thesis, the emergence of fully turbulent flow and turbulence control, will be introduced.

\subsection{The rise of turbulence in pipes}

One central question to be answered is how turbulence arises in simple shear flows. which has been under investigation for over a century, In 1883, Osborne Reynolds published the groundbreaking paper regarding the transition to turbulence and the scaling of frictional drag in pipe flow (Reynolds, 1883a). In his experiment he injected dye into the flow in a straight circular glass tube (see Fig. 1-1) so to visualize the flow. He studied the flow state under different circumstances: various velocities, pipe diameters, and viscosities (water at different temperatures). Reynolds realized that the flow state was determined by the dimensionless group $R e=U D / \nu$, which is now called the Reynolds number. Here $U$ is the mean velocity, $D$ the pipe diameter, and $\nu$ the kinematic viscosity of the fluid (in this thesis all velocity/speed will be normalized by $2 U$, length by the pipe radius $R=D / 2$ and time by $R / 2 U=D / 4 U$,

\footnotetext{
${ }^{1}$ Materials are mainly adapted from the paper Song \& Hof (2014)
} 


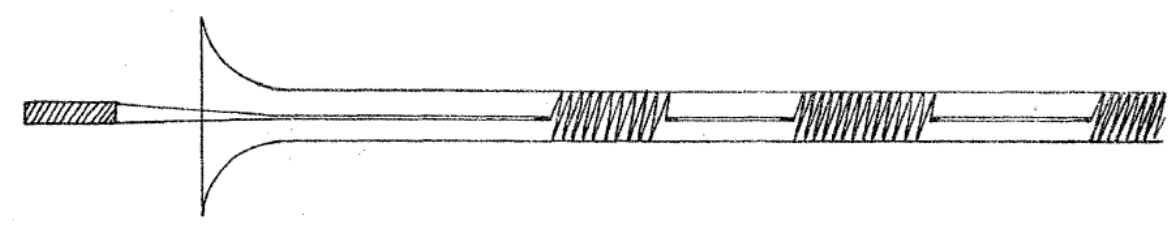

Figure 1-2: Intermittency of pipe flow in the transitional regime observed by Reynolds 1883. He termed the localized patches 'flashes'.

unless explicitly specified). He observed that the dye flows downstream in a straight line when $R e$ is sufficiently low (see Fig. 1-1(a)). When Re was increased to a certain value, the straight and smooth dye streak suddenly broke down and mixed up with the surrounding water (see Fig. 1-1(b,c)), suggesting that at certain Re the transition from laminar flow to turbulence took place abruptly. The other important finding of his experiments was that perturbations above certain magnitude were necessary to trigger turbulence while the flow was stable to smaller disturbances (Reynolds 1883b). In fact, laminar flow can be maintained up to Re as large as 100000 by minimising the inlet flow and background disturbances (Pfenniger, 1961), whereas, turbulence usually occurs at much lower Reynolds number at $R e \simeq 2000$ if the experiment is less controlled. Besides, Reynolds (1883b) also observed that when turbulence first appears it's spatially localized and parts of the flow were still laminar (see Fig. 1-2), a phenomenon nowadays referred to as spatio-temporal intermittency. Following his seminal experimental work, he also attempted to explain the abrupt transition and instability of the flow theoretically but failed (Reynolds, 1895) 2. Despite more than a century of further research the critical Reynolds number $R e_{c r}$ at which the transition to turbulence occurs could only be determined very recently (Avila et al., 2011).

The fact that perturbations with finite amplitude are necessary to trigger turbulence equally applies to other shear flows such as channel flow and Couette flow as well. This fact condemned the attempts of some leading theorists (e.g. Rayleigh, Kelvin, Sommerfeld, Heisenberg, and Lorenz) (Eckert, 2010) to explain the emergence of turbulence by investigating the linear stability of the laminar flow. After many unsuccessful attempts, it became clear (Drazin \& Reid, 1981) that the occurrence of turbulence in these flows is unrelated to the linear stability of the lami-

\footnotetext{
${ }^{2}$ However, in the course he proposed an important method to investigate turbulence by decomposing the flow velocity into a temporal mean and fluctuations with respect to this mean, and subsequently developed the important Reynolds-averaged Navier-Stokes equations (RANS) and the concept of Reynolds stress. Nowadays, the RANS are still the main equations that are used in engineering-oriented computational fluid dynamics.
} 
nar state as Reynolds had already concluded from his experimental observations. It should be pointed out that in Plane Poiseuille flow, i.e., the flow between two plates driven by pressure gradients or constant mass flux, linear instability occurs at about $R e \simeq 5800$ (Orszag \& Kells, 1980), however, turbulence is usually observed far below this Reynolds number at $R e \simeq 1000$ (Davies \& White, 1928; Kao \& Park, 1970). All these results indicate that linear instability is irrelevant for the occurrence of turbulence in these shear flows and the transition in the absence of a linear instability is called subcritical transition.

Two recent theoretical developments provided a plausible scenario for the rise of turbulence in linearly stable flows. In a first stage, because of the non-normality of the linear operator of the linearized Navier-Stokes equations, small perturbations amplify greatly via transient growth before the asymptotic exponential decay (Trefethen et al., 1993; Reddv \& Henningson, 1993; Schmid \& Henningson, 1994; Grossmann, 2000; Meseguer \& Trefethen, 2003). The optimal amplification was found to be through streamwise rolls that generate near wall streaks (Schmid \& Henningson, 1994; Meseguer \& Trefethen, 2000). The amplification could be large such that nonlinear effects are no longer negligible and can potentially trigger turbulence, bypassing the linear instability (Grossmann, 2000). Nonlinear effects must be taken into account when considering how turbulence becomes sustained. Streamwise rolls generate streaks, then the inflectional instability of streaks leads to streak breakdown and nonlinear interaction between streamwise dependent modes regenerate streamwise rolls (the near wall turbulence self-sustaining cycle by Hamilton et al. (1995); Waleffe (1997); Jimenez \& Pinelli (1999)).

In addition, the application of ideas from dynamical systems theory have lead to further insights into this problem. In this perspective, invariant solutions (steady, traveling wave and periodic solutions) to the Navier-Stokes equations are significant for the theoretical description of the transition to turbulence and fully turbulent flow (Kawahara et al., 2012). Since the first exact steady solution for Couette flow by Nagata (1990), many invariant solutions have been found in shear flows (see the reviews by Kerswell (2005); Eckhardt et al. (2007); Kawahara et al. (2012)). These solutions appears as the Reynolds number increases and are all of finite amplitude, and so completely disconnected from the laminar flow. A proposition is that turbulent motion may arise from the instabilities of these solutions (Kerswell, 2005). Besides, these solutions, which consist of quasi-streamwise rolls and streaks, were lately related to the near wall coherent structures in shear flows by Waleffe (1998, 2001, 2003); Itano \& Toh (2001). They are believed to be the building blocks of turbulence that 
organize turbulent dynamics. The dynamical systems approach to turbulence postulates that turbulence can be viewed as a flow winding around and visiting all these invariant solutions (Gibson et al., 2008).

These deep insights into the mechanism underlying the rise of turbulence, however, don't provide an estimate of $R e_{c r}$ (the critical Reynolds number at the onset of turbulence) and a picture of the detailed behaviours of turbulence at the onset. The progress on these aspects have been made mainly via experiments and direct numerical simulations.

\subsubsection{Turbulent puffs}

One important feature of pipe flow is that turbulence first appears surrounded by laminar flow. Reynolds for the first time observed localized structures in the transitional regime at $R e \simeq 2000$ and termed them 'flashes', see Fig. 1-2 (Reynolds, 1883 b). Perhaps the first quantitative investigation to this intermittency is by Rotta (1956). He measured the dependency of the intermittency factor so to determine the critical Reynolds number and claimed that turbulence stays localized up to $R e \simeq 2000$, above which turbulence grows and assuming that for long enough pipes flows would eventually becomes fully turbulent. Wygnanski \& Champagne (1973); Wygnanski et al. (1975) termed the localized structure turbulent 'puffs' and claimed that puffs can not be sustained (decay) below $R e \simeq 2200$ and stay in 'equilibrium' keeping a constant length at $2200 \lesssim R e \lesssim 2300$. The lower limit of the presence of puffs differs in the literature, ranging from about 1700 (Darbvshire \& Mullin, 1995; Avila et al., 2010) to about 2000 (Lindgren, 1969; Wygnanski et al., 1975). Nevertheless, it is a consensus that puffs have a sharp upstream front signaling an abrupt transition from laminar flow to turbulence, and a rather diffusive downstream front where turbulence gradually decays followed by the slow recovery of the velocity profile (Lindgren, 1969; Wvgnanski et al., 1975; Bandvopadhyav, 1986; Darbyshire \& Mullin, 1995; Nishi et al., 2008). Wygnanski \& Champagne (1973), in light of this fact, referred to puffs as 'incomplete relaminarisation'. Fig. 1-3] shows the arrow-headed structure of a typical puff depicted by the kinetic energy of the cross flow $q(z)=\iint\left(u_{r}^{2}+u_{\theta}^{2}\right) r d r d \theta$ (see the red line) and the centerline velocity (the blue line) at the pipe axis. Collectively, these studies showed that turbulent puffs are usually observed at Reynolds numbers $1700 \lesssim R e \lesssim 2300$ and keep an almost constant length while propagating along the pipe roughly at the mean flow speed. Readers are referred to Mullin (2011) for a detailed review on relevant experimental 


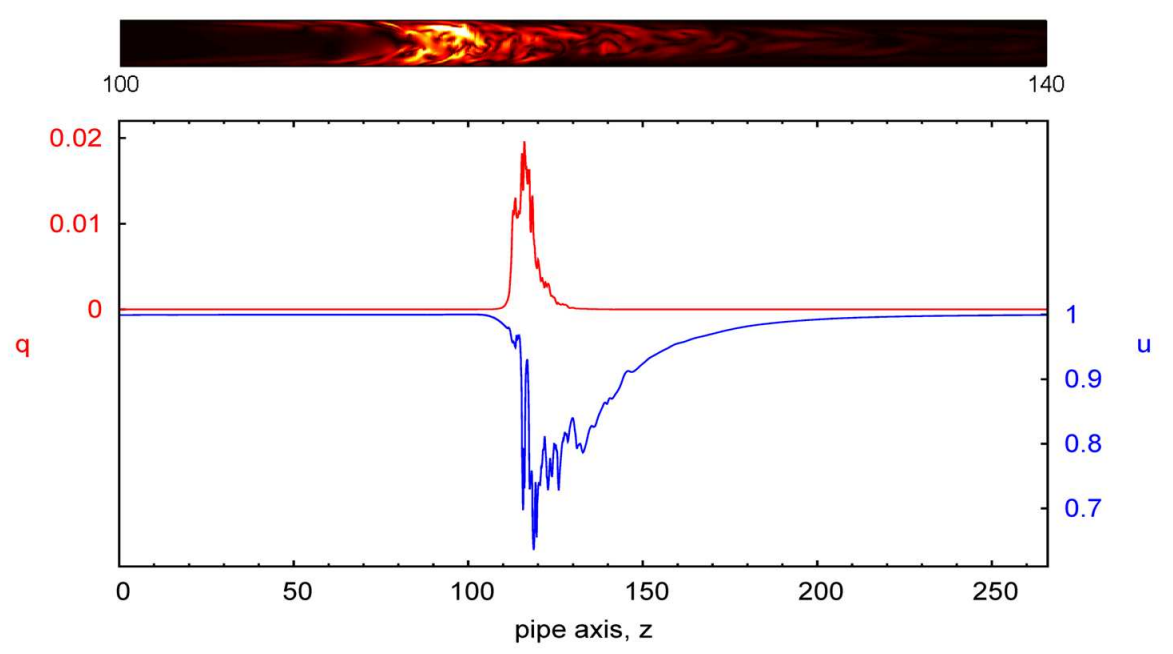

Figure 1-3: The structure of a puff at $R e=2000$. On the top the magnitude of the cross flow $\sqrt{u_{r}^{2}+u_{\theta}^{2}}$ is plotted on the $r-z$ cross section. Only a length of $40 R$ is shown from a $266 R$ pipe. On the bottom, the red line shows the distribution of $q(z)=\iint\left(u_{r}^{2}+u_{\theta}^{2}\right) r d r d \theta$ along the pipe axis for the puff shown on the top. The blue line is the streamwise velocity $u$ at the pipe centerline.

studies.

Recent studies showed that the dynamics of puffs sensitively depends on the initial conditions (Darbyshire1995, Faisst2004). Fig. 1-4 shows this chaotic dynamics. Two runs starting with very close initial conditions (two puffs at $R e=1850$ ) separated by $\sim 10^{-10}$ in velocity depart from each other considerably after evolving independently about 200 convective time units. The separation becomes even more drastic when one of them suddenly relaminarises (see the blue line) at $t \simeq 1200$ while the other persists (the red line). A quantitative measure of this chaotic characteristics, the Lyapunov exponent, was determined in simulations in small periodic domains and shown to be positive (Faisst \& Eckhardt, 2004; Eckhardt et al., 2007), indicating an exponential growth of the separation for two puff trajectories initiated with very close initial conditions. This feature implies that it is impossible to experimentally reproduce a run, instead one should resort to statistical studies for the characteristic behaviours of puffs (Faisst \& Eckhardt, 2004).

More interestingly, both numerical simulations (Brosa, 1989; Faisst \& Eckhardt, 2004) and experiments (Hof, 2004; Mullin \& Peixinho, 2006; Peixinho \& Mullin, 2006) indicated the transient nature of a turbulent puff under $R e \lesssim 2000$, i.e., a puff may stay in 'equilibrium' for a long time before it suddenly decays (see the blue line in Fig. 1-4). Statistical studies showed that the probability that puffs survive up to time $t$ (Faisst \& Eckhardt, 2004), or equivalently up to an observation 


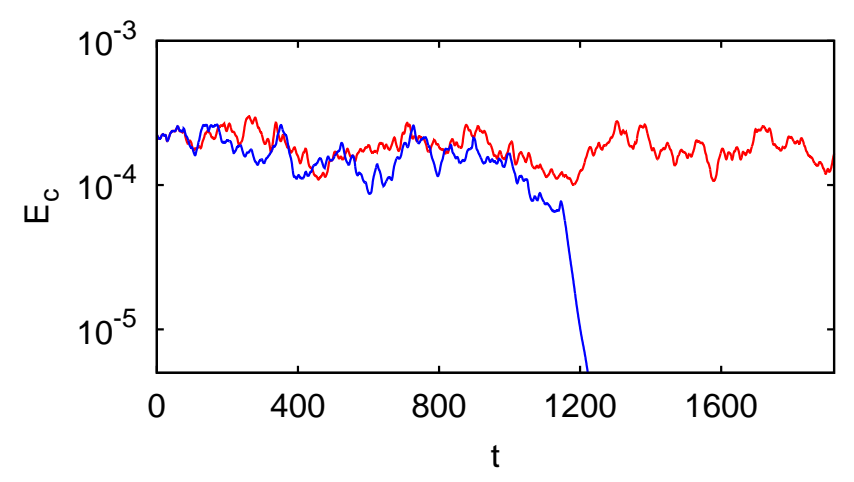

Figure 1-4: Sensitive dependence on the initial conditions of puffs at $R e=1850$ depicted by the the time series of the kinetic energy of the transverse flow $E_{c}=$ $\int_{V}\left(u_{r}^{2}+u_{\theta}^{2}\right) d V$ of two runs. These two runs are initiated with very close initial conditions separated by $\sim 10^{-10}$ in velocity while they depart from each other quickly after about 200 time unit. One of them decayed abruptly at $t \simeq 1200$ (the blue line).

position along a pipe (Peixinho \& Mullin, 2006), follows an exponential distribution at a given Reynolds number, a signature of memoryless processes. Given such a distribution, the mean lifetime of puffs can be extracted. The scaling of this mean lifetime $\tau(R e)$ with Reynolds number has been the subject of both experimental studies (Mullin \& Peixinho, 2006; Hof et al., 2006, 2008) and numerical studies (Eckhardt \& Faisst, 2004; Willis \& Kerswell, 2009; Avila et al., 2010). There are no definite theoretical predictions of this scaling (Eckhardt et al., 2007), and has been mainly studied by experiments and simulations. It was a consensus that the lifetime of a single puff increases as Re increases. While a main debate among these studies was whether the lifetime of a single puff will diverge at a finite $R e$ so that turbulence gets sustained or not. Conflicting opinions were held regarding this question. Faisst \& Eckhardt (2004) in numerical simulations found that the mean lifetime of puffs diverges at $R e \simeq 2250$, indicating the onset of sustained turbulence at finite $R e$. Later experimental results by Mullin \& Peixinho (2006); Peixinho \& Mullin (2006) supported this divergence of the mean lifetime and suggested $\tau \sim 1 /\left(R e_{c r}-R e\right)$, however, gave a different critical Reynolds number about $R e \simeq 1750$. Willis \& Kerswell (2007) studied the lifetime with DNS in a periodic pipe considerably longer than a puff, about 50D, and also supported the $\tau \sim 1 /\left(R e_{c r}-R e\right)$ scaling with a $R e_{c r}=1870$. On the other hand, Hof et al. (2006) questioned the above claims by both experiments in very long pipes $(7500 D)$ and numerical simulations (in a $5 D$-long pipe) and argued that the lifetime of puffs scales exponentially with the Reynolds number as $\tau \sim \exp (c R e)$ for some constant $c$. This is a clear indicator that a single puff stays transient at any Reynolds number hence, never becomes sustained. In even longer 
pipes, Hof et al. (2008) found that the lifetime increases super-exponentially with the Reynolds number. Willis \& Kerswell (2009) argued that the pipe in the simulations of Hof et al. (2006) was too short to contain a puff and the lifetime was affected by the limited domain size. With a reduced numerical model, they studied the effect of the pipe length on lifetime statistics and claimed that turbulence always stays transient in short pipes, however, undergoes a divergence in lifetime and becomes sustained at some $R e$ in pipes longer than $\sim 50 D$. Nevertheless, Avila et al. (2010) improved the statistics of Willis \& Kerswell (2009) using larger sample sizes and observation times and demonstrated the super-exponential scaling found by Hof et al. (2008). This scaling has been later confirmed in experiments by Kuik et al. (2010). Goldenfeld et al. (2010) related the transition to turbulence to extremal statistics and theoretically reproduced the super-exponential behaviour of the lifetime of puffs.

The transient nature of localized turbulence is clearly at odd with the classic picture of turbulence of a chaotic attractor (Ruelle \& Takens, 1971). This was questioned much earlier by Crutchfield \& Kaneko (1988), who suggested a 'long transients' nature of turbulence. Instead, the dynamics of puffs can be better related to a chaotic saddle, which has unstable directions in which the chaotic dynamics can escape and revert to the resting state, i.e., the laminar flow (Faisst \& Eckhardt, 2004; Eckhardt et al., 2007).

\subsubsection{The onset of sustained turbulence}

Lindgren (1969); Wygnanski \& Champagne (1973); Nishi et al. (2008) observed that, localized turbulence tends to grow and split into more puffs above $R e \simeq 2400$. Fig. 1-5 shows the splitting process of a puff at $R e=2400$. At the downstream front vortical structures are shed downstream. Usually they decay but occasionally they may escape far enough to seed another puff, which is called puff splitting. Moxey \& Barkley (2010) proposed that turbulence becomes sustained by this spatial proliferation at $R e \simeq 2300$ (puff splitting). Interestingly, puff-splitting is also a memoryless process and can be observed at $R e$ much lower than what former studies reported (Avila et al. 2011). Similar statistics as that of lifetimes unveiled the super-exponential decrease of the characteristic time for puff splitting with Reynolds number. A simple picture was then obtained: the competition between the transient nature and the splitting nature of puffs determines when turbulence becomes sustained. If splitting occurs on a shorter time scale than puff decay, turbulence will tend to proliferate rather than decay, and it will be sustained in the thermodynamic limit when the system size is 


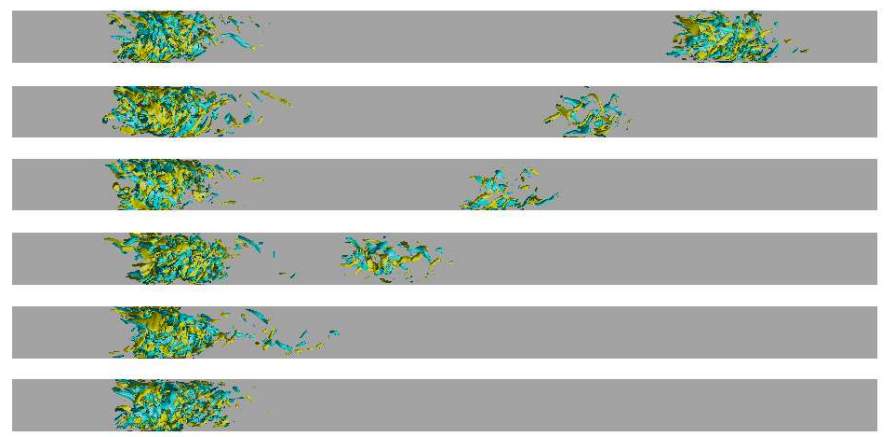

Figure 1-5: The splitting process of a puff at $R e=2400$. The isosurfaces of the streamwise vorticity are plotted, and yellow and cyan represent \pm 1.0 of the streamwise vorticity. Time goes up and panels are separated by 80 time units.

large. The intersection point of the time scales of these competing processes pins the critical Reynolds number at $R e_{c r}=2040$, at which the onset of sustained turbulence occurs (Avila et al., 2011).

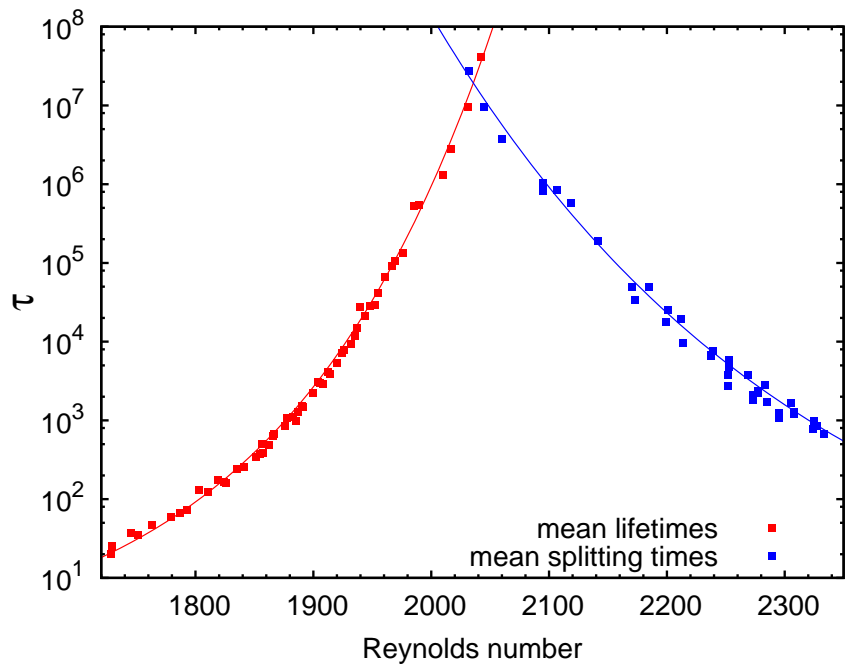

Figure 1-6: The onset of sustained turbulence determined by the competition of puff decay and splitting. Symbols are DNS and experimental data from Hof et al. (2008); Avila et al. (2011) and lines are super-exponential fitting. The intersection point is at $R e=2040$. To the right of this point, splitting occurs on a shorter time scale than puff decay so that turbulence becomes sustained via this spatio-temporal intermittency.

\subsection{Mechanism of turbulence localization}

Besides pipe flow, localized turbulent states were also found in other shear flows if initiated with finite-amplitude perturbations. For example, turbulent stripes tilted 
with respect to the streamwise direction in Couette flows (Bottin \& Chaté, 1998; Bottin et al., 1998; Barkley \& Tuckerman, 2005; Duguet et al., 2010a), and turbulent spot in channel and boundary layer flows (Emmons, 1951; Wygnanski et al., 1976; Henningson et al., 1987). In fact, spatially localized states are also found in other dissipative systems. These include spiral waves and target patterns in chemical reactions (Zaikin \& Zhabotinskii, 1970; Winfree, 1972; Müller et al., 1985) and in cardiac tissues (Davidenko et al., 1992; Cherrv \& Fenton, 2008), action potential on nerve axon (Hodgkin \& Huxlev, 1952; FitzHugh, 1961; Nagumo et al., 1962), spreading of epidemics (Murray et al., 1986), binary fluid convection (Niemela et al., 1990; Batiste et al., 2006), ferrofluid under magnetic fields (Richter \& Barashenkov, 2005), and even nonlinear optics (Tlidi et al., 1994, 1997). For a discussion on localized states in dissipative systems see (Knobloch, 2008) and references therein, and for a review on the theoretical treatment of wave propagation and wave fronts in excitable medium see (Tyson \& Keener, 1988).

In pipe flow localization takes the form of puffs at low Reynolds numbers. A feature of puffs is the asymmetry in upstream and downstream edges: a sharp upstream front while a not well-defined downstream front (see the sharp upstream front on the left and the diffusive downstream front on the right in Fig. 1-3). Wygnanski et al. (1975) gave a physical explanation on the localization of puffs based on an energy budget analysis. The parabolic velocity profile upstream of the trailing edge is able to support strong turbulence, however, the plug-like velocity profile inside the puff suppresses the energy production due to a mismatch in the location of large radial velocity gradient and the Reynolds stress. Consequently, turbulence keeps decreasing following the upstream front and the flow gradually relaminarises downstream, resulting in a less well-defined diffusive downstream front. Following the relaminarisation, the velocity profile gradually relaxes to a parabola. A recent study (Hof et al., 2010) explicitly showed that a puff feeds on the adjacent laminar shear profile upstream of its upstream front. If the upstream laminar flow is modified and replaced by a plug-like profile, the puff decays. Equally if two puffs are too close to each other, the upstream puff will modify the laminar profile adjacent to the downstream puff into a plug-like profile, and consequently eliminate the downstream puff (evidenced by Hof et al. (2010)). As a consequence, there is a puff-puff interaction distance, below which the downstream puff will not survive. This distance was shown to be about $20 D$ (Samanta et al. , 2011). The same mechanism is also present in other shear flows such as duct flow and channel flow (Hof et al., 2010$)$.

An interesting analogy between pipe flow turbulence and phenomena in excitable 
media such as the action potential of a nerve axon, was realized by Barkley (2011b). Excitable systems have a spatially extended resting (quiescent) state that will persist without perturbations. However, perturbed above a certain threshold, there will be an excited state propagating through the system. For a nerve axon, the resting state is a negatively polarized cell membrane. If stimulated sufficiently with an electric current, a localized excitation will propagate along the axon. After being excited, the membrane is depolarized and will not be able to support excitation anymore, and subsequently the excitation decays. The polarization will gradually relax to the resting state with the absence of excitation, and the membrane regains the susceptibility to excitation, when the polarization has sufficiently recovered. The generic features of excitable media can be summarized as (for more details see Tyson \& Keener (1988)):

- a unique spatially extended resting state (e.g. fully polarized cell membrane);

- small perturbations damp out but suprathreshold perturbations can excite the system (e.g. by sufficiently strong electric current);

- a recovery variable, or a controller (e.g. membrane potential);

- a trigger variable, or a propagator, whose level is controlled by the recovery variable (e.g. ionic conductance by membrane potential);

- a refractory period (or region) following an excitation within which excitation decays. The excited area returns to the resting state after a (slow) recovery process.

- the localized state can only maintain itself via the trigger front continuously exciting the unperturbed region on one side.

Fig. 1-7 illustrates such features of (one dimensional) excitable media. The generic dynamics of such kind of systems has long been studied using low-dimensional model equations. For example, the FitzHugh-Nagumo model (FitzHugh, 1961; Nagumo et al., 1962) for the action potential of a nerve axon (Hodgkin \& Huxley, 1952).

Turbulent puffs bear a surprising analogy to the nerve axon mentioned above. Laminar pipe flow (the resting state) can be excited by finite-amplitude perturbations to an excited state (puff). A sharp trigger front continuously excites laminar flow at the upstream front of puffs. The velocity profile will be disturbed such that the profile is plug-like. This plug-like profile can not support turbulence, so that turbulence decays downstream of the trigger front and gradually flow relaminarises. The velocity profile slowly recovers towards a parabola as turbulence decays and it does not support new excitations before it has sufficiently recovered (the refractory 


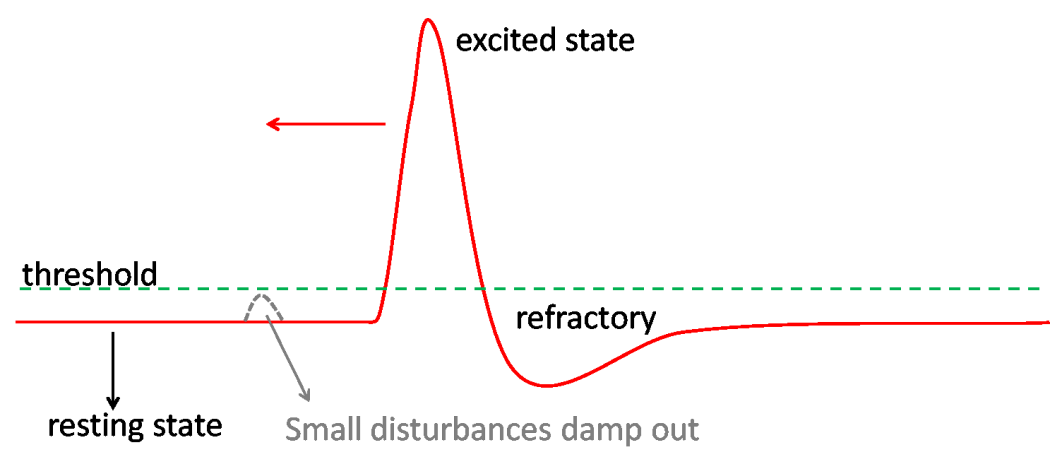

Figure 1-7: The generic features of excitable media exemplified by the action potential (the depolarization of the cell membrane) of a nerve axon. Small perturbations damp out (see the gray dashed line), and suprathreshold ones excite the system to an excited state followed by a refractory period (region) within which excitation decays and system relaxes to the resting state.

period). Fig. 1-8 together with Fig. [1-7] illustrate the analogy between pipe flow and excitable media (e.g. action potential of a nerve axon). In Fig. 1-8 the deceleration

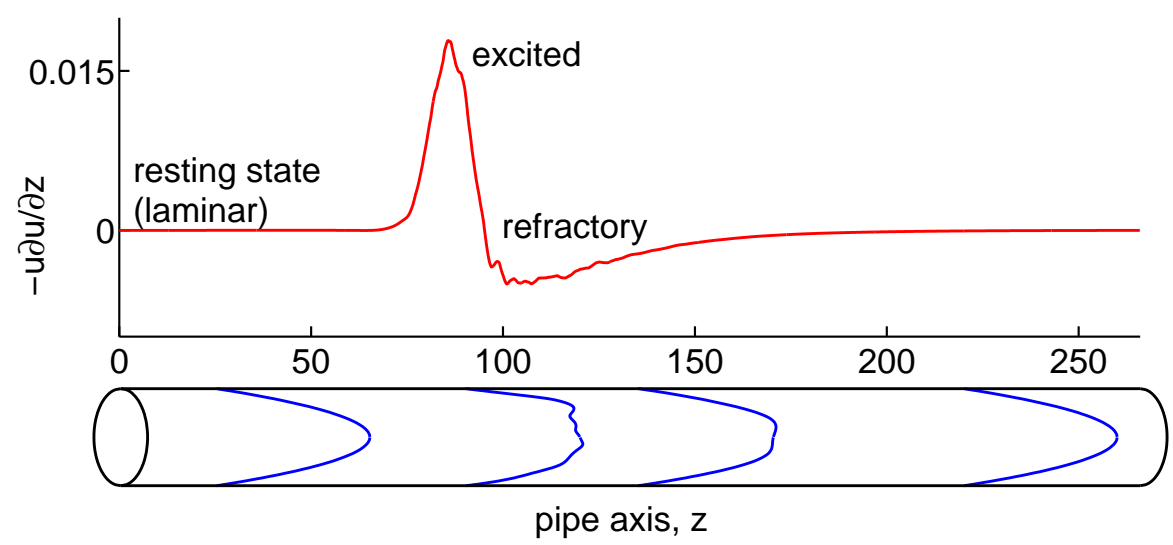

Figure 1-8: The deceleration of a puff at the pipe centerline (the red line). On the bottom the velocity profile at several positions in the pipe are plotted showing the unperturbed profile (left one) and the recovery following the excitation (the three on the right). The aspect ratio of the pipe is changed for the purpose of visualization

at the pipe centerline is plotted, which resembles the shape of the action potential in Fig. 1-7. Below, the velocity profile of the resting state (laminar flow), of the excited state (puff), inside the refractory region, and the recovered profile are compared.

This analogy suggests to view pipe flow in the context of excitable media and to model pipe flow with equations with low order nonlinearities (Barkley, 2011b). An apparent difference is that the self advection of turbulence in pipe flow is clearly absent in the action potential models for a nerve axon. Besides, there is no parameter 
corresponding to the Reynolds number in the models, so that no transition of the dynamics has been discussed in there. Barkley's model incorporated these two factors and for the first time suggested the transition from puffs to slugs is a transition from excitability to bistability (Barkley, 2011b).

\subsection{The emergence of fully turbulent pipe flow}

Turbulence is usually initiated with localized perturbations, which will develop into turbulent patches. Localized turbulence becomes sustained via puff splitting above the critical Reynolds number 2040, however, turbulence still stays localized in the vicinity of the onset of sustained turbulence. At higher Reynolds numbers (above $\sim 2800$ ), another type of structure was observed, slugs, which have a very different structure and behaviour from puffs (Rotta, 1956; Wygnanski \& Champagne, 1973; Wygnanski et al., 1975); Darbyshire \& Mullin, 1995). Slugs were observed to have both well-defined upstream and downstream fronts. Once formed, they will continuously grow and eventually fill arbitrarily long pipes. Fig. [1-9]shows a puff at $R e=2000$ and a slug at $R e=4500$ in a $L_{z}=360$ pipe. The differences in the structure and downstream front are apparent. The slug has a spatially extended plateau in both the turbulence intensity and centerline velocity in the core region, while the puff has none of them. It indicates that the interior part of slugs is in a stabilized turbulent state. Besides, the slug has a well-defined downstream front which looks similar to its upstream front. Wygnanski et al. (1975) showed that the interior of slugs is the

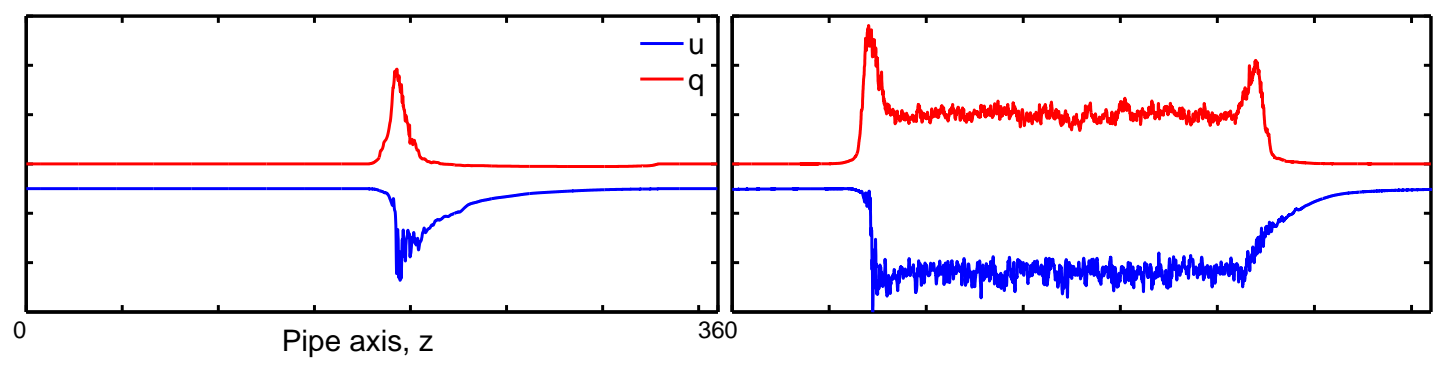

Figure 1-9: The different structures of a puff at $R e=2000$ (left) and a slug at $R e=4500$ (right). The local turbulence intensity $q$ and the centerline velocity $u$ are plotted.

same as fully developed turbulence and considered slugs as the predecessor of fully turbulent flow, while they called puffs an incomplete relaminarisation.

The development from localized structures to fully turbulent flow can be illustrated by the speed of the upstream and downstream fronts (also referred to as trail- 
ing and leading edges in the literature). In attempts to study the transition to fully turbulent pipe flow, the front speed has been studied at a wide range of Reynolds numbers (Lindgren, 1969; Wvgnanski \& Champagne, 1973; Sreenivasan \& Ramshankar, 1986; Durst \& Ünsal, 2006; Nishi et al., 2008; Duguet et al., 2010b). Speed measurements gave rough agreement among the several data sets. Collectively, these speed measurements showed that turbulence approximately starts to grow at about $R e=2300 \sim 2500$, and the downstream front speed smoothly changes from the value for a puff to that for a slug. No sharp transition and clear critical point were observed. Sreenivasan \& Ramshankar (1986) claimed a square root scaling of the growh rate with the distance to a 'critical' Reynolds number at which the growth starts, and a later study supported this claim (de Lozar \& Hot, 2010).

Besides performing speed measurements, Lindgren (1969) estimated the upstream front speed based on energy considerations, but the agreement between the two was poor. Wygnanski \& Champagne (1973); Wygnanski et al. (1975) gave much more detailed properties of the fronts of slugs and puffs based on an energy budget analysis and stressed the difference in their downstream fronts. It may be worth mentioning that two different kinds of slug seem to be observed by (Duguet et al., 2010b), yet this has never been stressed in the literature.

In fact, because of the complex interval between puff and slug regimes and the intrinsic turbulent fluctuations which create holes even in growing turbulence at relatively high Reynolds numbers (around 2700 2800) (Avila \& Hof, 2013), a well defined onset of slug (continuous growth of turbulence) is still lacking in measurements, let alone the mechanism that gives rise to the growth. The lack of a critical scaling or a clear critical point makes the transition to fully turbulent flow still poorly understood after decades of research.

Inspired by the analogy between puffs and action potential of a nerve axon discussed in Section 1.2, Barkley (2011b, a) developed two-variable one-dimensional models (both continuous and discrete) that successfully capture some large-scale behaviours of puffs (decay and splitting) and of slugs. The continuous model nicely depicts the bifurcation scenario that gives rise to the appearance of slugs and allows to view the transition from puffs to slugs as a transition from excitability to bistability. It should be pointed out that, Barkley's models for the first time provide a framework in which the problem of transition to fully turbulent flow may be tackled. The fluctuations, which are intrinsic to turbulence but may cover the most fundamental dynamics, are absent in the PDE model. This fact allows the model to give a clear bifurcation scenario as Re increases. However, the model failed to pre- 
dict correct behaviours of the fronts documented in the literature 3 (Lindgren, 1969; Wygnanski \& Champagne, 1973; Durst \& Ünsal, 2006; Nishi et al., 2008).

With an attempt to improve the model and to put forward the understanding of the onset of fully turbulent flow, I carried out extensive DNS of puffs and slugs and accurately computed the front speeds. Besides, a detailed study on the dynamics of the fronts was carried out. Together with experimental results from colleagues (by Mukund Vasudevan), the data allowed a new model by Barkley to capture the essential behaviour of turbulent fronts in all flow regimes. Finally, a deep understanding of the emergence of fully turbulent flow has been obtained (Barkley et al., 2014).

\subsection{Turbulence control}

One important feature of turbulence is its high energy dissipation compared to laminar flow. As a consequence, the friction (drag) is higher in case of turbulence than for laminar flow. In the latter, the friction factor decreases with Reynolds number as $C_{f} \propto R e^{-1}$, whereas $C_{f} \propto R e^{-1 / 4}$ in fully turbulent flow. For example, in water pipelines with diameter $50 \mathrm{~cm}$ and an average speed of $1 \mathrm{~m} / \mathrm{s}$, the Reynolds number is about $5 \times 10^{5}$. The friction loss and hence the pumping costs are almost 100 times larger for turbulent than for laminar flow. Although in pipelines the laminar flow state is linearly stable at all Reynolds numbers, in practice all flows at high $R e$ are fully turbulent because of the unavoidable disturbances.

The high friction drag caused by turbulence does not only causes much higher drag in pipes, but also causes much higher energy consumption of aircraft and ships than if the flow were laminar. Effective turbulence control methods are hence of huge interest for practical applications and could lead to very large energy saving. The development of control strategies relies on the in-depth investigation into both the emergence of fully turbulent flow and the self-sustaining mechanism of wall shear turbulence.

Over the years a variety of control strategies have been developed for wall shear flows and drag reduction has been achieved. These roughly can be categorized into passive and active controls. Passive control includes wall roughness and riblets (Michael \& Walsh, 1983; Bechert et al. 1997; Sirovich \& Karlsson, 1997; Fransson et al., 2006), active control includes open-looped boundary blowing and suction control (Antonia et al., 1988; Sumitani \& Kasagi, 1995), transverse wall movement (Choi et al.,

\footnotetext{
${ }^{3}$ private communication with Dwight Barkley
} 
2002; Quadrio et al., 2009), optimal and suboptimal control (Choi et al., 1993, 1994; Lee et al., 1998; Bewlev et al. , 2001; Högberg et al., 2003), and linear model-based feedback control (Bewlev et al., 2001; Högberg et al., 2003; Moarref \& Jovanovic, 2010; Lieu et al., 2010; Semeraro et al., 2013). Readers are referred to Collis et al. (2004); Gad-el Hak (1996); Bewley (2001); Kim (2003); Kim \& Bewley (2007); Kasagi et al. (2009) for comprehensive references. From the point view of the drag-reduction mechanism, these strategies fall in two approaches: delay (or prevent) the transition to turbulence by suppressing the growth of initial disturbances, and suppress fully turbulent flow by interrupting its self-sustaining process

\subsubsection{Delay of transition}

One approach to turbulence control is to suppress or delay the energy growth of initial perturbations so to subdue the transition to turbulence. Both passive and active control strategies have been proposed. Fransson et al. (2006) proposed a passive control mechanism using wall roughness that generates moderate streaks in the boundary layer, which in turn stabilize the flow and delays the breakdown to turbulence. Moarref \& Jovanovid (2010); Lieu et al. (2010) developed open-looped modal-based control strategies in channel flow that delay the onset of transition with well-designed downstream traveling waves via boundary blowing/suction (active control). Feedback (active) control strategies that delay the linear stage of energy amplification are mostly based on control theory combined with linearized Navier-Stokes equations (for a review on this topic see Kim \& Bewley (2007)). For example, Semeraro et al. (2011, 2013); Belson et al. (2013) designed active feedback control strategies to suppress the energy growth of both 2- and 3-dimensional disturbances in boundary layer flow with sensors and actuators in combination with reduced-order models. However, very often we have to deal with fully turbulent flow in the first place in real applications due to prevailing disturbances and the low disturbance threshold for triggering turbulence at high Reynolds numbers.

\subsubsection{Suppression of fully turbulent flow}

An alternative is to suppress turbulence or even relaminarise fully turbulent flow. One class of control is a systematic approach based on modern control theory such as optimal feedback control (Bewley et al., 2001; Högberg et al., 2003; Kim \& Bewley, 2007). These authors achieved drag reduction and even relaminarised fully turbulent channel flow. However, those control strategies require the flow information in the in- 
terior of the flow domain for the optimization, which are usually not easily measurable in experiments. Ideally, practical control system should rely on minimal information from the flow, such as quantities that can be measured on the wall.

Another class of control strategies do not rely on rigorous control theory, instead, rely on the knowledge of the self-sustaining mechanism of wall shear turbulence, which has been extensively studied in recent decades but is still not fully understood. It was found that the near wall region is characterized by coherent structures, i.e., low speed and high speed streaks, quasi-streamwise vortices (Kline et al., 1967; Kim et al., 1987; Robinson, 1991). Quasi streamwise vortices generate streaks by convecting the mean shear, however, the vortices regeneration mechanism is still not completely clear. Through studies of the minimum turbulence unit (Jimenez \& Moin, 1991), which greatly reduces the complexity of the turbulence whilst keeping the main features of the turbulent dynamics, Hamilton et al. (1995); Waleffe (1997); Jimenez \& Pinelli (1999) proposed a near wall turbulence self-sustaining cycle and argued that the quasi streamwise vortices are generated by the instability of low speed streaks. Fig. 1-10 illustrates this wall cycle. Schoppa \& Hussain (2002) argued that the normal-mode

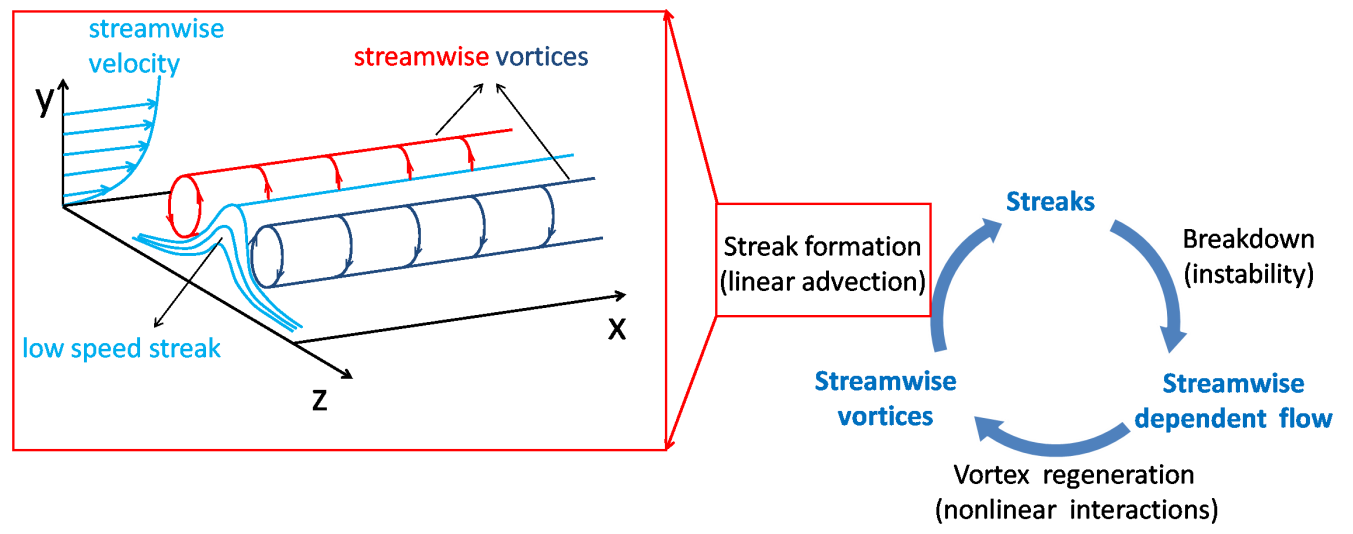

Figure 1-10: The near-wall turbulence self-sustaining cycle proposed by Hamilton et al. (1995); Waleffe (1997) (the cycle on the right). The process of streak formation is sketched on the left in the red frame.

instability of the streaks does not contribute significantly to vortex generation and instead a linear streak transient growth mechanism is responsible for the amplification of the streamwise dependent perturbations and streamwise vortices regeneration. Berhard et al. (1993); Brooke \& Hanratty (1993); Zhou et al. (1999) proposed instead a parent-offspring mechanism in which the near wall vorticity sheet induced by parent (hairpin) vortices directly generates (hairpin) vortices. Collectively in these models, however, it is clear that the quasi-streamwise vortices extract energy from 
the mean shear to feed the near wall structures and are responsible for the high drag and Reynolds stresses in turbulence (Kim et al., 1987).

Control on vortices: wall blowing/suction. In light of the important role that streamwise vortices play in the drag and shear stress production, Choi et al. (1994) proposed an opposition control technique which aims at counteracting the wall normal velocity components (of streamwise vortices) by blowing/suction at the wall and achieved drag reduction about 25\%. However, this control needs the information inside the flow at $y^{+}=10$ which is not easily available in experiments. Similarly, Kim \& Lim (2003) claimed that a linear mechanism that couples wall normal velocity and wall normal vorticity is essential for generating the wall-layer streaks, which in turn are essential for regeneration of vortices. They found that without this coupling mechanism turbulence can not sustain itself. They suggested to suppress turbulence with proper opposition control (Choi et al., 1994) so to minimize this coupling effect. Nevertheless, it also requires the information inside the flow for the optimization. To avoid these restrictions, Lee et al. (1998) proposed a numerical suboptimal control strategy that only requires the pressure or shear stress on the wall. This strategy can be implemented with wall blowing/suction and was shown to lead to $16 \sim 22 \%$ drag reduction in channel flow. Xu et al. (2002) applied suboptimal opposition control to pipe flow at $R e_{\tau}=150$ and achieved drag reduction about $13 \%$ to $23 \%$ in DNS. With the development of micro-electromechanical systems (MEMS), these control techniques that only require the information on the wall can be implemented in experiment using small scale sensors/actuators on the wall (Kasagi et al., 2009). Unfortunately, in experiments drag reduction has barely been achieved with MEMS (Kasagi et al., 2009).

Control on streaks. Besides controls that act on the wall normal velocity (so that on vortices), another type of control is based on the streak-instability vorticesregeneration mechanism. Efforts have been taken in weakening or stabilizing the near-wall low-speed streaks which were found to be inflectionally unstable, generating new streamwise vortices following their breakdown. Du \& Karniadakis (2000); Du et al. (2002) showed that near-wall streaks can be eliminated by means of a transverse traveling wave, and that low-speed streaks can be weakened by a spanwise flow oscillation. Numerically their traveling waves and oscillation were simulated by forcing the flow in spanwise direction within the sublayer, and experimentally they used an array of electromagnetic tiles (permanent magnets and electrodes) distributed on the wall in conductive salt water. They claimed substantial net energy gain with this technique. However, it is still a challenge to implement sensors/actuators-based 
control on very small length scales in engineering applications, and large scale control is desired (Schoppa \& Hussain, 1998).

Iuso et al. (2002); Schoppa \& Hussain (1998) respectively tested large-scale vortices control experimentally and numerically in channel flow. In experiment streamwise vortices were generated by injection from the wall and numerically by forcing. They respectively reported about $30 \%$ and $20 \%$ reduction in skin friction and Schoppa \& Hussain (1998) even observed up to $50 \%$ skin friction reduction in their simulation with wall jet collision control. They attributed the skin friction reduction to the reorganizing and stabilizing effect of the large scale vortices on the low speed streaks and the attenuation of the streamwise vortex formation in the near wall region. Willis \& Hwang (2010) also found a possibility to reduce drag by forcing the large scale streaks in pipe flow and reported a power saving up to $11 \%$. However, to my knowledge, so far there is no report of relaminarisation of fully turbulent flow with large scale controls.

Control on the velocity profile. Another possible approach is to develop large scale control methods that target the velocity profile because it is the energy source in near wall turbulence. If this energy supply can be subdued then turbulence will certainly decay or even relaminarise. The velocity profile also is a crucial factor in the linear-coupling mechanism suggested by Kim \& Lim (2003), which they argued is essential for fully turbulent flow sustaining itself. It was also shown that a change in the mean shear (or mean velocity profile) causes a great change in the energy balance in channel flow (Tuerke \& Jimenez, 2013). Although these authors did not report relaminarisation, their results imply that by forcing the mean velocity profile to an unnatural shape the energy balance may be perturbed so much that relaminarisation occurs. These findings are consistent with the observation in pipe flow in the transitional regime (Hof et al., 2010). These authors argued that the streamwise vorticity of a turbulent puff is mainly produced at the trailing edge by an inflectional instability, which results from the distortion of the parabolic velocity profile immediately upstream the sharp rear of the puff by the streamwise vortices. They developed both experimental and numerical methods to flatten the velocity profile at the upstream edge of the puff to intercept this mechanism and successfully relaminarised the puff. They also applied their experimental strategy to channel and duct flows and achieved relaminarisation of localized turbulence. Moreover, their control in numerical simulations was shown to work in fully turbulent flow at Reynolds numbers $R e \sim 3000$ if the force was imposed globally.

Based on the insights we gained into the emergence of fully turbulent pipe flow 
and the self-sustained mechanism of near wall turbulence, one of the goals of this thesis is to develop large scale control methods to relaminarise fully turbulent pipe flow by modifying the velocity profile and altering the flow structures.

\subsection{The outline}

This thesis mainly discusses two problems: the emergence of fully turbulent flow and turbulence control. In Chapter 2 the DNS codes used for this thesis will be introduced. A primitive-variable pipe flow DNS code based on projection and timesplitting schemes was developed and tested. In Chapter 3 the problem of the emergence of fully turbulent pipe flow will be discussed. The detailed work on the front speed measurement and spatial-temporal properties of turbulence fronts will be presented. Besides, the bifurcation scenario, together with Barkley's model, will be introduced. In Chapter 4, some turbulence control strategies that completely eliminate turbulence are presented. The relaminarisation of fully turbulent flow under partialslip boundary conditions is investigated in Chapter 5, where a literature review on superhydrophobicity will be given. 


\section{Chapter 2}

\section{Numerical METHOdS}

Turbulence is a multi-scale phenomenon. In wall shear turbulence, large scale structures extract energy from the wall shear and transfer the energy down to structures of smaller length scales until the energy gets dissipated by the structures at the viscous length scale. According to Kolmogorov's theory, the smallest length scale in turbulence, the so-called Kolmogorov length scale, $\eta \sim\left(\nu^{3} / \epsilon\right)^{1 / 4}$, where $\epsilon$ is the dissipation rate of the kinetic energy. Further, Taylor (1935) pointed out that $\epsilon \sim u^{3} / l$ where $u$ and $l$ are the characteristic velocity and length scales of the largest eddies, in pipe flow case $u \sim U$ and $l \sim D$. With these relations, the ratio of the kolmogorov length scale to that of the largest eddies, or the length scale of the width of the flow, will be $\eta / l \sim R e^{-3 / 4}$ (Tennekes \& Lumley, 1972). In three dimensional flow, this means a number of grid points $N \sim\left(R e^{3 / 4}\right)^{3}=R e^{9 / 4}$ if the smallest length scale should be resolved. At large Re, the kolmogorov length scale is orders of magnitude smaller than the geometry of the flow. Hence, to resolve turbulence at all length scales requires efficient high-resolution algorithms and a huge number of grid points. In engineering related $\mathrm{CFD}$, where the Reynolds number is usually high, it is unaffordable to resolve all scales directly. As a compromise, Reynolds-Averaged-Navier-Stokes equations (RANS) based approaches are usually adopted for these problems, in which the mean velocity and turbulent kinetic energy are solved, whereas the fluctuations are modeled with turbulence models that relate the Reynolds stress to the mean quantities. Another approach, the Large Eddy Simulation (LES), truncates the length cascades at a certain point. It solves the large energy containing scales and models smaller structures with sub-grid models (Moin \& Mahesh, 1998).

With the rapid development of computers and numerical methods, it became possible to resolve all length scales of turbulence at moderate Reynolds numbers by directly solving Navier-Stokes equations without introducing any turbulence models 
as RANS and LES do (Orszag \& Patterson, 1971). Nowadays, with high performance computers, direct numerical simulations (DNS) of Navier-Stokes equations have become a main tool in fundamental research of turbulence. In this thesis, DNS is performed with hybrid spectral-finite difference methods as explained in the following sections.

\subsection{DNS Code 1: a pressure-free formulation}

This code was developed by Ashley P. Willis (Willis \& Kerswell, 2009). It has been used in this thesis (Chapter 3 and Chapter 4) with minor modifications.

We consider the motion of incompressible fluid driven through a circular pipe with a fixed mass-flux. Normalizing length by the radius $R$ and velocity by $U_{\max }=2 U$, the Navier-Stokes equations read

$$
\frac{\partial \boldsymbol{u}}{\partial t}+\boldsymbol{u} \cdot \boldsymbol{\nabla} \boldsymbol{u}=-\nabla p+\frac{1}{R e} \Delta \boldsymbol{u}, \quad \boldsymbol{\nabla} \cdot \boldsymbol{u}=0
$$

where $\boldsymbol{u}$ is the velocity of the fluid and $p$ the pressure. These equations are solved in cylindrical coordinates $(r, \theta, z)$ using spectral-finite difference method with no-slip boundary conditions at the pipe wall $\boldsymbol{u}(1, \theta, z, t)=\mathbf{0}$ and periodicity in the axial direction. The pressure term is eliminated from the equations by using a toroidalpoloidal potential formulation of the velocity field (Willis \& Kerswell, 2009), in which the velocity is represented by toroidal $\psi$ and poloidal $\phi$ potentials, such that $\boldsymbol{u}=$ $\boldsymbol{\nabla} \times(\psi \hat{\boldsymbol{z}})+\boldsymbol{\nabla} \times \boldsymbol{\nabla} \times(\phi \hat{\boldsymbol{z}})$. After projecting the curl and double curl of the NavierStokes equations on the $z$-axis, a set of equations of the potentials $\psi$ and $\phi$ is obtained. A difficulty, due to the coupled boundary conditions on the potentials, is solved with an influence-matrix method. Assuming the periodicity in azimuthal and axial directions, the unknowns, i.e., the potentials, are expanded in Fourier modes,

$$
A(r, \theta, z, t)=\sum_{k=-K}^{K} \sum_{m=-M}^{M} \hat{A}_{k, m}(r, t) e^{(i \alpha k z+i m \theta)}
$$

where $\alpha k$ and $m$ give wave numbers of the modes in axial and azimuthal direction respectively, $2 \pi / \alpha$ gives the pipe length $L_{z}$, and $\hat{A}_{k, m}$ is the complex Fourier coefficient of mode $(k, m)$. With this expansion, the differential equations of the potentials $\psi$ and $\phi$ reduce to a set of linear systems for each $(k, m)$ mode, which are solved with a pseudo-spectral method. The nonlinear term is evaluated using the 
pseudo-spectral technique with the $\frac{3}{2}$-rule for the de-aliasing. In the radial direction spatial discretization is performed using finite difference method with a 9-point stencil. The time-dependent equations are integrated in time using a second-order predictor-corrector scheme with a dynamic timestep size, which is controlled using the information from a Crank-Nicolson corrector step. See (Willis \& Kerswell, 2009) For the details of the formulation and integration.

\subsection{DNS Code 2: a primitive-variable formulation}

Mainly for the work in Chap. 5, I developed a primitive-variable code in which the boundary condition can be easily modified. Here I adopt an efficient time-splitting pseudo-spectral algorithm for incompressible flow, a so called 'improved projection scheme' developed by Hugues \& Randriamampianina (1998).

\subsubsection{Formulation}

The primitive Navier-Stokes equations Eqs. (2.1) are solved in cylindrical coordinates $(r, \theta, z)$. Rewrite Eqs. (2.1) in the following form:

$$
\frac{\partial \boldsymbol{u}}{\partial t}+N(\boldsymbol{u})=-\nabla p+\frac{1}{R e} L(\boldsymbol{u}), \quad \nabla \cdot \boldsymbol{u}=0,
$$

and no-slip boundary condition is adopted (Neumann and hybrid conditions are also possible and easy to implement)

$$
\boldsymbol{u}=\boldsymbol{W} \quad \text { on } \partial \Omega
$$

where $\Omega$ is the flow domain and $\partial \Omega$ the boundary of $\Omega$, i.e., $r=1$ in pipe flow.

An important issue for incompressible Navier-Stokes solver is correctly treating the pressure term that couples with velocity field. To solve the pressure $p$, a Poisson equation for $p$ is derived from Eqs. (2.3) by taking the divergence on both sides:

$$
\Delta p=-\nabla \cdot N(\boldsymbol{u})
$$

note that linear terms vanish thanks to the incompressibility condition. A Neumann boundary condition can be derived by taking the derivative of Eqs. (2.3) with respect 
to the wall normal (radial) direction:

$$
\frac{\partial p}{\partial r}=\hat{\boldsymbol{r}} \cdot\left(-\frac{\partial \boldsymbol{u}}{\partial t}-N(\boldsymbol{u})+\frac{1}{R e} L(\boldsymbol{u})\right) \quad \text { at } r=1
$$

The spatial discretization is the same as in Code 1 . In radial direction we use finite-difference method for the radial derivative with a 9-point stencil for a lower computation cost compared to fully spectral algorithm. With the periodic boundary condition in axial and azimuthal directions, velocity is represented as Fourier expansion as shown in Eqs. (2.2). However, the pressure $p$ usually is not periodic in pipe flow case. Pipe flow is usually driven by a constant pressure gradient or by a constant mass flux with a variable pressure gradient. An overall axial pressure gradient, which is not periodic in the axial direction and can not be expanded as Fourier series, is present in either case. Throughout my thesis the pipe flow is driven by a fixed mass flux, which requires a time dependent axial pressure gradient. Denoting this pressure gradient as $P_{z}$, the pressure field finally takes the form:

$$
p(r, \theta, z, t)=\sum_{k=-K}^{K} \sum_{m=-M}^{M} \hat{p}_{k, m}(r, t) e^{(i \alpha k z+i m \theta)}+z P_{z}(t),
$$

where the first term on the right hand side is the periodic part and $z P_{z}$ the nonperiodic part of the pressure field.

\subsubsection{Integration}

The temporal integration is carried out with a second-order-accurate scheme based on a combination of backward differentiation schemes and Admas-Bashforth (the nonlinear term is treated with the Admas-Bashforth scheme). It reads:

$$
\frac{3 \boldsymbol{u}^{n+1}-4 \boldsymbol{u}^{n}+\boldsymbol{u}^{n-1}}{2 \Delta t}+2 N\left(\boldsymbol{u}^{n}\right)-N\left(\boldsymbol{u}^{n-1}\right)=-\nabla p^{n+1}+\frac{1}{R e} \Delta \boldsymbol{u}^{n+1}
$$

Given a proper initial condition $\boldsymbol{u}^{0}$ and set $\boldsymbol{u}^{-1}=\boldsymbol{u}^{0}$, the time-splitting steps proceed as following.

1. The predictor of the periodic part of a preliminary pressure field $\bar{p}^{n+1}$. The discrete form of the Poisson equation for pressure is:

$$
\Delta \bar{p}^{n+1}=\boldsymbol{\Delta} \cdot\left[-2 N\left(\boldsymbol{u}^{n}\right)+N\left(\boldsymbol{u}^{n-1}\right)\right]
$$


with Neumann boundary condition (discretized form of Eqs. (2.6) )

$$
\begin{aligned}
\frac{\partial \bar{p}^{n+1}}{\partial r}=\hat{\boldsymbol{r}} & \cdot\left\{\frac{-3 \boldsymbol{W}^{n+1}+4 \boldsymbol{u}^{n}-\boldsymbol{u}^{n-1}}{2 \Delta t}-2 N\left(\boldsymbol{u}^{n}\right)+N\left(\boldsymbol{u}^{n-1}\right)\right. \\
& \left.+\frac{1}{R e}\left[2 L\left(\boldsymbol{u}^{n}\right)-L\left(\boldsymbol{u}^{n-1}\right)\right]\right\} \quad \text { at } r=1 .
\end{aligned}
$$

Here the diffusion term is treated in the same way as the nonlinear term is. Furthermore, to improve the stability, the $L(\boldsymbol{u})$ is written in the following form (see Hugues \& Randriamampianina (1998)):

$$
L(\boldsymbol{u})=\Delta \boldsymbol{u}=\boldsymbol{\nabla}(\boldsymbol{\nabla} \cdot \boldsymbol{u})-\boldsymbol{\nabla} \times(\boldsymbol{\nabla} \times \boldsymbol{u})=-\nabla \times(\boldsymbol{\nabla} \times \boldsymbol{u})
$$

2. The predictor of a preliminary velocity field $\boldsymbol{u}^{*}$ with no-slip boundary condition Eqs. (2.4) and the preliminary pressure $\bar{p}^{n+1}$ solved in the above step:

$$
\frac{3 \boldsymbol{u}^{*}-4 \boldsymbol{u}^{n}+\boldsymbol{u}^{n-1}}{2 \Delta t}+2 N\left(\boldsymbol{u}^{n}\right)-N\left(\boldsymbol{u}^{n-1}\right)=-\nabla\left(\bar{p}^{n+1}+z P_{z}\right)+\frac{1}{R e} \Delta \boldsymbol{u}^{*}
$$

with

$$
\boldsymbol{u}^{*}=\boldsymbol{W}^{n+1} \quad \text { at } r=1
$$

In this step, the axial pressure gradient $P_{z}$ will be solved by imposing the constant mass flux condition:

$$
\int_{0}^{1} \int_{0}^{2 \pi} u_{z}^{*} r d r d \theta=M
$$

where $M$ is the constant mass flux. Since this pressure gradient $P_{z}$ is only time dependent, $\boldsymbol{\nabla}\left(z P_{z}\right)=P_{z} \hat{\boldsymbol{z}}$ only acts on the $(k=0, m=0)$ mode of the axial velocity component $u_{z}$. In addition, the constant mass flux condition Eqs. (2.14) also only concerns the $(k=0, m=0)$ mode of $u_{z}$ for the following reasons. Due to the Fourier representation of the velocity $\boldsymbol{u}^{*}$, the terms with azimuthal wave number $m \neq 0$ do not contribute to the mass flux since $\int_{0}^{2 \pi} e^{i m \theta} d \theta=0$ if $m \neq 0$. In addition, the mass flux on the pipe cross section should be axially invariant. Consequently, the terms with axial wave number $k \neq 0$ should neither contribute to the mass flux. Finally,

$$
\int_{0}^{1} \int_{0}^{2 \pi} u_{z}^{*} r d r d \theta=2 \pi \int_{0}^{1} u_{z, 00}^{*} r d r=M
$$

$u_{z, 00}$ can be solved combining Eqs. (2.12) and Eqs. (2.15). Other modes of $u_{z}$, 
and components $u_{r, k m}$ and $u_{\theta, k m}$ can be obtained by solving Eqs. (2.12) alone because $P_{z}$ is not directly involved in their dynamics.

3. Correct the predicted pressure $\bar{p}^{n+1}$ to enforce the incompressibility of the velocity field. Note that in the previous step, the condition $\boldsymbol{\nabla} \cdot \boldsymbol{u}^{*}$ is not imposed, hence, the predicted velocity field is generally not divergence free. In this step, the pressure filed $\bar{p}^{n+1}$ will be corrected to enforce the incompressibility. Consider the following equation

$$
\frac{3 \boldsymbol{u}^{n+1}-3 \boldsymbol{u}^{*}}{2 \Delta t}=-\nabla\left(p^{n+1}-\bar{p}^{n+1}\right) \quad \text { in } \Omega \text { and at } r=1
$$

and

$$
\boldsymbol{\nabla} \cdot \boldsymbol{u}^{n+1}=0 \quad \text { in } \Omega
$$

with boundary condition

$$
\hat{\boldsymbol{r}} \cdot \boldsymbol{u}^{n+1}=\hat{\boldsymbol{r}} \cdot \boldsymbol{u}^{*} \quad \text { at } r=1,
$$

Defining an intermediate variable $\phi=2 \Delta t /\left(3\left(p^{n+1}-\bar{p}^{n+1}\right)\right)$ and taking the divergence of Eqs. (2.16) with boundary condition Eqs. (2.18) result in a Poisson equation for $\phi$ :

$$
\Delta \phi=\nabla \cdot \boldsymbol{u}^{*} \quad \text { in } \Omega
$$

with a Neumann boundary condition

$$
\frac{\partial \phi}{\partial r}=0 \quad \text { at } r=1
$$

Eqs. (2.20) is derived from the boundary condition Eqs. (2.18). In fact, $\boldsymbol{u}^{n+1}-$ $\boldsymbol{u}^{*}=-\boldsymbol{\nabla} \phi$ according to the definition of $\phi$. Projecting this relation on the radial direction, one obtains

$$
\hat{\boldsymbol{r}} \cdot\left(\boldsymbol{u}^{n+1}-\boldsymbol{u}^{*}\right)=\frac{\partial \phi}{\partial r}=\hat{\boldsymbol{r}} \cdot\left(\boldsymbol{W}^{n+1}-\boldsymbol{W}^{n+1}\right)=0,
$$

note the boundary condition $\boldsymbol{u}^{*}=\boldsymbol{W}^{n+1}$ in the previous step. Once obtaining $\phi$, one can correct the pressure and velocity fields in $\Omega$ as well as on the wall by

$$
p^{n+1}=\bar{p}^{n+1}+\frac{3}{2 \Delta t} \phi, \quad \boldsymbol{u}^{n+1}=\boldsymbol{u}^{*}-\nabla \phi .
$$

In this corrector step, the mass flux does not change. In fact, the Poisson 
equation Eqs. (2.19) reduces to Laplace's equation $\Delta \phi=0$ for the $(k=0, m=$ 0) mode. Given the homogeneous Neumann boundary condition, the solution will be $\phi_{00}=$ const. in the whole domain. Clearly, this will not modify the $(k=0, m=0)$ mode of the velocity $u_{z}^{*}$ predicted in step 2 , which satisfies the fixed mass flux condition subject to Eqs. (2.15). In a word, $u_{z, 00}=u_{z, 00}^{*}$ and at the end of integration the velocity field satisfies both the constant mass flux and incompressibility condition.

In what follows, the laminar Hagen-Poiseuille flow, and the wall friction factor of fully turbulent flow will be tested.

\subsubsection{The Hagen-Poiseuille flow}

Given a pressure gradient, the Hagen-Poiseuille flow starts to develop. This test was carried out at $R e=4000$ with 64 Chebyshev points in the radial direction. The initial flow is one at still, i.e., $\boldsymbol{u}=0$ and the axial pressure gradient is set to be $-\frac{4}{R e}$. The analytic steady solution to the Navier-Stokes equations under these conditions is the parabolic Hagen-Poiseuille flow $\boldsymbol{u}=\left(1-r^{2}\right) \hat{\boldsymbol{z}}$, where $\hat{\boldsymbol{z}}$ is the unit vector in axial direction. Fig. 2-1(a) shows the evolution of the velocity profile in numerical simula-

(a)

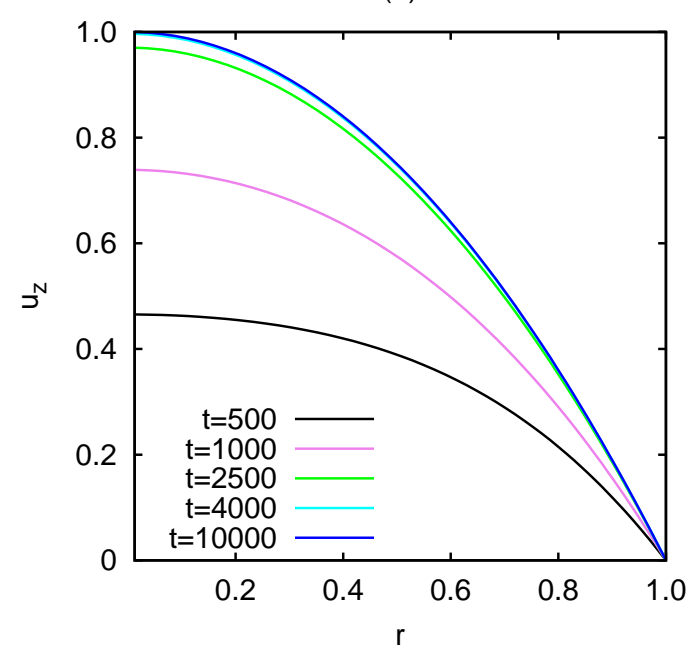

(b)

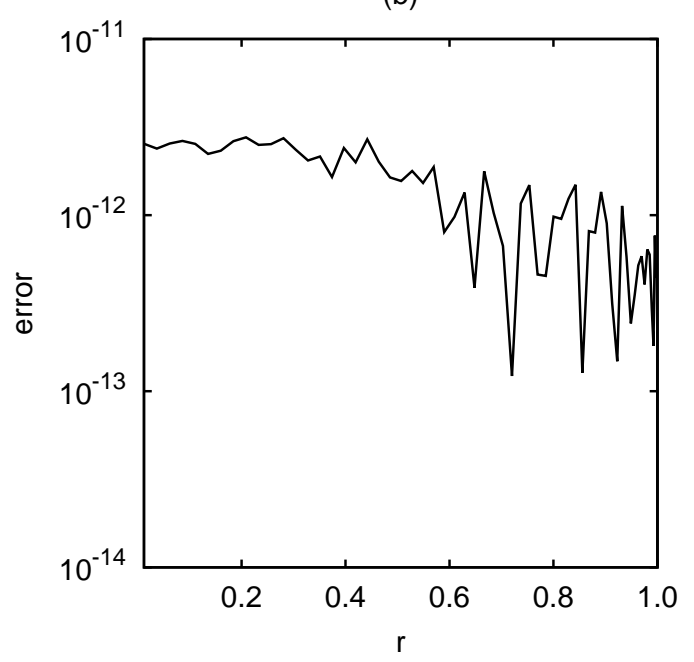

Figure 2-1: (a) The development of the velocity profile under pressure gradient $\frac{\partial p}{\partial z}=$ $-\frac{4}{R e}$ starting from $\boldsymbol{u}=0$. (b) The error of the converged numerical solution.

tion. The velocity profile at $t=4000$ (the cyan line which overlaps with the blue line at $t=10000$ ) is almost the parabolic flow (with an error below \%0.5). Fig. 2-1)(a) shows the error of the converged solution. With this radial resolution the error is on 
the order of magnitude of $10^{-12}$.

A second test is to fix the flow rate at $\int_{0}^{2 \pi} \int_{0}^{1} u_{z} r d r d \theta=\frac{\pi}{2}$ and solve the corresponding driving pressure gradient. The resolution and $R e$ are the same as the previous test. The code gives accurately $1 \times 10^{-3}=4 / R e$ (relative error below $10^{-12}$ ). These two tests show that this code can accurately produce the Hagen-Poiseuille flow.

\subsubsection{Wall friction of fully turbulent flow}

The wall friction factor is calculated for fully turbulent flow at several Reynolds numbers so to test the code. Simulations are performed at $R e=3000,5000,7500$, 10000, 15000, and 20000. For $R e=3000$ and 5000 a pipe with $L_{z}=8 \pi$ is used, and at other higher Reynolds numbers a pipe with $L_{z}=4 \pi$ is used considering the high computation cost. The domain size, resolutions, viscous Reynolds number $R e_{\tau}$, and comparison of DNS $C_{f}$ and that given by Blasius law $C_{f}=0.3164 R e^{-0.25}$ are listed in Tab. 4.1. Clearly, this code can accurately capture the friction factor and the agreement with the Blasius law is very good (within an error of $1.6 \%$ at all these Reynolds numbers).

\begin{tabular}{llllllll}
\hline$R e$ & $L_{z}$ & $N$ & $M$ & $K$ & $R e_{\tau}=\frac{u_{\tau} R}{\nu}$ & $C_{f}$ & $C_{f, \text { Blasius }}$ \\
\hline 3000 & $8 \pi$ & 64 & 40 & 192 & 109.35 & 0.0423 & 0.0427 \\
5000 & $8 \pi$ & 80 & 60 & 256 & 172.77 & 0.0382 & 0.0376 \\
7500 & $4 \pi$ & 128 & 128 & 128 & 245.01 & 0.0342 & 0.0340 \\
10000 & $4 \pi$ & 144 & 144 & 144 & 315.20 & 0.0318 & 0.0316 \\
15000 & $4 \pi$ & 160 & 160 & 160 & 448.20 & 0.0286 & 0.0286 \\
20000 & $4 \pi$ & 192 & 192 & 160 & 580.69 & 0.0270 & 0.0266 \\
\hline
\end{tabular}

Table 2.1: The domain size $L_{z}$, resolutions $(N, M, K)$ with $N$ the number of Chebyshev point in radial direction, the viscous Reynolds number $R e_{\tau}$ based on timeaveraged friction velocity $u_{\tau}$ at the wall, the time-averaged $C_{f}$ and $C_{f}$ given by Blasius law. Note that the actual resolution in spectral space is $N \times 2 M \times 2 K$ with $N \times 3 M \times 3 K$ grid points in physical space because of the $\frac{3}{2}$-dealiasing. The domain size in wall unit is $L^{+}=L_{z} R e_{\tau}$. 


\section{CHAPTER 3}

\section{THE EMERGENCE OF FULLY TURBULENT FLOW}

After a century of extensive studies, the origin of fully turbulent flow in pipes has not yet been resolved. Research in recent decades showed the intermittent nature associated with the localized turbulence, puffs, that appear at $R e \sim 2000$ (Wvgnanski \& Champagne, 1973; Wvgnanski et al., 1975; Darbvshire \& Mullin, 1995; Faisst \& Eckhardt, 2004; Hof et al., 2006; Peixinho \& Mullin, 2006; Willis \& Kerswell, 2007; Hof et al., 2008; Avila et al., 2010). It has been speculated (Moxey \& Barkley, 2010) and clarified (Avila et al., 2011) that localized turbulence becomes sustained via spatiotemporal intermittency. The competition between puff decay and splitting determines that the onset of sustained turbulence is at $R e=2040$ (Avila et al., 2011). However, at the onset individual turbulence patches still stay localized keeping a constant axial extension. At higher Reynolds numbers above $R e \sim 2800$, studies showed that turbulence grows continuously as slugs and pipe flow eventually becomes fully turbulent. Puffs and slugs are bounded by upstream and downstream fronts, and the development of turbulence can be depicted by the speed of these fronts which has been the subject of extensive studies (Lindgren, 1969; Wvgnanski \& Champagne, 1973; Sreenivasan \& Ramshankar,, 1986; (Nishi et al., 2008; Duguet et al., 2010b). The speed data of these studies roughly agree and collectively showed a smooth change from puffs to slugs. However, so far no clear scenario of transition to fully turbulent flow has been obtained and no bifurcation point has been determined. In order to better understand this transition process, in this chapter, I first show accurate front speed data from extensive DNS measurement. Then a new pipe flow model by Barkley, a descendant of the the earlier models of Barkley $(2011 b, \underline{a})$, will be in- 
troduced and the results from theoretical modeling will be compared with my DNS results and experimental results from Mukund Vasudevan at IST Austria. At the end, combining all these approaches, the transition scenario to fully turbulent flow will be explained.

\subsection{Speed measurement}

\subsubsection{Detection of the fronts and thresholding}

In the following I will discuss the procedure developed to determine the speed of the turbulence fronts. To study the front speed, the laminar flow was locally perturbed and the evolution of the resulting turbulent patch was studied. As initial perturbations, we selected velocity fields from localized turbulent structures (i.e., puffs) at lower $R e, R e=1950$ and 2000. Here puffs remain in equilibrium for very long times. They keep a constant size and travel downstream at an almost constant speed. At the same time they are intrinsically chaotic and memoryless (Faisst \& Eckhardt, 2004; Song \& Hof, 2014). This makes them ideally suited as initial localized perturbations for statistical studies at higer $R e$. To make sure initial conditions are not strongly correlated with each other, consecutive ones are separated by approximately 80 time unit. To detect the turbulent fronts, here the quantity $q(z):=\iint\left(u_{r}^{2}+u_{\theta}^{2}\right) r d r d \theta$ is chosen as the local turbulence intensity which is zero in laminar regions and of a finite value in turbulent regions. By setting a proper threshold above which flow will be considered as turbulent, the downstream and upstream fronts can be easily detected. Other quantities such as centerline velocity and streamwise vorticity $\omega_{z}$ could be equally well used.

To illustrate the procedure of locating the fronts, an example is shown in Fig. 3-1. It shows a puff at $R e=2000$ in a pipe with a length $L_{z}=266$, which is characterized by a sharp upstream front and a more diffusive downstream front. Fig. 3-11(a) shows the distribution of the local turbulence intensity $q$ along the pipe, and Fig. 3-1(b) shows the distribution of the centerline velocity. As mentioned above, in principle both of them can be chosen for locating the fronts. In Fig. 3-1(a) three dashed lines are plotted corresponding to three different thresholds $5 \times 10^{-4}, 10^{-5}$ and $10^{-6}$. Obviously they give different lengths of the structure, $5 \times 10^{-4}$ gives a length about 18 , $10^{-5}$ gives about 40 , and $10^{-6}$ about 65 . Because of the periodic boundary condition imposed in the axial direction, structures can be followed for long times. On the other hand, the downstream and upstream fronts are eventually going to meet for 
(a)

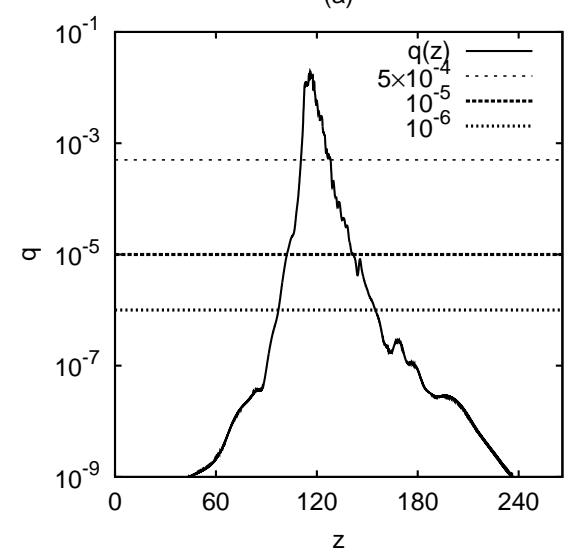

(b)

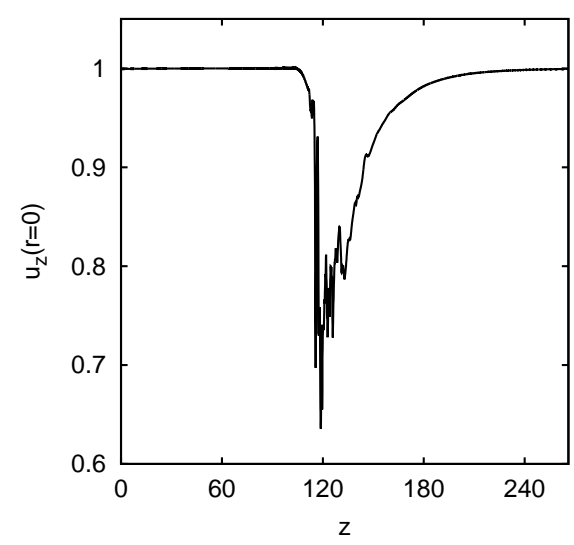

Figure 3-1: (a) The distribution of the local turbulence intensity $q$ as a function of axial position $z$ at $R e=2000$. The data are taken at a random time instant in a pipe with $L_{z}=266$. The three dashed lines show three possible thresholds for locating the downstream and upstream fronts. Other proper values are also eligible. (b) The streamwise velocity on the pipe axis taken at the same time as (a) is.

slugs. Therefore, the measurement has to stop when these two fronts get too close to each other because the interaction between them may affect their intrinsic speeds. In run time, the $q(z)$ will be calculated frequently in order to give a detailed time series of the locations of fronts, based on which front speed will be calculated. In the following, the position and the speed of fronts will be determined with these three thresholds, and the influence of the thresholding on the front speed will be studied.

Front positions and speeds resulting from the three different thresholds as plotted as dashed line in Fig. 3-1 (a), are compared in Fig. 3-2, The position is plotted in a frame of reference co-moving with the mean flow (see Fig. 3-2(a)). By doing so, the speed of the fronts relative to the mean flow can be seen straightforward from the figure. Apparently, three thresholds result in different positions of the fronts as well as different lengths of the puff. However, it is quite obvious that in Fig. 3-2(a), the lines for either the upstream or downstream front are overall parallel to each other, indicating that different thresholds indeed give the same overall speed of the fronts. As known, puffs are usually characterized by a sharp upstream edge and a relatively more diffusive downstream edge, which is also reflected in this figure. The three dotted lines for the upstream front are smoother than those three for the downstream front, regardless of the threshold. The fluctuations of positions are due to the fact that at the fronts, turbulent eddies keep shedding off and escape from the main structure, or disconnected turbulent islands even merge into to the main structure. This fluctuation is also partially a consequence of the simple treatment of the fronts 
(a)

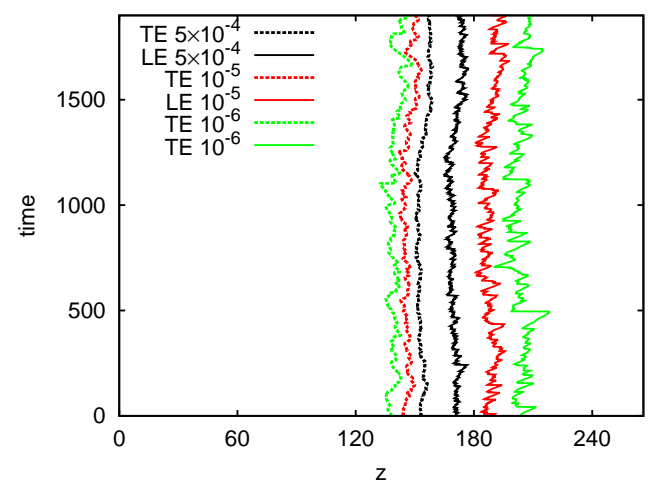

(b)

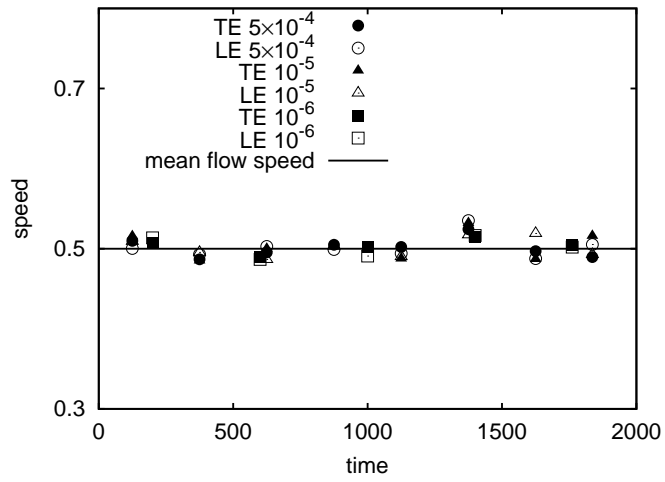

Figure 3-2: (a) The streamwise propagation of the puff shown in Fig. 3-1 in a frame comoving with the mean flow (at a speed of 0.5 ). The axial position of the downstream (LE, as solid line on downstream) and upstream (TE, as dashed line on upstream) fronts are detected based on three thresholds, $5 \times 10^{-4}, 10^{-5}$, and $10^{-6}$ (as shown in Fig. 3-1(a) as dotted lines), which are separated from each other by an oder of magnitude or even more. (b) The average speed of the fronts detected with all three thresholds in some time intervals based on the information in (a)

as a threshold. As a matter of fact, a simple cut-off clearly can not capture the full features of the highly convoluted laminar-turbulent interfaces (Holzner et al., 2013). The other piece of information from this figure is that the puff propagates downstream at a speed very close to the mean flow speed. From the beginning to about $t=1200$, and after about $t=1500$ the positions of both fronts are rather constant in the comoving frame. However, between $t=1200$ and 1500 the puff accelerates a bit and moves downstream slightly faster than the mean flow. Then it decelerates and regains the mean speed of the flow. This can be more quantitatively demonstrated by the time series of the front speed, as shown in Fig. 3-2(b). Considering the fluctuations in the front position, the time window in which the speed is averaged should be large compared to the characteristic time scale of the fluctuations. In this figure, for thresholds $5 \times 10^{-4}$ and $10^{-5}$ the speed is averaged over 250 time unit, and for threshold $10^{-6}$ a larger time interval 400 is adopted to avoid the front speed being affected by the bigger fluctuations in the downstream front, as shown by green lines in Fig. 3-2(a). Fig. 3-2(b) indicates that the speeds measured with different thresholds agree well with each other. The most straightforward estimation of the overall mean speed of the fronts is $\frac{z_{\text {end }}-z_{0}}{t_{\text {end }}-t_{0}}$. In this estimation, the threshold $5 \times 10^{-4}$ gives 0.502 and 0.502 for the upstream and downstream fronts respectively, $10^{-5}$ gives 0.503 and 0.502 , and $10^{-6}$ gives 0.502 and 0.503 . All of them give very close overall mean speed of the fronts. Here it is concluded that the specific threshold does not considerably 
affect the result of the front speed. In what follows, $5 \times 10^{-4}$ is chosen as the threshold at all Reynolds numbers for the sake of comparison.

It should be noted that in the splitting regime and the early slug regime (up to about $R e \simeq 2800$ ), only the most upstream and the most downstream fronts will be detected, ignoring laminar gaps or low intensity holes that may appear inside the structures(Avila \& Hof, 2013).

Here, pipe flow is treated as a quasi one-dimensional flow (as $q(z)$ ) because the front dynamics of pipe flow are indeed rather one-dimensional and can be very well modelled by one-dimensional models (Barkley, 2011b, a ). In fact, it is possible to treat the actual 3-dimensional fronts and calculate the local propagation speed of the fronts. Holzner et al. (2013) developed a quantitative Lagrangian approach to calculate the local propagation speed of turbulence. By defining the laminar-turbulent fronts (interfaces) as isosurfaces of the local enstrophy, the fronts, especially the downstream front, were found to be highly convoluted and stretched in the streamwise direction by the mean shear. The local propagation speed of the fronts was calculated and found to be distributed differently on the upstream and downstream front. Besides, the contributions from different mechanisms (e.g. diffusion and linear advection) to the propagation speed can be investigated with this method. Unfortunately, it requires extremely high resolution for the isosurfaces construction and interpolation from grid points onto isosurfaces, which makes it unaffordable for studies at higher Re and in long pipes (for details see Holzner et al. (2013)). Nevertheless, the overall propagation speed of the fronts of puffs at $R e=2250$ was evaluated as the speed of the mass center of the complex 3 -dimensional fronts, which is 0.47 and turned out to agree with the DNS result here as will be shown in the following.

\subsubsection{Temporal behavior of the front speed, $t_{0}$ and $L_{0}$}

In this section I discuss the temporal evolution and some statistics of the front speed of puffs and slugs. Tab4.1 lists the domain size and resolution at all Reynolds numbers considered in this study.

Fig. 3-3 shows the time series of the speed of the two fronts at Reynolds numbers 2200, 2400, 3000, and 5500. At each Reynolds number, the time series of the front speed from 6 runs are plotted and at any data point the speed is averaged over 80 time units. Overall it shows that the speed of the upstream front undergoes less fluctuations than that of the downstream front even at $R e$ as high as 5500, indicating that the upstream front is always energetically more stable than the downstream front. 
Chapter 3. The emergence of fully turbulent flow

\begin{tabular}{lllll|lllll}
\hline$R e$ & $L_{z}$ & $N$ & $K$ & $M$ & $R e$ & $L_{z}$ & $N$ & $K$ & $M$ \\
\hline 1910 & $48 \pi$ & 48 & 640 & 32 & 3000 & 360 & 72 & 2560 & 48 \\
1920 & $48 \pi$ & 48 & 640 & 32 & 3200 & 360 & 72 & 2560 & 54 \\
2000 & $48 \pi$ & 48 & 768 & 40 & 3500 & 360 & 72 & 2560 & 54 \\
2200 & $48 \pi$ & 48 & 768 & 40 & 3750 & 266 & 72 & 2048 & 54 \\
2300 & 266 & 64 & 1536 & 40 & 4000 & 266 & 72 & 2048 & 54 \\
2400 & 266 & 64 & 2048 & 48 & 4250 & 360 & 80 & 3072 & 60 \\
2500 & 266 & 64 & 2048 & 48 & 4500 & 360 & 80 & 3072 & 64 \\
2600 & 266 & 64 & 2048 & 48 & 5000 & 360 & 80 & 3072 & 64 \\
2800 & 360 & 72 & 2560 & 48 & 5500 & 360 & 96 & 3840 & 80 \\
\hline
\end{tabular}

Table 3.1: The domain size and resolution for the simulation. Note that in physical space there are $3 K$ and $3 M$ grid points in axial and azimuthal directions.

Interestingly the downstream front of puffs at $R e=2200$ seems to be more stable than that at $R e=2400$ and even up to 3000. It implies that slugs at low Reynolds numbers (below 3000) have a puff-like downstream front which is even more erratic and less well-defined than that of puffs. In fact, these slugs were referred to as puffs out of equilibrium or puffs that grow (Wygnanski \& Champagne, 1973; Duguet et al., $2010 b)$. Later we will clarify that they are actually slugs and are significantly different from puffs. It is worth mentioning that, at $R e \simeq 2400$, the downstream front manifests huge fluctuations and may even temporarily almost go backward relative to the laboratory frame due to fast decay (destruction) of the structures at the downstream edge. As pointed out earlier, this is partially a consequence of the simple treatment of the front as a cutoff, nevertheless, the huge fluctuations indicate the erratic nature of the downstream front in this regime.

Statistics were performed for a clearer trend of the front speed in time. The speed was first measured in individual runs, then the speed was averaged over all runs (about 20 for each $R e$ ) and finally a time series of the sample-averaged speed was obtained. In each individual run, the speed at any time instant was averaged within a time window of 80 time units. The length of the structure was concurrently measured for further analysis. Fig. 3-4 shows the temporal evolution of the sample-averaged front speed at $R e=2200,2400,3000,3750,4250$, and 5000, from the puff regime to slug regime. As shown in (a-c), at low Re the front speed does not show much transients on average after the initiation. At $R e=2200$ this is expected since initial conditions are puffs nearby at $R e=2000$. However, it could be that at $R e=2400$ and 3000 as shown in (b) and (c), the fluctuations of the erratic downstream front mask the initial transients (see also Fig. 3-3). At higher Re, on the contrary, the front speed, particularly that of the downstream front increases after the initiation, 

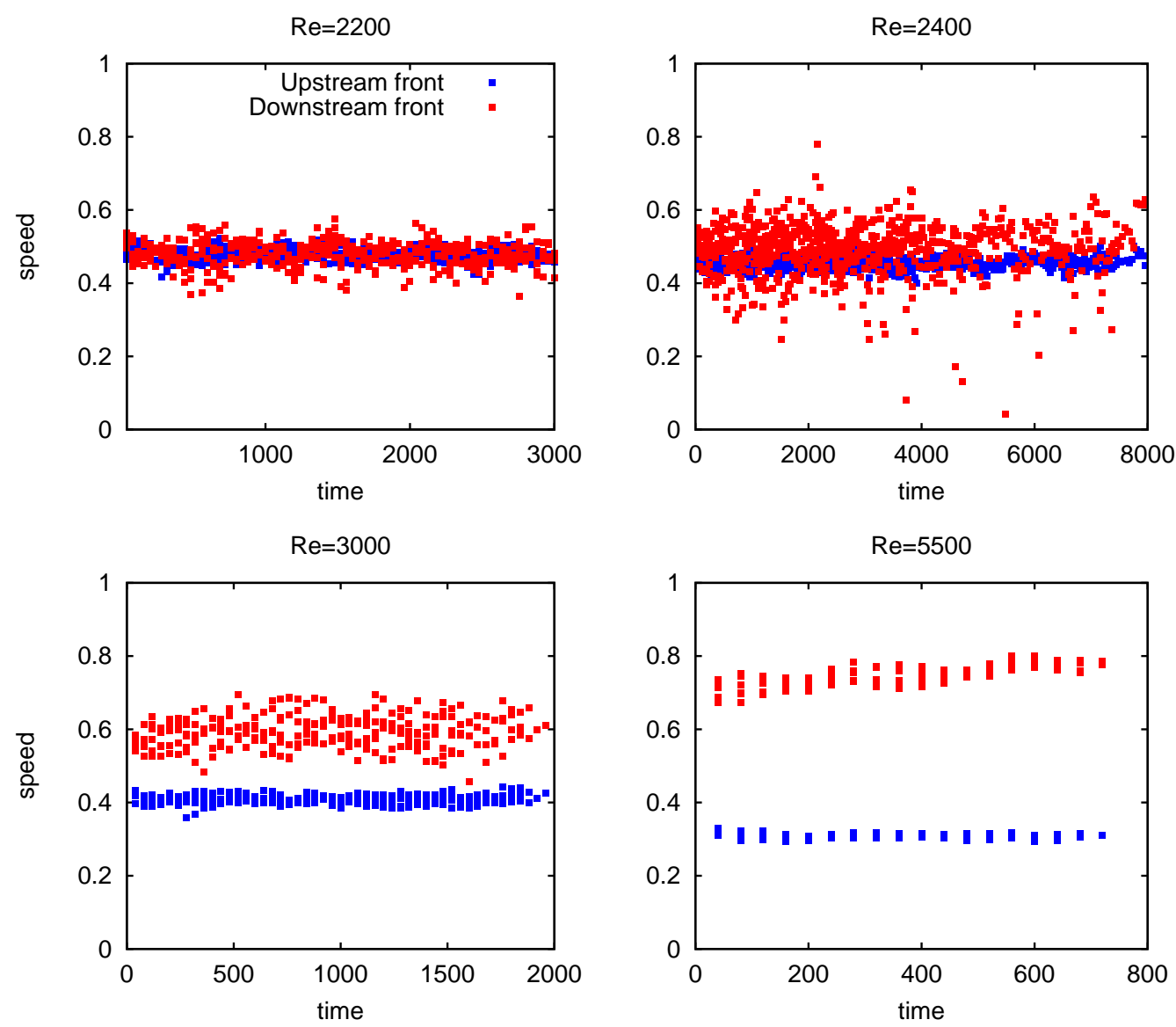

Figure 3-3: Time series of the front speed at Reynolds numbers 2200, 2400, 3000, and 5500. The red points represent downstream front and the blue ones upstream front. At any time instant, speed is averaged over 80 time units with the time instant in the middle of the averaging window. Only 6 runs are plotted at each Reynolds number to avoid overcrowding.

and saturates after a certain time. This duration from initiation to speed saturation is referred to as $t_{0}$, i.e., the structure formation time. As can be seen, at $R e=3750$ the speed saturates at about $t=300,400$ at $R e=5000$, and about 500 at $R e=5500$. This trend indicates that it takes longer for slugs to reach a stable growth rate as $R e$ increases. If the speed should be measured, this $t_{0}$ has to be considered and excluded. Nishi et al. (2008) (figure 14) also observed the drastic velocity increase in the initial transient stage up to time 600 at $R e>4530$, and the higher the Reynolds number, the longer the initial transients. Above $R e=9000$, the downstream front speed has not even stabilized within their $L_{z}=1066$ pipe. However, in their experiment, this initial transients is partially due to the development of the Hagen-Poiseuille flow since the pipe inlet. In the DNS simulation on the other hand, the basic flow is already parabolic from the very beginning. Therefore, the $t_{0}$ in these two studies are not di- 
rectly comparable. My DNS results suggest that even starting with a fully developed parabolic flow, measurement should start at least 400 time units after the initiation below $R e=5000$, and at least 600 time units at $R e=5500$.

The relationship between front speed and the structure length was also studied. The results are shown in Fig. 3-5. Since puffs do not grow in time, they are not considered here. As shown in the figure, the relationship between the front speed and structure length behaves similar to that between the front speed and time. At low Re, for example at 2400, on average there is not significant change from the initiation. As mentioned before, the intrinsic large fluctuations of the downstream front speed may have masked the initial transients. However, from $R e=3000$ on, a clear increasing trend in the downstream speed can be observed during the initial transients. The speed saturates after the structure has picked up a certain length, which is referred to as $L_{0}$, i.e., the formation length of slugs. At $R e=3000, L_{0} \sim 60$ and as $R e$ increases $L_{0}$ also increases significantly. At $R e=5000$ and $5500, L_{0}$ gets as large as about 200 and 220. In order to have a sufficiently long measurement duration, the flow domain must be considerably longer than $L_{0}$. These results may provide both numerical and experimental measurements with a criterion for avoiding the effects of system size and initial transients.

Overall, the upstream front is rather stable since the initiation at any Reynolds number and is not even affected (at least not noticeable in this study) by the puff/slug formation period, however, Nishi et al. (2008) observed transients for both fronts. Besides the development of the Hagen-Poiseuille flow in their experiments, another possible reason for this is that all simulations were initiated with puffs simulated at $R e=2000$, which already have well-defined sharp upstream fronts. Nevertheless, the $t_{0}$ and $L_{0}$ obtained from the analysis on the downstream front should be sufficient for getting rid of the initial transients, as the upstream front is expected to form and stabilize faster than the downstream front if initiated with featureless perturbations (see figure 14 in Nishi et al. (2008)).

\subsubsection{Speed PDFs}

The front speed manifests different levels of fluctuations at different Reynolds numbers (see Fig. 3-3), which can be best depicted by the probability density function (PDF) of the speed. As shown in Sec. 3.1.2, the $t_{0}$ and $L_{0}$ have to be taken into consideration for accurate measurements. The speed was measured after $t_{0}=400$ for puffs, which do not grow, and after $L_{0}=80$ for slugs at $R e \leq 3000,120$ at $3000<R e \leq 4000$, 

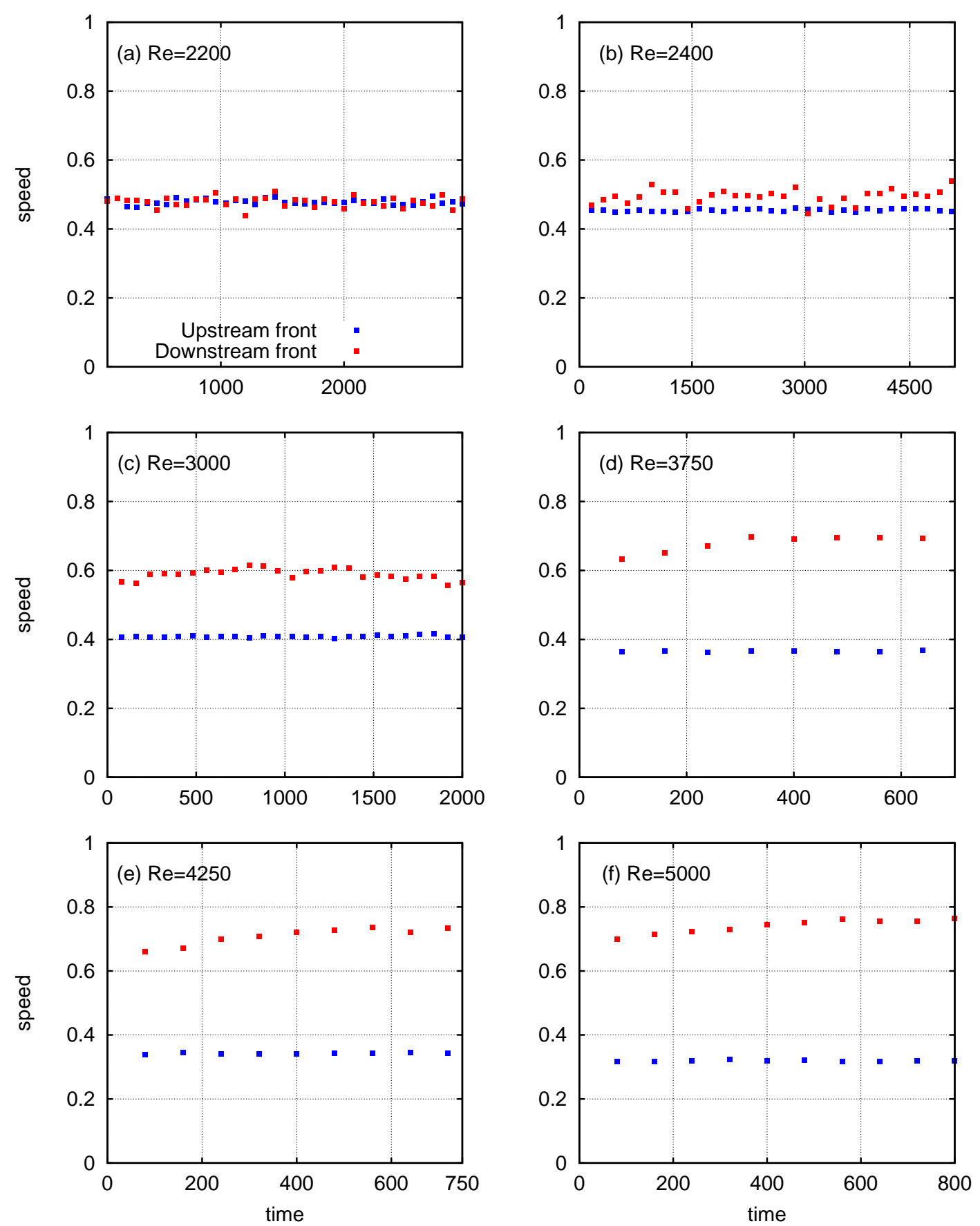

Figure 3-4: Time series of the averaged front speed at $R e=2200,2400,3000,3750$, 4250, and 5000. Each data point is an average over about 20 runs at the same time instant. While in each individual run, the speed at any time instant is averaged over 80 time units. 

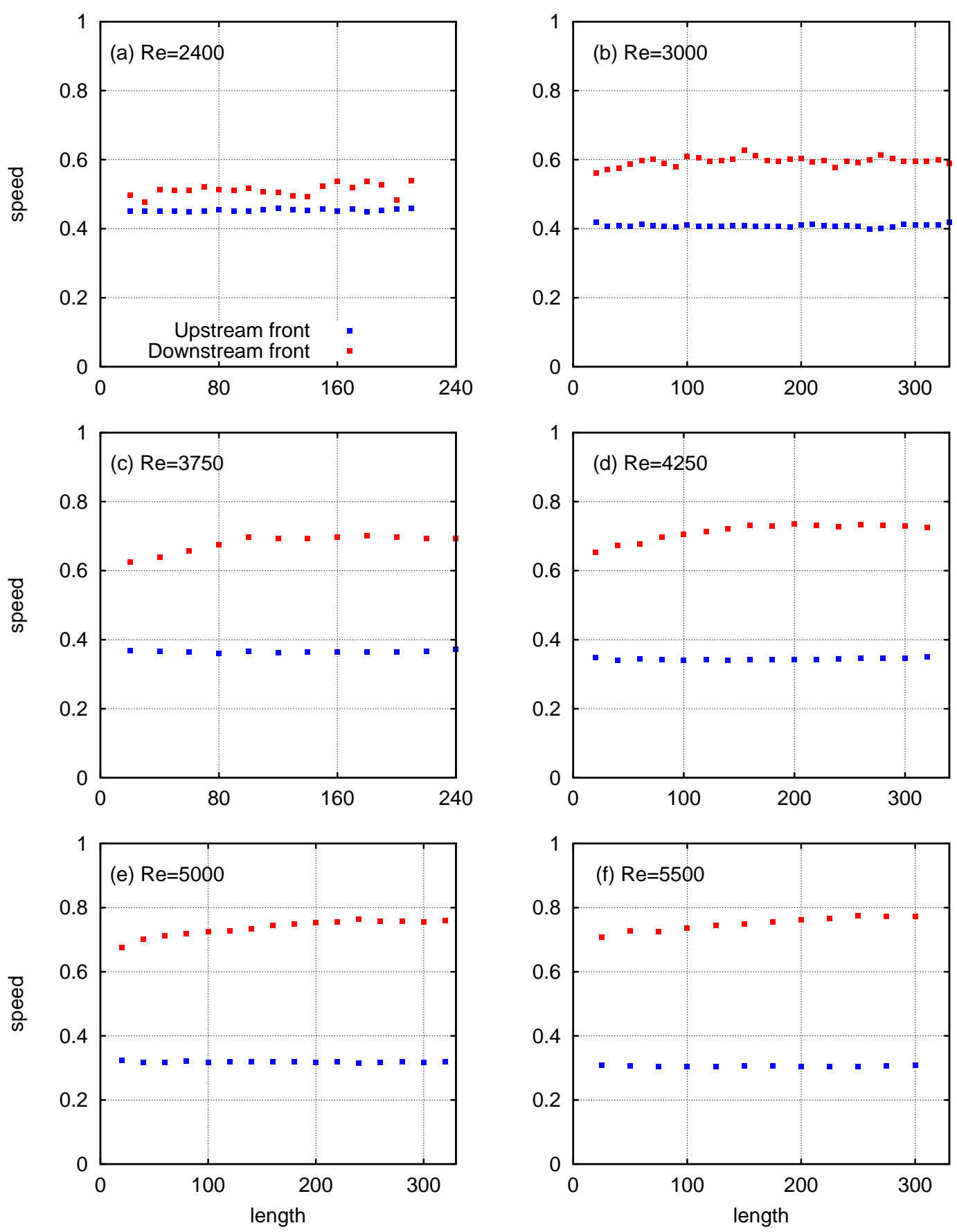

Figure 3-5: Time series of the averaged front speed at $R e=2400,3000,3750,4250$, 5000, and 5500. Each data point is an average over about 20 runs at the same length instant. 
160 at $4000<R e<5000$, and 240 at $R e \geq 5000$. The results are shown in Fig. 3-6.

The PDFs of the upstream front are similar at all Reynolds numbers, showing a sharp peak (actually slim bell-shaped distribution) symmetric to the mean. As Reynolds number increases, the PDF becomes slimmer and taller, indicating that the speed undergoes smaller fluctuations relative to the mean. This is consistent with the time series of upstream front speed shown in Fig. 3-3. However, above $R e=3750$ the PDF seems to have saturated. On the other hand, the PDFs of the downstream front speed are much fatter and shorter than that of the upstream front speed, indicating larger fluctuations in speed. The PDF is the fattest at about $R e=2400$ because

(a) Upstream front

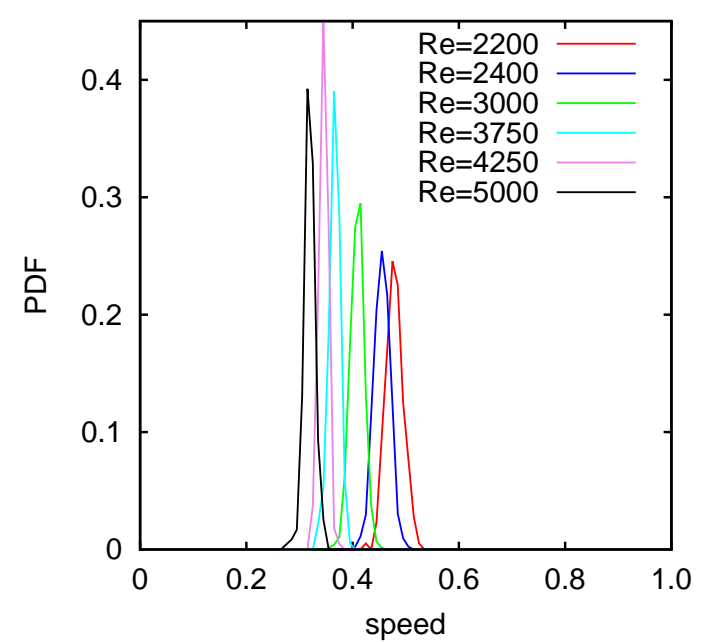

(b) Downstream front

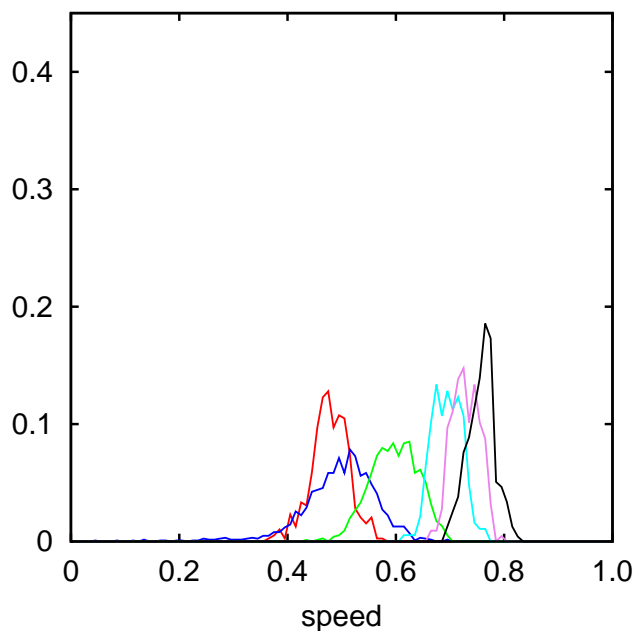

Figure 3-6: PDF of the front speed of puffs/slugs at $R e=2200,2400,3000,3750$, 4250, 5000. The same binning is used for all Reynolds numbers and the ruggedness in PDFs of the downstream front speed is due to the small sample size.

of the erratic downstream front resulting from the complicated immature slugs, and puff-puff/puff-slug interactions. Above $R e=2800$ slugs tend to become mature and grow continuously. Consistent with the information conveyed in Fig. 3-3, the PDF of the puff is slimmer than that of slugs below $R e \simeq 3000$ and is similar to that of slugs at $R e \simeq 3000$, suggesting that the downstream front of puffs is surprisingly more stable than that of early slugs, at least in the respect of front speed. As Reynolds number increases further, the PDf becomes slimmer and closer to that of the upstream front speed. The downstream front becomes more stable and undergoes less fluctuations when Reynolds number increases, implying that at high Reynolds numbers (above $R e \simeq 5000)$ slugs possess a downstream front similar to the upstream front, which will be discussed in more detail later in this chapter. 


\subsubsection{The front speed as a function of $R e$}

Because of the fluctuations of the fronts, especially the downstream front, sufficient observation time are needed to obtain an accurate mean speed. In this study, pipe lengths of 266 and 360 are adopted for simulations above Reynolds number 2300 and $48 \pi$ for puffs below 2200 (note that puffs do not grow). The details of the domain size and resolutions are shown in Tab. 4.1.

For each Reynolds number, data from about 20 runs were collected. In the puff regime, for each run, the speed measurements started after the puff had evolved about 400 time units in order to get rid of the initial transients. In the slug regime, measurements was started after the structure grew above 160 radii with the threshold $5 \times 10^{-4}$. In $L_{z}=266$ pipe the measurements stopped when the structure length reached 240, and 320 in $L_{z}=360$ pipe so to avoid the interactions between the upstream and downstream fronts when they get too close (Note the periodic boundary condition used in DNS and eventually the two fronts will meet each other and the pipe will be completely filled with turbulence). More details on the spatial properties of slugs are about to be discussed in the next section. The speed was first averaged in each run and denoted as $\bar{v}_{i}$, and further averaged over all runs and is denoted as $<\bar{v}_{i}>=\sum_{i} \bar{v}_{i} / N$. Because the initial conditions are uncorrelated, it is assumed that the speed measured in each run is independent of that in others. Besides, the mean speed of a single run is considered as a random variable and speeds of all runs are subject to an identical probability distribution. Under these assumptions, when $N$, the number of runs, is large, the population mean speed can be approximated by the sample mean $\left\langle\bar{v}_{i}>\right.$ and $1.96 \sigma / \sqrt{N}$ gives a $\% 95$ confidence interval for the mean, where $\sigma$ is the standard deviation of the sample $\bar{v}_{i}$. This confidence interval is taken as the uncertainty of our statistics as shown in Fig. 3-7.

Fig. 3-7 plots the front speed against the Reynolds number. Up to $R e=2200$, the speed of the two fronts are identical, resulting in a fixed length for puffs. From $R e \simeq 2300$ on, the speed of the two fronts starts to depart considerably. This overall agrees with former measurements (Lindgren, 1969; Wygnanski \& Champagne, 1973; Durst \& Ünsal, 2006; Nishi et al., 2008). As a consequence, localized turbulence starts to grow at a noticeable rate. This indicates that the onset of spreading turbulence is close to $R e \simeq 2300$. Unfortunately, in this regime, the turbulence spreading process occurs on very large time scales (Avila \& Hof, 2013), which makes numerical measurement very expensive. The speed from only a single run (without statistics) at $R e=2300$ is shown in Fig. 3-7. Small changes in the curvature could be seen for 


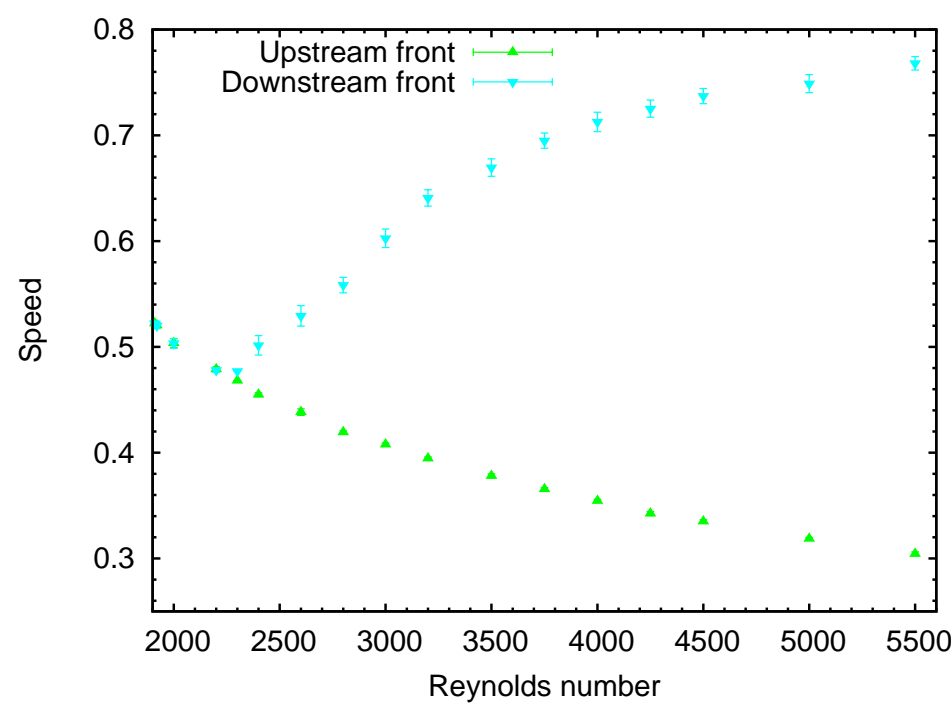

Figure 3-7: The front speed as a function of Reynolds number. For each point, about 20 runs are carried out and the error bars are uncertainties which give a $95 \%$ confidence interval of the mean speed as explained in the main text.

above $R e>2300$. This point will be stressed later.

The growth rate of turbulence as a function of Reynolds number is also investigated (see Fig. 3-8). The growth rate is given simply by the difference in the speed of the two fronts. The data show that the growth rate above the onset of the growth of turbulence $(R e \sim 2300)$ increases approximately linearly until $R e \sim 3200$. A linear fit (the black line in Fig. (3-8) very well represents the trend of the growth rate near the onset of growing turbulence. This disagrees with the results reported by Sreenivasan \& Ramshankar (1986); de Lozar \& Hof (2010), where the authors claimed a square root scaling between the growth rate and the distance to the 'critical' Reynolds number where the growth of turbulence first occurs. If taking the growth rate as the order parameter, this linear scaling suggests that this transition process does not fall in any universality class of non-equilibrium phase transition as reviewed by Hinrichsen (2010).

To my knowledge this is the first extensive DNS measurements of the front speed of puffs/slugs and the DNS data overall agree with former experimental measurements. In addition, the DNS speed data show much less scattering than those reported and give a nice estimation of the uncertainty of the statistics. However, the speed measurement alone does not suffice to explain the transition to fully turbulent flow. Besides, at $R e \simeq 2300$, puff splitting contributes to the difference between the speed of the two fronts, but structures still remain localized. Consequently, puff splitting does not signal the emergence of slugs. Actually there is no clear division between puffs and 


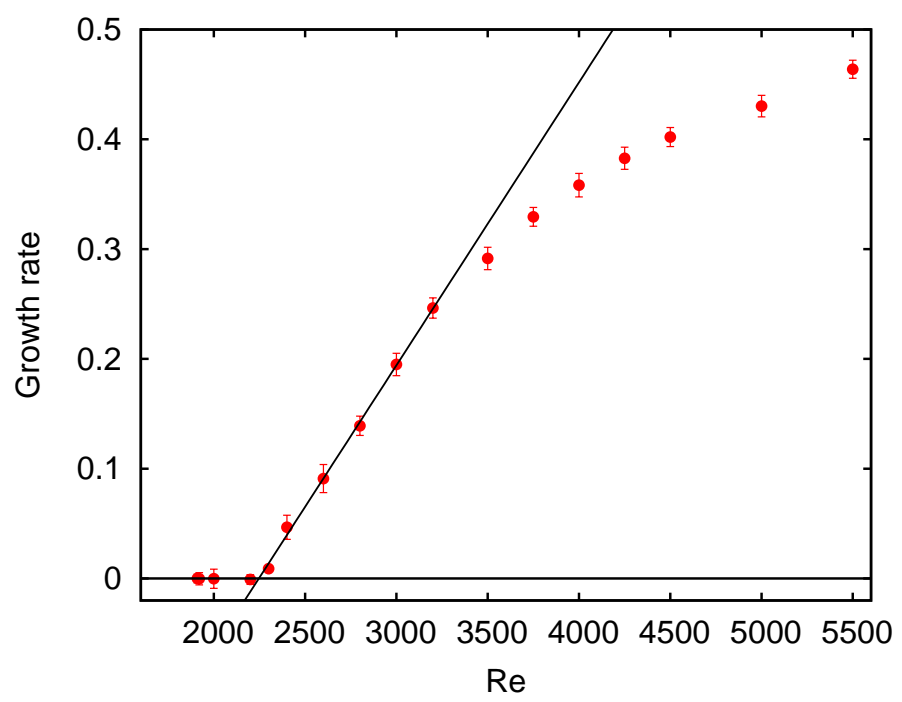

Figure 3-8: The growth rate of puffs/slugs. The black line is a linear fit with the data at $R e \in(23003200)$.

slugs between $R e \simeq 2300$ and 2500. They may coexist and puffs may temporarily grow while slugs may degenerate into puffs. The speed and growth rate measurements do not show any typical scaling at a critical point, such as the square-root scaling of the percolation type processes. The front speed of puffs just smoothly changes to that of slugs. All these facts make the transition scenario to fully turbulent flow unclear. In what follows, I will show that a perspective of viewing pipe flow as an excitable media, as proposed by Barkley (2011b), eventually helps to clarify this problem.

\subsection{Modelling puffs and slugs: Barkley's model}

In excitable media, excited states are bounded by sharp fronts. The dynamics of such fronts has long been studied with low dimensional model equations, mostly in chemical reactions and electrophysiological systems (see Tyson \& Keener (1988)). As discussed in Sec. 1.3, pipe flow bears a strong analogy to one-dimensional excitable media such as a nerve axon (see Fig. 1-7 and Fig. 1-8). The action potential of a nerve axon has long been modeled as reaction-diffusion systems (FitzHugh, 1961; Nagumo et al., 1962; Tyson \& Keener, 1988). Inspired by this analogy and the fact that on large scale pipe flow is rather one dimensional (fronts only move in one direction), Barkley (2011b) proposed to view pipe flow in the context of excitable and bistable media. As shown below that pipe flow indeed can be modeled by one-dimensional advectionreaction-diffusion equations, and its large scale behaviours such as puffs, transition 
from puffs to slugs and the growth of slugs can be very well captured. The chaotic and transient nature of puffs, puff splitting, and slugs with holes can also be successfully captured by either a discrete coupled map lattices type model (Barkley, 2011b) or ODE models with an additional noise term (Barkley, 2011a). However, a factor that will be demonstrated intrinsic to pipe flow, the nonlinear advection of turbulence, is missing in these models. It turns out that this nonlinear advection deprives these models of correctly predicting the scaling of the front speed as the flow transitions from puffs to slugs (private communication with Barkley). With the accurate (both DNS and experimental) measurements of the front speed of puffs/slugs, a new model proposed by Barkley that takes account for the nonlinear advection, correctly captures the scaling of the front speed in all flow regimes and predicts two types of slugs with different downstream fronts. In this section, this model will be introduced and the bifurcation scenario that gives rise to slugs and two types of slugs within the model will be discussed in details. A comparison between structures in the asymptotic limit and that from DNS will be made at the end of this section.

\subsubsection{The model equations}

In pipe flow, perhaps the most important two elements are turbulent fluctuations and the velocity (shear) profile, which interact nonlinearly with each other via the Navier-Stokes equations. Modeling aims to replace the full Navier-Stokes equations by simpler model equations with low-order nonlinearities whilst capturing the basic dynamics of the flow. Realizing that the velocity profile can be modeled by a scalar, the centerline velocity, Barkley (2011b) developed the two-variable one-dimensional model which successfully applied to pipe flow. By introducing a nonlinear advection, a new model is developed as the following:

$$
\frac{\partial q}{\partial t}+(u-\zeta) \frac{\partial q}{\partial x}=f(q, u ; r)+\Gamma \frac{\partial^{2} q}{\partial x^{2}}, \quad \frac{\partial u}{\partial t}+u \frac{\partial u}{\partial x}=\epsilon g(q, u)
$$

where variables $q$ and $u$ depend only on the streamwise coordinate $x$ and time $t$. $q$ models the level of turbulent fluctuations and $u$ models the centerline velocity of the flow. $u$ plays two important roles, it accounts for the nonlinear advection in the streamwise direction and it measures the state of the velocity (or shear) profile $(u=1$ for parabolic flow while $u<1$ for plug flow). Using the concepts from excitable media, $u$ is the control variable which control the level of $q$ and accounts for the refractory nature. $q$ is the trigger variable, or the propagator (Tyson \& Keener, 1988), that 
propagates through the system. The parameter $\zeta$ is a positive number and accounts for the fact that turbulence is advected more slowly than the centerline velocity, which was evidenced by our numerical simulation (see Appendix B). So, $u-\zeta$ is the nonlinear advection of the propagator $q$, as shown in the first equation of Eqs. (3.1). The parameter $\epsilon$ gives the ratio of time scales between the fast excitation of $q$ and the slow recovery of $u$ in the absence of $q . \Gamma$ is the diffusion coefficient of $q$ and is a constant. The functions $f(q, u ; r)$ and $g(q, u)$ account for the nonlinear interactions between $q$ and $u$ and should be modeled to capture the most fundamental features of pipe flow with minimal nonlinearities, where $r$ is a system parameter that corresponds to the Reynolds number in pipe flow and accounts for the transition from excitability to bistability.

Barkley proposed the following forms for $f(q, u ; r)$ and $g(q, u)$ :

$$
\begin{aligned}
f(q, u ; r) & =q\left(u+r-1-(r+\delta)(q-1)^{2}\right) \\
g(q, u) & =(1-u)-\rho q u
\end{aligned}
$$

where $\rho$ sets the ratio between the time scale at which $u$ decreases in response to $q$ and that at which $u$ recovers in the absence of $q . \delta$ is a small positive number that assures the linear stability of resting state for any value of $r$. If we consider only the local dynamics of the system Eqs. (3.1) (without spatial variations):

$$
\begin{aligned}
& \dot{q}=f(q, u ; r) \\
& \dot{u}=\epsilon g(q, u),
\end{aligned}
$$

then the local dynamics will be organized by the nullclines $f=0$ and $g=0$. Clearly the $q$-nullcline $f=0$ has three branches because $f$ is cubic in $q$, a trivial branch $q=0$ (denoted as $q^{0}$ ) and two non-trivial branches from a parabola, a stable upper branch $q^{+}$and an unstable lower branch $q^{-}$. This captures the basic features of subcritical shear flow that small amplitude perturbations will be damped out and flow falls back to laminar state, however, perturbations above a certain threshold will trigger turbulence. This unstable lower branch depicts such a threshold in $q$. The $u$-nullcline $g=0$ tells how $u$ decreases as the fluctuation level $q$ increases and the other way around. The parameter $r$ plays a role as the Reynolds number. At all $r$, the system has a stable fixed point $u=1 ; q=0$ corresponding to the resting state (laminar flow) and it will be the only fixed point when $r$ is small. As $r$ increases above certain point, $q$-nullcline moves such that the $u$-nullcline intersect the upper 
branch of $q$-nullcline $q^{+}$. By then a new fixed point other than $u=1 ; q=0$ appears in the $q-u$ phase space, which corresponds to a stable excited state, i.e., the stabilized turbulent flow. As this happens, the system becomes bistable and specifically in pipe flow transition from localized turbulence to expanding turbulence (the predecessor of fully turbulent flow) occurs. See Fig. 3-9)(b) for an example.

Fronts are the regions that connect the solutions on the branch $q^{0}$ and $q^{+}$, in
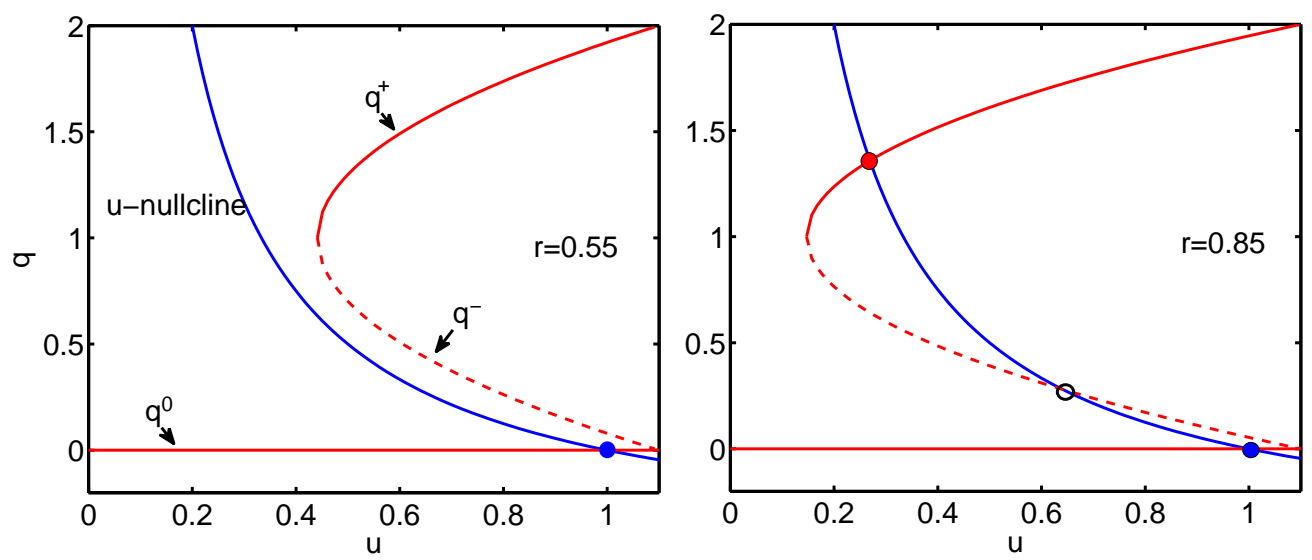

Figure 3-9: $u$-nullcline and $q$-nullcline in $q-u$ space. The $q$-nullcline (the two nontrivial branches) moves with the change in the parameter $r$. At low $r$ there is only one fixed point at $u=1 ; q=0$ (the blue dot) but a stable fixed point appears on the upper branch of $q$-nullcline as $r$ increases (the red dot).

pipe flow, they are the laminar-turbulent interfaces bounding the turbulent area. The standard asymptotic analysis of the fronts and the calculation of the front speed can be found in Tyson \& Keener (1988).

\subsubsection{Speed asymptotics}

Here I just summarize the core results of the asymptotic analysis of the front speed, for details see Tyson \& Keener (1988); Barkley et al. (2014). The asymptotic limit corresponds to $\epsilon \rightarrow 0$, i.e., sharp front case. In the situation of a front propagating in the system, the flow region can be divided into two regions, the outer region that corresponds to either the laminar solution $q^{0}$ or turbulent solution $q^{+}(u)$, and the inner region (within the front) with a width on the order of magnitude of $\epsilon$ that connects the laminar and turbulent solutions. In the limit $\epsilon \rightarrow 0, u$ does not change across the front because of the separation of time scales: $q$ changes fast across a front, while $u$ responses to $q$ much slower so that is constant to the leading order in $\epsilon$. The 
front speed in the asymptotic limit is given by

$$
c=u_{f}-\zeta \pm \sqrt{\Gamma} s\left(u_{f} ; r\right)
$$

where $u_{f}$ is the $u$ at the front, + corresponds to the downstream front and - the upstream front. $s$ is defined as $\frac{c-\left(u_{f}-\zeta\right)}{\sqrt{\Gamma}}$ and given by the non-linear eigenvalue problem

$$
q^{\prime \prime}+s q^{\prime}+f\left(q, u_{f}\right)=0
$$

subject to the boundary equations

$$
q(-\infty)=q^{0}, q(+\infty)=q^{+}\left(u_{f}\right)
$$

The shape of the front q can also be obtained from this equation.

The fact in pipe flow is that the upstream front of puffs/slugs is always a sharp front (see e.g. Wygnanski \& Champagne (1973); Wygnanski et al. (1975)) and the jump from $q^{0}$ to $q^{+}$is relatively abrupt at $u=1$, because $u=1$ upstream of the front and has not had changed across the sharp front. However, the drop (sharp in the asymptotic limit) from $q^{+}$to $q^{0}$ at the downstream front is not abrupt at low $R e$ and does not occur at $u_{f}=1$, it however becomes sharp in the large Re limit. Two kinds of slugs with different downstream fronts have also been documented by Duguet et al. (2010b). At $R e=3000$ there is not an intensity peak at the downstream front, while there is at $R e=4500$ (figure 13 and 16 in there). The one without an intensity peak will be referred to as a weak front, and the one with an intensity peak a strong front hereafter. Considering this fact, two choices of $u_{f}$ were proposed: for the weak front $u_{f}=u_{s s}$ where $u_{s s}$ is the $u$ at the upper stable fixed point (in the puff regime this fixed point does not exist), and for the strong front $u_{f}=1$ as the case for the upstream front. One may notice that the speeds of the upstream and strong downstream front are symmetric about the value $1-\zeta$ according to Eqs. (3.6), which will be called the neutral speed and denoted as $C_{N E}$ hereafter.

\subsubsection{Three types of structure in the asymptotic limit}

The above discussion leads to three kinds of structure, puff, slug with a weak downstream front (will be referred to as slug I), and slug with a strong downstream front (slug II). Here the shape of these three structures will be described.

1. Puffs, correspond to (A) and (D) in Fig. 3-10. Although there is no upper 

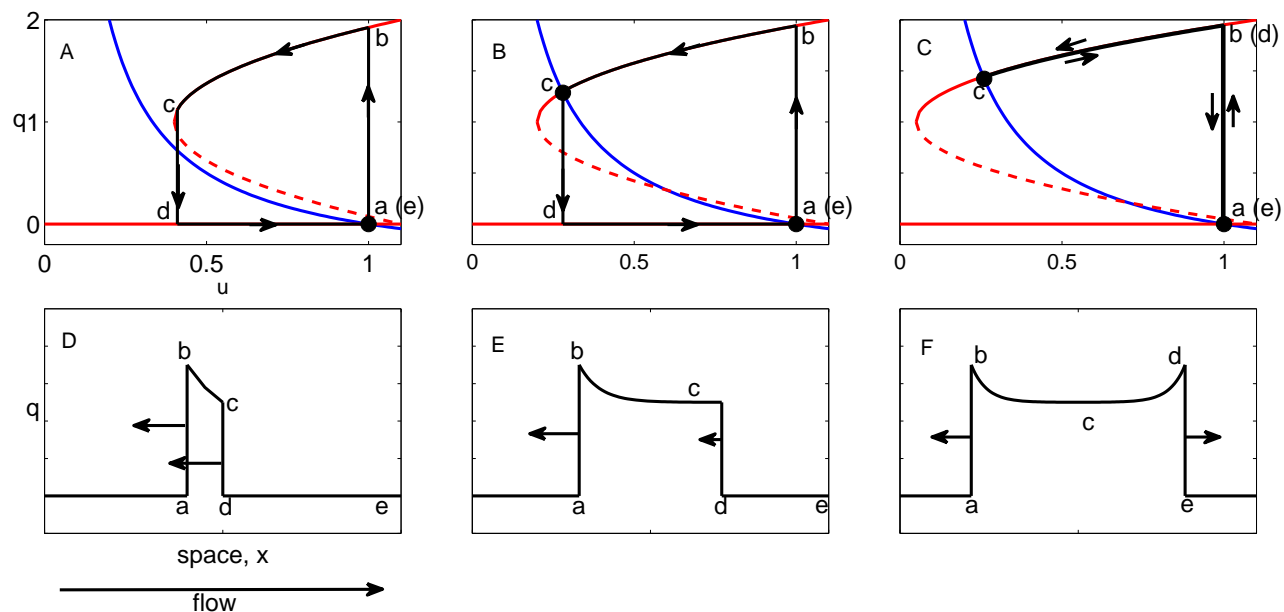

Figure 3-10: Model fronts of puffs, slug I and slug II in the asymptotic limit. (A)-(C) show fronts that connect the laminar branch $q^{0}$ and turbulent upper branch $q^{+}$in $q-u$ space. (D)-(F) show the shape of fronts and turbulence structures in $q$ - $x$ physical space, where the length of arrows represents the magnitude of the speeds. In physical space, if one goes from upstream to downstream along $a-e$ as shown in panel (D), the route in (A)-(C) follows the corresponding sequence (counterclockwise). All fronts and speeds are shown in a moving frame of reference with the neutral speed $C_{N E}$. In this frame, the upstream and downstream fronts speed are mirror reflection of each other for strong fronts, see panel $(\mathrm{F})$.

fixed point, system can still be locally excited to the upper branch $q^{+}$by finiteamplitude perturbations, forming an upstream front (the vertical arrow-lines $a-b$ in Fig. 3-10(A)) while $u$ stays unchanged across the front. Then the centerline velocity $u$ decreases in response to $q$ and the dynamics evolves on the upper branch $q^{+}(b-c)$ until it falls off and $q$ drops to 0 , forming the upstream front $(c-d)$. Finally, $u$ relaxes towards 1 and the velocity profile recovers to a parabola $(d-e) . q$ falls off the upper branch at a point such that the speed of two fronts are identical, giving rise to a fixed length of puffs. The shape of the puff in the asymptotic limit is shown in Fig. 3-10(D).

2. Slug I, corresponds to (B) and (E) in Fig. 3-10. The upstream front (vertical $a-b)$ is similar to that of puffs, however, a fixed point appears on the upper branch $q^{+}$where turbulence can stabilize, resulting in a plateau in the $q-x$ space as shown in Fig. 3-10(E). At relatively low Re, $q$ drops from this fixed point to $q^{0}$ at a certain distance downstream of the upstream front, forming a downstream front which is similar to that of puffs $(c-d)$. But, the speed of this front is determined by Eqs. (3.6) with $u_{f}$ taking the value at this fixed point and in general $C_{U F} \neq C_{D F}$. While for puffs, the downstream front just adjusts 
the $u_{f}$ to match the speed of the upstream front.

3. Slug II, corresponds to (C) and (F) in Fig. 3-10, At high Reynolds numbers, at the downstream front $q$ first climbs up on the upper branch $q^{+}(c-d)$ and drops to $q^{0}$ at $u=1(d-e)$, forming a front that is exactly the same as the upstream front (see the shape in panel $(\mathrm{F})$ ).

\subsubsection{Three types of structure from DNS}

The three types of structure predicted by the model, i.e., puff, slug I and slug II are indeed observed in real pipe data from DNS. Some space-time plots of these structures and the shape of the fronts are shown in Fig. 3-11. (a)-(c) show the space-

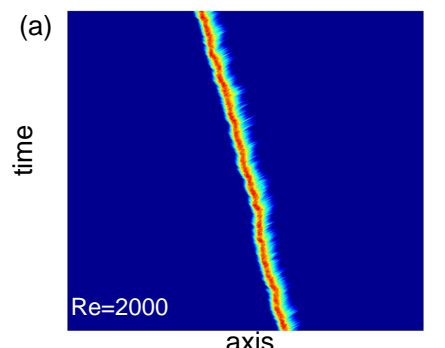

(d)
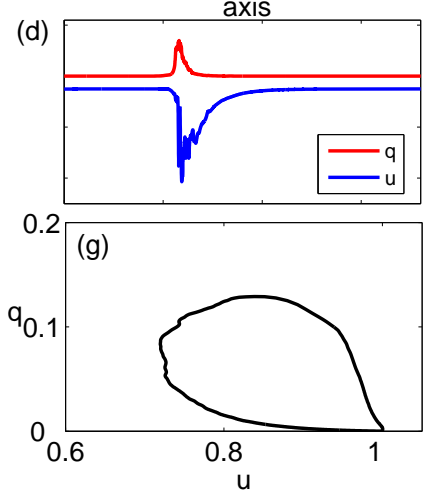

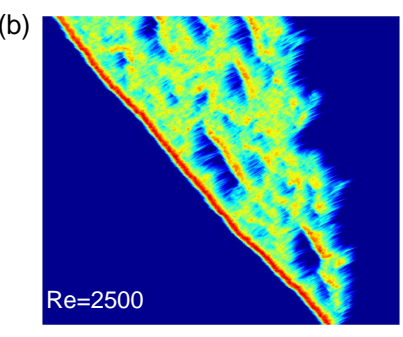

(e)

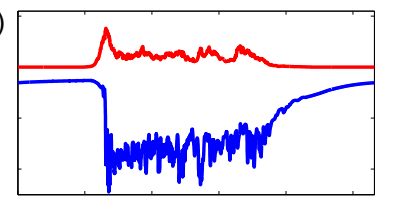

(h)

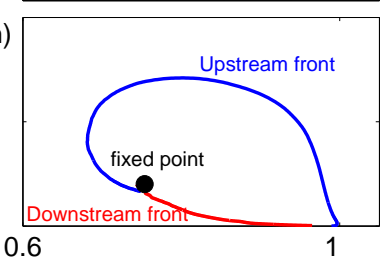

(c)

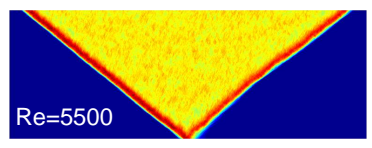

(f)

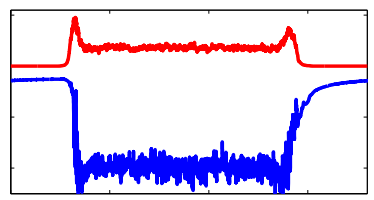

(i)

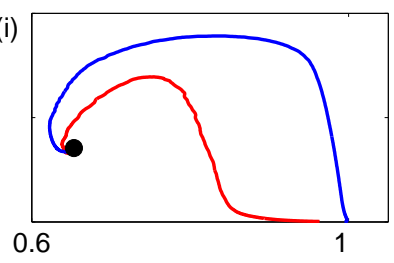

Figure 3-11: Three types of structure depicted by space-time plots of the local intensity $q(z)$ in a logarithmic color scale (a-c), distribution of the local turbulence intensity $q(z)$ (red lines) and the centerline velocity $u$ (blue lines) in (d-f), and the shape of the fronts (lines) and fixed point (black circle) in $q-u$ phase space in (g-i). Flow is from left to right and the space-time plots are in a frame of reference with the neutral speed $C_{N E}=0.53$ (see Barkley et al. (2014)). Left column is for a puff at $R e=2000$, middle column for a slug at $R e=2500$ and right column for a slug at $R e=5500$. The 'mean' front shape in (g-i) are averaged over time and all runs.

time plot of the three types of structure in the neutral frame of reference, i.e., the one moving at the neutral speed $C_{N E}=0.53$ (for details see Barkley et al. (2014)), at Reynolds number 2000, 2500 and 5500. The local intensity $q(z)$ is plotted along the pipe axis in a logarithmic color scale with blue representing laminar region and 
bright colors for turbulent area. (a) and (b) show a puff and a slug I with both upstream and downstream fronts moving upstream in this neutral frame. A slug II at $R e=5500$ (c) has a sharp downstream front depicted by the right-tilting red stripe. These observations agree well with the weak and strong fronts predicted by Barkley's model. (d)-(f) show the distribution of $q(z)$ along the pipe axis, which visualizes the shape of the structures in physical space and shows the difference in the fronts. As can be seen, the upstream front is always sharp and looks the same for puffs and slugs (see the red stripes in the space-time plots and the sharp peaks in $(\mathrm{d}-\mathrm{f}))$. However, the downstream front changes as Re increases, from a rather diffusive one for a puff, to a weak front that resembles puffs' front, and eventually to a strong front that is symmetric to the upstream front at high Reynolds numbers. Fig. 3-11(g-i) plot the fronts in $q-u$ phase space. To obtain these shapes, puffs were averaged over time and all runs at $R e=2000$ and are shown as a single black curve in (g). Slugs at $R e=2500$ and 5500 as shown in (h) and (i), were divided into three parts: the upstream front (blue), the turbulence core (the black circle), and the downstream front (red). These three parts were averaged respectively over time and all runs. The core of these slugs is a plateau on average, which corresponds to the stable fixed point on the upper branch $q^{+}$, and is only shown as a single black circle. These front shapes qualitatively agree with the three shapes predicted by the model in the asymptotic case, as shown in Fig. 3-10 (A-C). One difference is that the drop of $q$ at the downstream front is not sharp in real pipe flow at low Reynolds numbers (as will be shown in the next section, the speed of finite-width fronts can be well captured by the model with finite $\epsilon$ ). Instead, the downstream front of puffs and of slug I smoothly connects the turbulent state and the laminar flow. Nevertheless, the speed selection mechanisms are totally different according to the asymptotic analysis, as discussed in Sec. 3.2 .2 and Sec. 3.2.3

\subsection{Transition scenario in pipe flow}

Barkley's model is a generic model for a wide class of advection-reaction-diffusion systems and has a few parameters that can not be fixed without real data from specific systems. The DNS and experimental measurements provide accurate front speed data, which agree with each other very well and allow the model to select correct parameters so to capture the main features of pipe flow turbulence. The speeds from DNS, experiments, and the model are shown in Fig. 3-12. 
By choosing correct parameters, the model can very well capture the scaling of

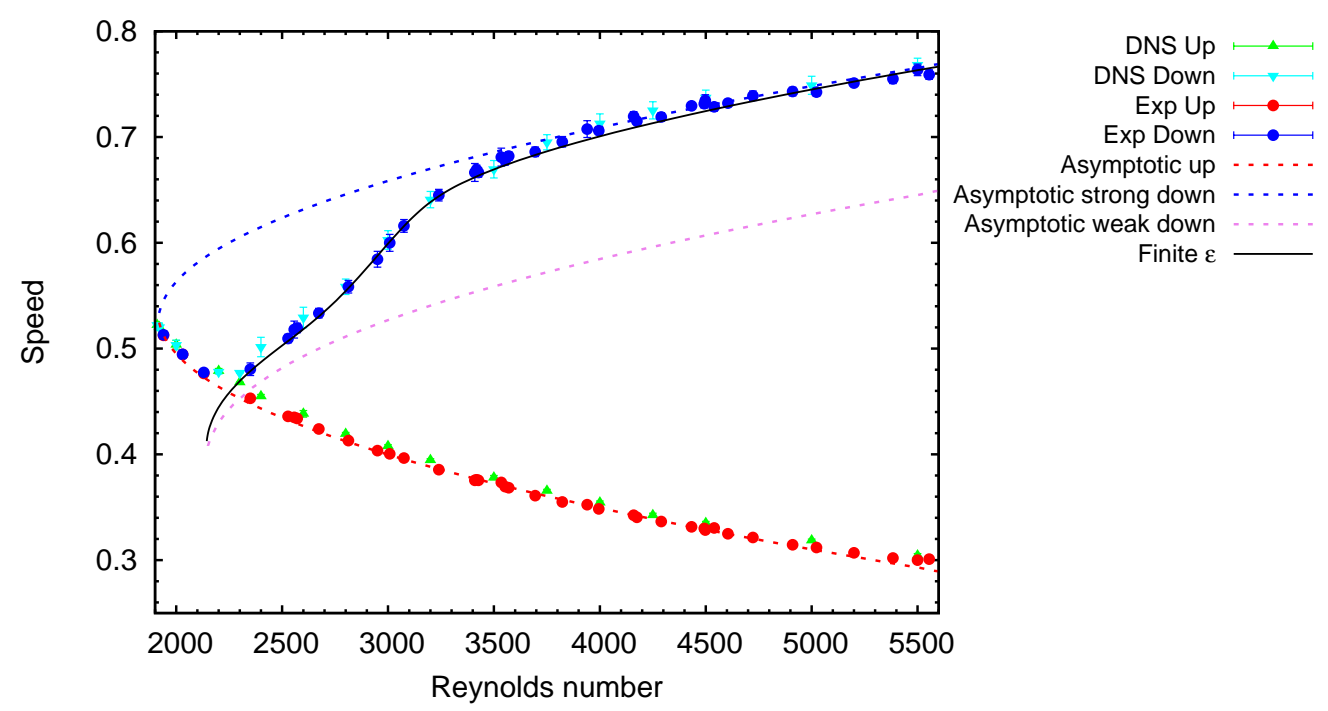

Figure 3-12: Comparison between between DNS, experiment and Theory (with the courtesy of Mukund and Barkley). Excellent agreement has been obtained.

the front speed of pipe flow turbulence, as shown by the good agreement between all three data sets in Fig. 3-12, The symbols are speeds from DNS and experimental measurements. The dotted lines are the speed in the asymptotic limit which form (branches of) parabolas. The black solid line represents the speed of the downstream front with finite $\epsilon(=0.2)$, i.e., the front with finite thickness, which is the case in real pipe flow (see Fig. 3-11).

The upstream front is always a strong front and the measured speed just falls on the asymptotics (the red dotted line in Fig. 3-12), however, the blue dotted line, which represents the speed of the strong downstream front, is not visited by the real pipe flow data at low and moderate Reynolds numbers $(<3500)$. This is a consequence of the fact that puffs do not have a strong downstream front and slugs at moderate Reynolds numbers also have rather diffusive downstream fronts (see Fig. 3-11). Instead, at low Reynolds numbers below about 2600, the downstream front speed is governed by the asymptotics for the weak front (the violet dotted line in Fig. 3-12), which infers that slugs at low Re fall in slug I. At high Reynolds numbers (above 3500) the measurements for both downstream and upstream fronts perfectly fall on the asymptotics for strong fronts, indicating slugs of type II. The data collectively demonstrate a smooth change of downstream front from a weak to a strong one as Re increases, which is captured by the finite- $\epsilon$ curve (the solid black line) from the model. The asymptotics for the upstream and strong downstream front are the reflection of each other about 
the neutral speed which was found to be $C_{N E}=0.53$. The downstream front speed of real pipe flow is regulated by the strong and weak asymptotics, with the speed smoothly swinging up from the weak branch to the strong branch as Re increases. No sharp transition was found. Because of this smooth change from the weak branch to the strong branch, two inflectional points appear on the downstream front speed curve following the twice curvature change (see the black line). This has not been observed in former sparser data sets (Lindgren, 1969; Wygnanski \& Champagne, 1973; Durst \& Ünsal, 2006; Nishi et al., 2008). No bifurcation point and critical scaling at a bifurcation point were observed. We argue that two factors may contribute to it.

The bifurcation point in the model, at which the system becomes bistable, is masked by the nonlinear turbulence advection. This can be best illustrated by the comparison between the speed asymptotics with and without non-linear advection, as the front speed of real pipe flow is regulated by these asymptotics. Fig. 3-13 shows the speed asymptotics in these two situations. In the presence of nonlinear advection,
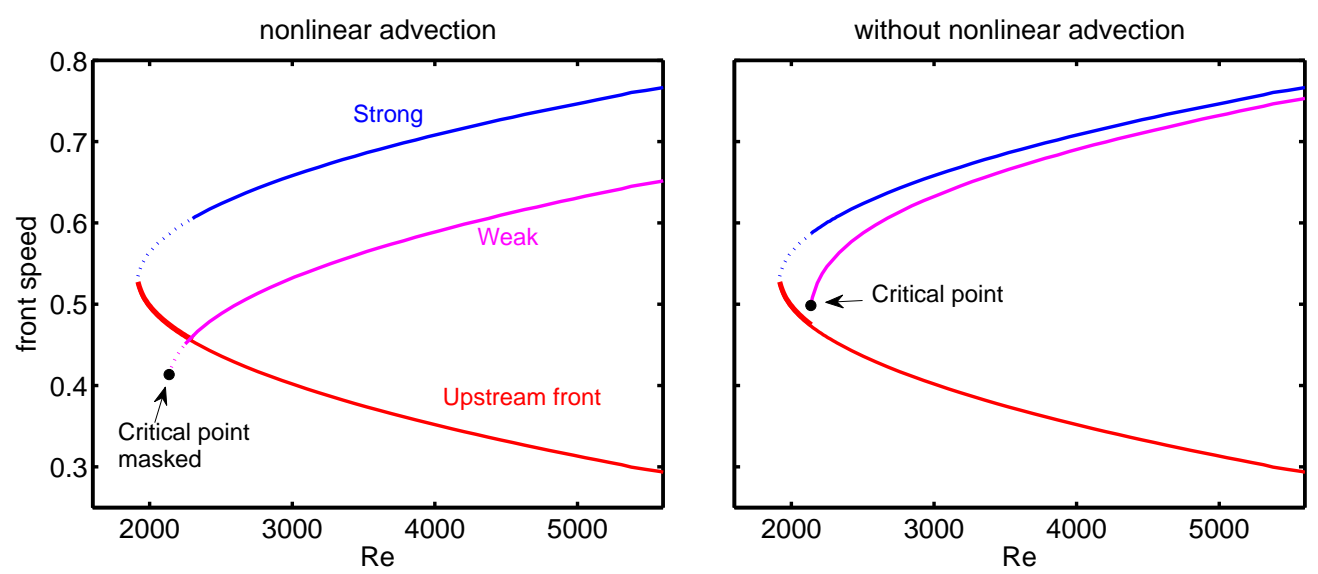

Figure 3-13: The speed asymptotics with and without nonlinear advection. Blue and violet lines for strong and weak downstream fronts and red lines for upstream front. The bifurcation point where the fixed point first appears on the upper branch $q^{+}$is denoted by a black circle.

the asymptotics for the weak downstream front crosses that for the upstream front at $R e \sim 2300$, see the intersection of the violet line and the red line in Fig. 3-13)(left). The part of the weak front together with the critical (bifurcation) point below the upstream branch is totally masked, as the structure with a downstream front slower than the upstream front is unstable and can not exist. Instead, $q$ drops from the upper branch $q^{+}$before it reaches the fixed point for the downstream front to match the speed of the upstream front. Turbulence appears as puffs whose downstream front simply follows the upstream front at a fixed distance. On the contrary, without 
nonlinear advection, the critical point is above the downstream front branch so that would not be masked. In this case, there would be a sharp transition in the front speed at the bifurcation point, which is in fact not observed in real pipe flow. In conclusion, the results indicate that this nonlinear advection, which has not been documented in other excitable media, masks the bifurcation point and is one of the reasons for the absence of typical behaviours near a bifurcation point.

Another factor is fluctuations. They are intrinsic to turbulence but are not considered in the current PDE model. In fact, fluctuations become significant at low Reynolds numbers between about 2300 and 3000. Fluctuations lead to puff splitting and dig holes inside slugs, and tend to obscure puffs and slugs in the regime $2300 \lesssim R e \lesssim 2600$. As a consequence, there is no clear distinction between puffs and slugs so that a critical point that clearly divides the two is absent.

Based on the turbulence and laminar length statistics, Avila \& Hof (2013) studied the intermittent nature of turbulence and proposed that there is only a universal turbulent state, which manifests strong fluctuations (spatiotemporal intermittency) at low Reynolds numbers and rare intermittency at high Reynolds numbers. For example, the space-time plot in Fig 3-11(b) shows a slug with holes (low turbulent intensity wells or even laminar gaps), which can be attributed to fluctuations. This strong spatiotemporal intermittency can be observed up to $R e \simeq 2800$ (Avila \& Hof, 2013). In fact, fluctuations can be incorporated in models. Barkley (2011b) captured this intermittent nature of pipe flow with a discrete model, where the $q^{+}$nullclines was replaced by a wedge-shaped chaotic area that local dynamics can escape from. Alternatively, Barkley (2011a) showed that by adding a noise term to the PDE model (Barkley, 2011b), the equations could also capture the puff splitting and holes inside slugs. However, these fluctuations make the theoretical understanding of the transition scenario difficult.

The DNS simulations here show that at some Reynolds numbers fluctuations may even cause transient changes in the properties of the downstream front of slugs. Here a space-time plot at $R e=3000$ illustrates such a case in Fig. 3-14. The spacetime diagram (a) shows the switching between weak and strong downstream fronts, where the weak front is almost vertical and strong fronts manifests itself as a right tilting red stripe. The tilting to the right indicates that the speed of the strong front is higher than the neutral speed $C_{N E}$. On the right, in Fig. 3-14(b) the speeds of these two fronts are plotted on the speed diagram and they almost perfectly lie on the speed asymptotics from the theory. The front transiently takes one of the two forms and result in an overall mean sitting between the speeds of these two kinds of front (the 
(a)

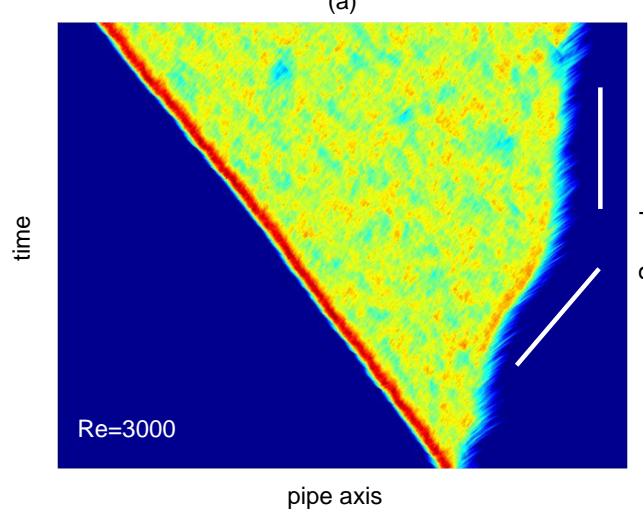

(b)

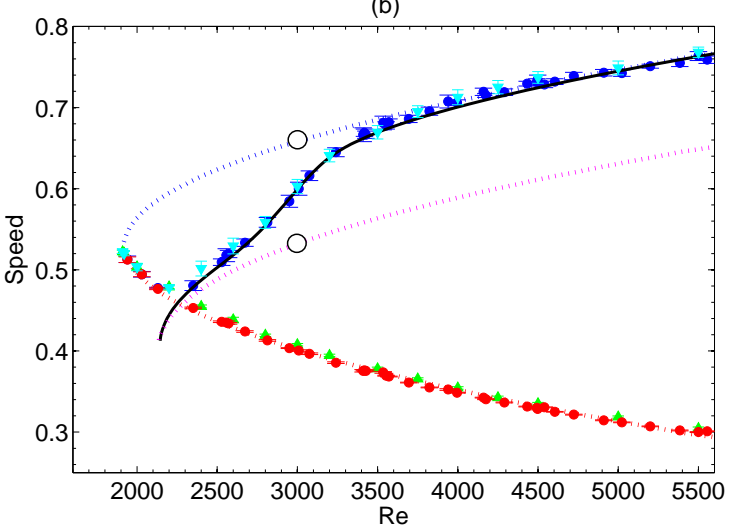

Figure 3-14: The transient switch between weak front and strong front at $R e=3000$. (a) The space time plot. The two white bars shows the slope of the weak and strong fronts, i.e., the speed. (b) the speed of the weak and strong front shown as two white circles, corresponding to the slope of the white bars in (a).

cyan symbol between the two white circles in Fig 3-14(b)). While the model does not capture the instantaneous dynamics which are strongly influenced by statistical fluctuations, it does capture the average quantities (e.g., see the black curve in Fig. 3-12) and explains the underlying bifurcation scenario leading to fully turbulent flow.

The transition scenario was drawn as the following. At low Reynolds numbers (below $\sim 2200$ ) pipe flow is excitable and turbulence takes the form of localized turbulence, i.e., puffs. As Re increases the system becomes bistable, turbulence can locally stabilize and slugs start to emerge. Turbulence first takes the form of slugs with weak downstream front, and smoothly picks up a strong downstream front as $R e$ increases above $R e \simeq 3500$. No bifurcation point was found because the nonlinear advection and intrinsic fluctuations mask the bifurcation point. Fluctuations also contribute to the smooth change of weak downstream fronts to strong ones for slugs.

\subsection{Discussion}

In this chapter, one of the central problems in fluid dynamics that how fully turbulent flow arises, was investigated in great details. In order to study the transition from puffs to slugs, the speed of the laminar/turbulent fronts was measured in long pipes at a variety of Reynolds numbers $R e \in(19105500)$ with DNS. The speed agrees well with accurate experimental measurements from my colleagues. The unprecedentedly accurate data from both DNS and experiments allow a generic advection-reaction-diffusion 
model developed by Barkley (Barkley et al., 2014) to fit the model parameters and capture the general features of pipe flow in all flow regimes. The asymptotic analysis of the front predicted three types of structures on the route to fully turbulent flow: puffs, slugs with a weak downstream front, and slugs with a strong downstream front, which were all observed in DNS and shown to have the properties predicted by the model. The smooth change from a weak downstream front to a strong one, as a consequence of fluctuations, was very well captured by the finite $\epsilon$ solution from the model. Collectively, these results showed that pipe flow undergoes a smooth transition from excitability to bistability, where puffs are localized excitations that feed on the surrounding laminar flow and eventually turbulence arises as the second stable state. No bifurcation point and typical behaviours at a bifurcation point were observed. Neither the front speed nor the growth rate bears a square-root scaling as in the percolation type of non-equilibrium phase transition. The nonlinear turbulence advection and intrinsic fluctuations mask the bifurcation point, depriving the system of a critical scaling at the onset of fully turbulent flow, which caused great difficulties in earlier relevant studies (e.g. Lindgren (1969); Wygnanski \& Champagne (1973); Durst \& Ünsal (2006); Nishi et al. (2008)).

Besides, the transition scenario also suggests possibilities of turbulence control that forces the inverse transition. In the model system, the fully turbulent state corresponds to a fixed point on the upper branch $q^{+}$, which allows spatially extended turbulence to stabilize. If, by some control mechanisms, the nullclines can be changed such that the fixed point disappears, the bistable system will revert to excitable system. Since in an excitable system turbulence can exist only in localized form, great friction drag can be achieved if fully turbulent flow degenerates to localized puffs. Further, the excitable media requires the control variable to sufficiently recover for supporting new excitation, this suggests that the system can be rendered refractory if the control variable is controlled below the value that excitation requires to survive. In next chapter, this possibility will be explored and implemented. 


\section{Chapter 4}

\section{TURBulenCE CONTROL}

The previous chapter deals with the transition to fully turbulent pipe flow. In this chapter the opposite problem will be addressed: how to relaminarise fully turbulent flow, or in other words, how to force an inverse transition at high Reynolds numbers. This study is motivated by the fact that in practice flows at high Reynolds numbers are usually turbulent despite the linear stability of the laminar flow. Turbulence at high Reynolds numbers causes much higher friction loss than laminar flow due to its highly dissipative nature, and hence the energy consumption for fluid transport and vehicle propulsion is significantly increased.

In the previous chapter, it was clarified that the transition to fully turbulent pipe flow is a transition from excitability to bistability. As Re increases, a fixed point appears in the system aside from the laminar flow, which results in the stabilization of spatially extended turbulence. Naturally, one may infer that if this fixed point can be removed from the system with some control strategies, turbulence will undergo inverse transition to a localized state which will eventually decay due to its transient nature.

First recall the nullclines of the model system explained in Sec. 3.2.1. The $q^{+}$ branch crosses the $u$-nullclines above the bifurcation point. If the nullclines can be shifted by some control strategies, this fixed point may be removed and the system may become excitable or even refractory. Considering that in this model system, there are only two variables $q$ and $u$, which represent turbulent fluctuations and the centerline velocity, or more fundamentally the shear profile, clearly the centerline velocity is an easier target than the fast fluctuations on small length scales from the point of view of control. To some extent, this strategy has been implemented by Hof et al. (2010) where the localized puffs were successfully eliminated by a modification of the velocity profile at the rear of puffs. A body force was imposed at the rear of puffs, 
decelerating the flow at the pipe center and accelerating the flow close to the wall. The velocity profile was modified into a plug-like one and puffs relaminarised. It will be shown below that in the model system, such a strategy corresponds to a change of the nullclines of the system, see Fig. 4-1.

In case of localized puffs, the forcing changes the $u$-nullcline but does not affect

(a)

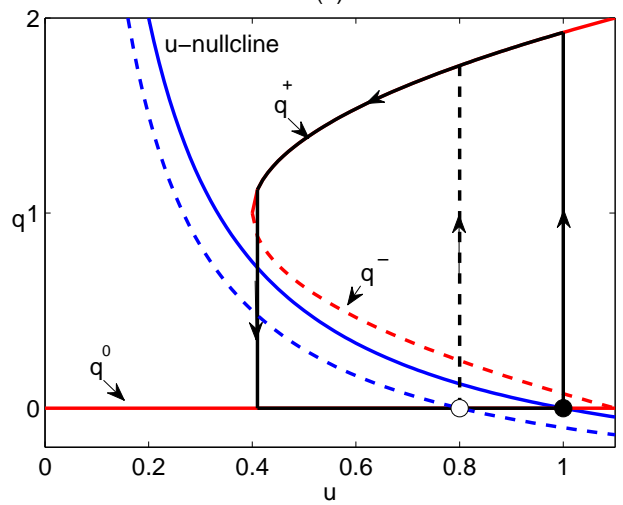

(b)

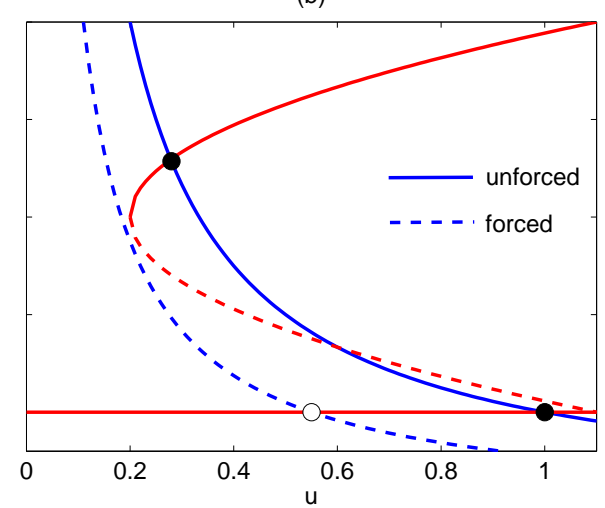

Figure 4-1: The change in the nullclines in case of forcing.

the $q$-nullcline as it only directly acts on $u$ but not on $q$. It decreases the centerline velocity such that when $u$ relaxes to the resting state in the absence of $q$, it no longer relaxes to 1 but to some lower value, for example 0.8 as shown in Fig. 4-1(a). In the forced case, the upstream front no longer occurs at $u_{f}=1$ but 0.8 because the velocity profile upstream of it can not fully recover, consequently the speed of the upstream front also changes. The downstream front will in principle also change the position (not shown in the figure) on the upper branch $q^{+}$so to match the speed of the upstream front. However, when the force is strong enough, i.e., the upstream front is shifted to the left sufficiently, the downstream front speed may not be able to match the upstream front speed anymore and causes puffs to collapse. Similarly, for the bistable case at higher Reynolds number, as shown in Fig. 4-1(b), a forcing may push the $u$ nullcline to the left and remove the fixed point on the upper branch $q^{+}$and cause an inverse transition from bistability to excitability. Spatially extended turbulence is no longer a stable state, hence, will collapse and degenerate to localized states, or simply completely relaminarise given sufficiently strong force.

Following this line of argument it may hence be possible to intercept the turbulence self-sustaining mechanism (Hamilton et al., 1995; Waleffe, 1997; Jimenez \& Pinelli, 1999) and to relaminarise turbulent flow using a suitable deformation of the velocity profile. The main topic of this chapter is to test forcing strategies in fully turbulent 
flow at high Reynolds numbers and gain a better understanding to the near-wall turbulence self-sustaining process. The Reynolds numbers, domain size, and resolution considered in this study are shown in Tab. 4.1.

\begin{tabular}{llllll}
\hline$R e$ & $R e_{\tau}=\frac{u_{\tau} R}{\nu}$ & $L_{z}$ & $N$ & $M$ & $K$ \\
\hline 3000 & 109.1 & $32 \pi$ & 64 & 48 & 640 \\
4000 & 141.1 & $8 \pi$ & 80 & 64 & 192 \\
4500 & 157.2 & $8 \pi$ & 80 & 64 & 192 \\
5000 & 172.8 & $8 \pi$ & 80 & 64 & 192 \\
7500 & 245.1 & $4 \pi$ & 128 & 128 & 128 \\
10000 & 315.1 & $4 \pi$ & 144 & 144 & 144 \\
\hline
\end{tabular}

Table 4.1: The domain size and resolution for the simulation at all the Reynolds numbers considered, in which $R e_{\tau}$ is the friction Reynolds number, $u_{\tau}$ the friction velocity, and $N$ the number of grid point in the wall normal direction. Note that in physical space there are $3 K$ and $3 M$ grid points in axial and azimuthal directions, and the domain size in wall unit is $L^{+}=L_{z} R e_{\tau}$.

\subsection{Forcing experiments}

In the following I introduce a volume force that flattens the mean velocity profile, then test the effect of the force on fully turbulent flow as a function of Reynolds number. Starting with a fully turbulent velocity field, this force is activated gradually until it reaches the desired amplitude. The simulation stops if the flow relaminarises, or the time exceeds a predefined maximum time $t_{\max }=4000$. At each $R e$ different force amplitudes will be tested in an attempt to find the critical force amplitude, with which the flattened velocity profile cannot sustain turbulence anymore and finally the flow reverts to the laminar state.

\subsubsection{Forcing}

An incompressible fluid in a pipe is subject to a driving force (usually the pressure gradient required to maintain fixed flux in pipe flow) and no-slip boundary conditions. The mean velocity profile can be manipulated by adding a body force in addition to the pressure. A radially dependent body force $\boldsymbol{F}=F(r) \hat{\boldsymbol{z}}$ is considered. $\boldsymbol{F}$ will be chosen such that the streamwise velocity decreases in the pipe center and increases near the pipe wall, while the mass flux is kept fixed. Now the Navier-Stokes equations become

$$
\frac{\partial \boldsymbol{u}}{\partial t}+\boldsymbol{u} \cdot \boldsymbol{\nabla} \boldsymbol{u}=-\nabla p+\frac{1}{R e} \Delta \boldsymbol{u}+\boldsymbol{F}, \quad \boldsymbol{\nabla} \cdot \boldsymbol{u}=0
$$


which will be used to determine the specific form of the force once the target velocity profile is given. At first an $\boldsymbol{F}$ is chosen such that the force deforms the velocity profile of laminar flow into a chosen target velocity profile, which is flatter and more pluglike than the parabola. The shape of this target velocity profile may be freely chosen. Once the target velocity profile $\boldsymbol{u}_{\beta}$ is completely determined, the desired force $\boldsymbol{F}$ is easily obtained by substituting $\boldsymbol{u}_{\beta}$ in Eqs. (4.1). In this thesis I choose a family of profiles parametrized with a parameter $\beta$ as following,

$$
\boldsymbol{u}_{\beta}(r)=(1-\beta)\left(1-\frac{\cosh (c r)-1}{\cosh (c)-1}\right) \hat{\boldsymbol{z}}
$$

The parameter $\beta$ is chosen to be the centerline velocity difference between the laminar profile and the target profile, which can be considered as a measure of the amplitude of the force (note that $\beta$ is normalized as velocity is, by $U_{\max }$, unless explicitly stated). Given $\beta$, the parameter $c$ is determined by imposing the aforementioned constant mass-flux condition

$$
\frac{1}{\pi} \int_{0}^{2 \pi} \int_{0}^{1} u_{\beta}(r) r d r d \theta=\frac{1}{2}
$$

As an example, the velocity profiles of the unforced laminar flow, and that of the forced laminar flow at $R e=3000$ with $\beta=0.2$ are compared in Fig. 4-2.
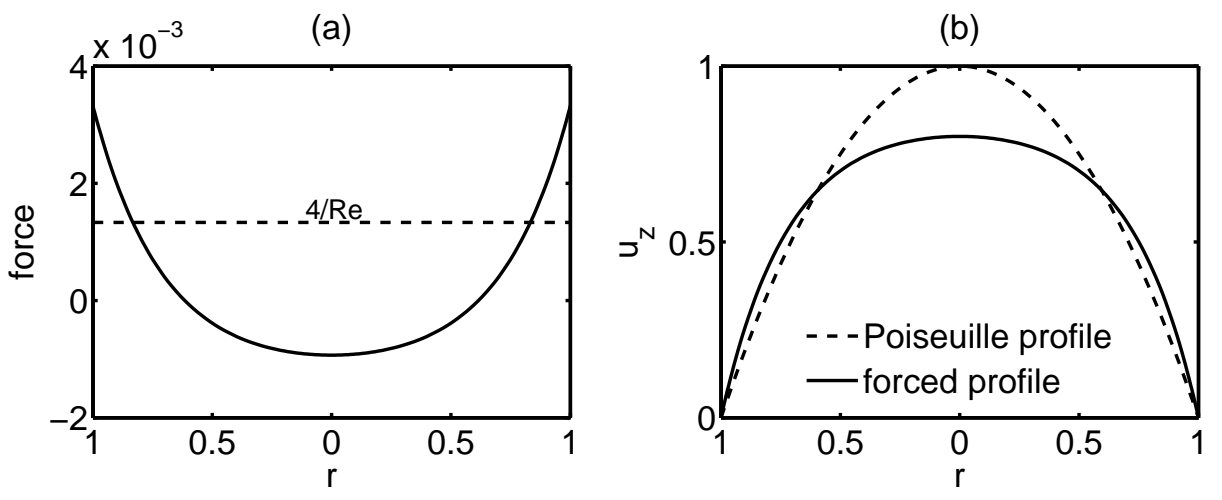

Figure 4-2: (a) The shape of the force chosen to decrease the centerline velocity of the laminar flow by $20 \%$ at $R e=3000$. The force is negative (pointing upstream) in the center and positive (pointing downstream) near the wall. For comparison, the pressure gradient in a laminar flow $\left|\frac{\partial p}{\partial z}\right|=4 / R e=1.33 \times 10^{-3}$ (the dashed line) is also shown. (b) Comparison of the parabolic profile and the forced profile of laminar flow at $R e=3000, \beta=0.2$. 


\subsubsection{Response of fully turbulent flow to the forcing}

Upon turning on the force, the turbulence undergoes a sharp decrease in the enstrophy associated with the streamwise vorticity, as shown in Fig. 4-3)(a). This indicates that this force indeed suppresses turbulence, and may or may not remove turbulence completely depending on the forcing amplitude and the specific details in initial conditions. A possible explanation of the turbulence suppression is that in the reduced mean shear streamwise vortices of a certain amplitude can only generate comparatively weaker streaks, which in turn through nonlinear self interaction generate weaker streamwise vortices according to the turbulence self-maintaining mechanism, reducing the turbulence intensity. To look deeper into this dynamics, the effect of the force on the vorticity is analyzed. The equations governing the vorticity $\boldsymbol{\omega}$ are

$$
\frac{\partial \boldsymbol{\omega}}{\partial t}+(\boldsymbol{u} \cdot \boldsymbol{\nabla}) \boldsymbol{\omega}-(\boldsymbol{\omega} \cdot \boldsymbol{\nabla}) \boldsymbol{u}=\frac{1}{R e} \Delta \boldsymbol{\omega}+\boldsymbol{\nabla} \times \boldsymbol{F},
$$

Since the force $\boldsymbol{F}$ is in axial direction and only radially dependent, i.e., $\boldsymbol{F}=F(r) \hat{\boldsymbol{z}}$, one can easily work out the last term in Eqs. (4.4)

$$
\boldsymbol{\nabla} \times \boldsymbol{F}=-\frac{\partial F(r)}{\partial r} \hat{\boldsymbol{\theta}}
$$

which only appears in the azimuthal component of the vorticity equations Eqs. (4.4). The force does not directly contribute as a source term to the generation of streamwise vorticity. Instead, it depresses the streamwise vorticity generation indirectly, via the convection and stretching of the vorticity, represented by terms $(\boldsymbol{u} \cdot \boldsymbol{\nabla}) \boldsymbol{\omega}$ and $(\boldsymbol{\omega} \cdot \boldsymbol{\nabla}) \boldsymbol{u}$ in Eqs. (4.4) respectively.

To evidence the suppression of the steamwise vorticity generation, forcing experiments are conducted on fully turbulent flow at $R e=4000$ with a forcing parameter $\beta=0.22$, and the effects of this force on vorticity dynamics is observed. Two time series of the value $\int_{V} \omega_{z}^{2} d V$ in the whole domain and axial distributions of the quantity $\iint \omega_{z}^{2} r d r d \theta$, which are considered as measures of the intensity of the streamwise vorticity, at several time instants are plotted in Fig. 4-3)(b). To examine the dynamics, the radial distribution of the $\omega_{z}^{2}$ and $\omega_{\theta}^{2}$ integrated over $z$ and $\theta$ are compared at several instants (see Fig. 4-4). From Fig. 4-4(a), one can see that the region of most vorticity is approximately $r \in(0.5,0.9)$ (in wall unit $y^{+} \in(14,70)$, the buffer layer and the lower logarithmic layer, consistent with the autonomous region found in channel flow by Jimenez \& Pinelli (1999)), where most of streamwise vortices are located. This region can be interpreted as the near wall region where vortices and 

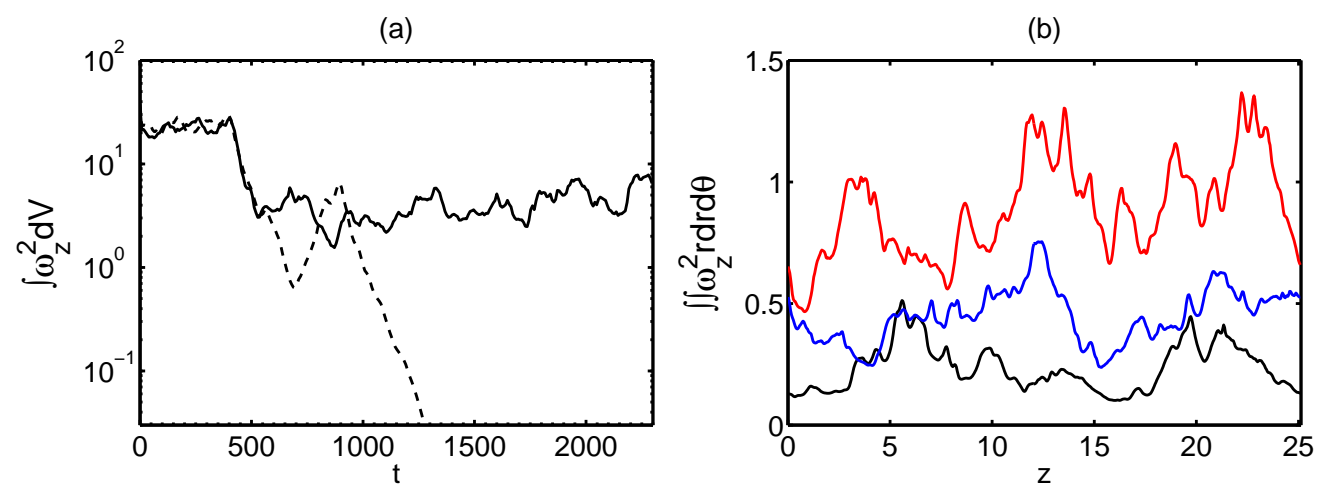

Figure 4-3: (a) Time series of $\omega_{z}^{2}$ integrated over the whole domain for two runs, the force is switched on at $t=400$. O ne survives under the force up to about 2400 and the other relaminarises more or less directly. Parameters for these two runs: $R e=4000$, pipe length $L_{z}=8 \pi$, and forcing parameter $\beta=0.22$. (b) Axial distribution of $\omega_{z}^{2}$ integrated over cross sections at three time instants: $t=200$ (red), 448 (blue), and 500 (black), from the solid line in (a).

(a)

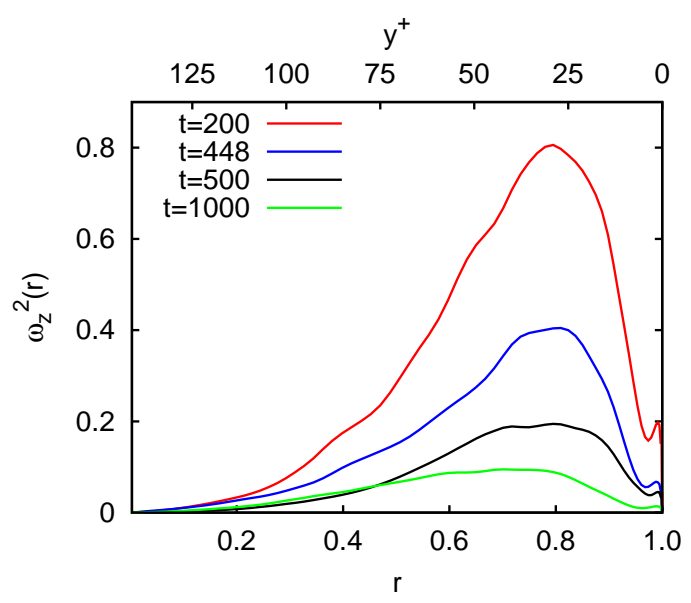

(b)

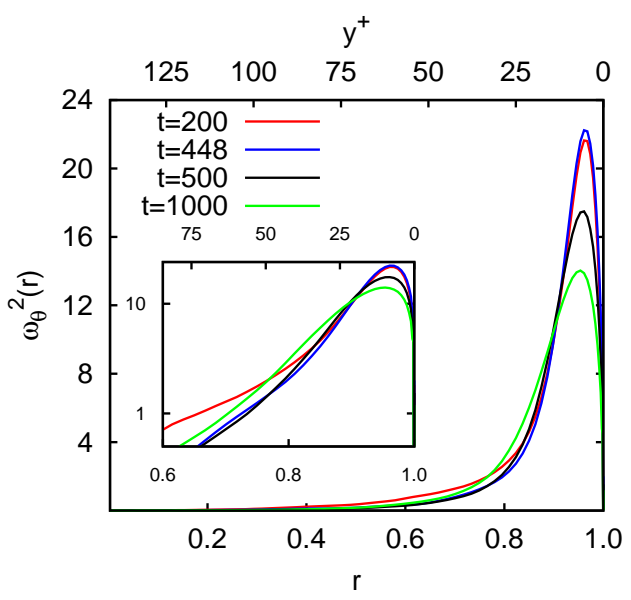

Figure 4-4: $\omega_{z}^{2}(\mathrm{a})$ and $\omega_{\theta}^{2}(\mathrm{~b})$ integrated over $z$ and $\theta$ as a function of radial position $r$. At each radial position, the ordinate represents the contribution of a cylindrical shell located at this radial position to the volume integral $\int_{V} \omega_{z}^{2} d V$. These four lines are taken at 4 time instants in the time series represented by the solid line in Fig. 4-3)(a), at $\mathrm{t}=200$ (blue), 448 (red), 500 (black), and 1000 (green). On the top axis the radial position $y^{+}$in wall units (measured from the wall) is also plotted. The inset in (b) is a zoom-in window with logscale in y-axis

streaks interact with each other, sustaining turbulence. Clearly, the generation of the streamwise vorticity is effectively suppressed in the presence of the force, both temporarily and spatially. The level of streamwise vorticity decreases considerably in the area $r>0.3$, as shown in Fig. 4-4(a). In Fig. 4-4(b), the $\omega_{\theta}^{2}$ as a function of $r$ can be interpreted as a measure of the mean shear. After the force is turned on, 

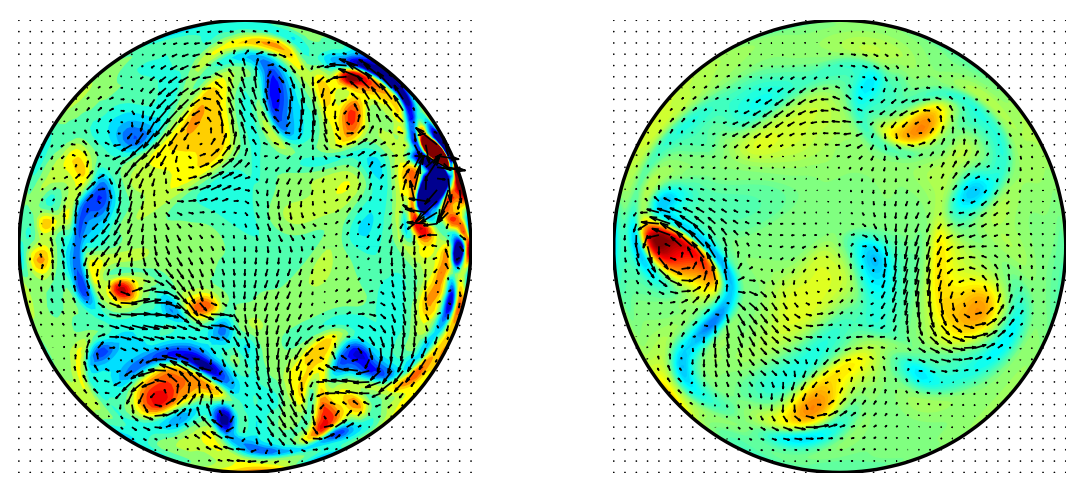

Figure 4-5: Contour of streamwise vorticity $\omega_{z}$ and in-plane velocity field plotted on a cross section at two time instants: $t=200$ (left) and 1000 (right) from the time series represented by the solid line in Fig. 4-3(a). They are taken at the most vortical area in the pipe at respective instant, where $\iint \omega_{z}^{2} r d r d \theta$ is at a maximum.

the mean shear slightly increases in the sublayer $\left(y^{+}<10\right)$ but decreases in the area $\left(y^{+} \in(14,70)\right)$, which can be seen in the comparison of the red line $(t=200)$ and the blue line $(t=448)$. Afterwards, the turbulence intensity decreases and the mean shear approaches that of the laminar profile, so that the mean shear ends up being lower in the sublayer but slightly higher above the sublayer compared to the unforced case (see the green and the red lines). The changes in the flow field are depicted in Fig. 4-5, where the suppression on the streamwise vortices due to the force is very clear.

For small forcing amplitude $\beta$, the flow remains turbulent, although turbulence does experience a decrease in its kinetic energy. When $\beta$ is increased, relaminarisation is observed. However, in certain range of $\beta$, turbulence may either completely decay or survive at a lower kinetic energy level after the initial decrease in its intensity depending on the specific initial conditions. This sensitive dependence of the final flow state on the initial condition is also clearly shown in Fig. 4-6, and implies that in marginal forcing amplitude regime, besides the shape of the velocity profile, the details of the flow field, such as the positions and sizes of the vortices, also affect the maintenance of turbulence. However, it is found that with sufficiently large parameter $\beta$, the forcing always eliminates turbulence directly. For example, at $R e=3000$ and under the force with amplitude $\beta=0.20$, the fully turbulent flow relaminarised in all 10 runs studied here.

Note that although the flow is forced here, the mean velocity profile of the turbulence is by no means fixed. A fixed mean velocity profile means imposing a time- 


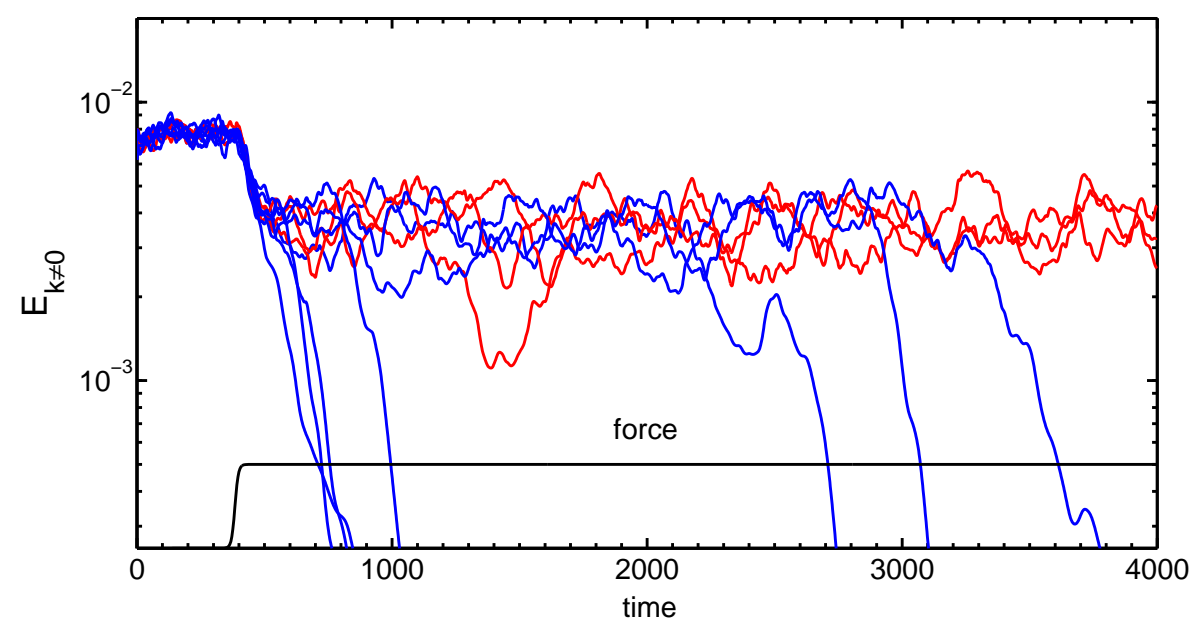

Figure 4-6: The time series of the kinetic energy of $z$-dependent turbulent fluctuations $E_{k \neq 0}$ for 10 runs with parameters $R e=4000$ and forcing amplitude $\beta=0.22$ in a $L_{z}=8 \pi$ pipe. The force (normalized by its desired amplitude) is turned on at $t=400$, as shown by the black line. The criterion for relaminarisation is $E_{k \neq 0}<2.5 \times 10^{-4}$, below which the turbulence is believed to be beyond recovery. Energy is normalized by the total kinetic energy of laminar flow in the same pipe. There are 7 relaminarisation cases (denoted by blue lines) out of 10 runs.

dependent but axially and azimuthally invariant force which sets the time derivative of the 0-0 mode of axial velocity to zero as explained in (Tuerke \& Jimenez, 2013). It was found that a fixed 'unnatural' mean profile breaks the equilibrium of radial momentum transport of turbulence, resulting in significant changes in turbulence intensity, but no relaminarisation was reported in their paper. In this work, the force is time-independent once it reaches the desired amplitude.

At the beginning of this chapter, we speculated that forcing may remove the fixed point that corresponds to the fully turbulent flow so that revert pipe flow from bistable to excitable. If this speculation is correct a full reverse transition including the reappearance of puffs, should be observed. For the example shown in Fig. 4-3(b), however, the domain size was too small to allow for localized puffs. An example in a $L_{z}=32 \pi$ pipe at $R e=3000$ with a $\beta=0.15$ force is investigated. Fig. 4-7 shows the space-time plot of the local turbulence intensity $q(z)$ (left) together with the comparison between the flow states at $t=0$ and 2000 (right). Clearly fully turbulent flow indeed becomes localized after the force is activated and eventually degenerates to a puff, which closely resembles that of puffs observed in normal flow at much lower $R e$ with a sharp upstream front and a more diffusive downstream front. 
This is a clear indication of changes in the 'nullclines' of the system, which is in
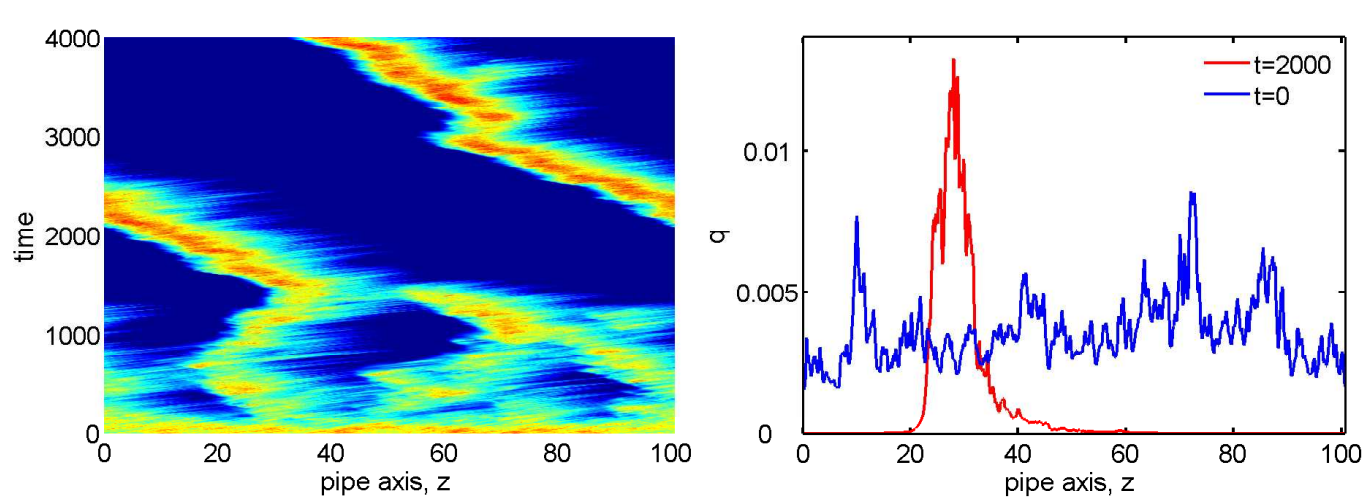

Figure 4-7: The localization of turbulence under forcing at $R e=3000$. The left is the spacetime plot of $q(z)=\iint\left(u_{r}^{2}+u_{\theta}^{2}\right) r d r d \theta$ in a frame of reference moving with the mean flow, at $t=0$ flow is fully turbulent and force is activated at $t=40$. On the right, the $q(z)$ is plotted along the pipe at $t=0$ and $t=2000$.

excellent agreement with the speculation at the beginning of this chapter. This result strongly supports the theoretical interpretation of the transition to fully turbulent pipe flow as a transition from excitability to bistability as discussed in Chapter 3 .

\subsubsection{Statistical investigation and critical forcing amplitude}

For the purpose of turbulence control, a minimum effort that suffices to remove turbulence is desirable. Here, this minimum effort is given by the weakest force that relaminarises turbulence. The minimum forcing parameter $\beta$ that suffices to eliminate turbulence, defined as $\beta_{c}$ hereafter, is to be determined. Considering the stochastic behaviour of the flow under forces with marginal amplitudes, for each point $(R e, \beta)$, 10 runs with different but statistically identical initial conditions are executed. These initial conditions are taken from a long time series of turbulence simulation and consecutive snapshots are separated by a time interval about 200, which is considered long enough for them to be statistically uncorrelated. Because of the stochastic dynamics of turbulence it is not possible to find a precise critical forcing amplitudes above which the flow always relaminarises and otherwise always stays turbulent. Therefore, here the critical amplitudes is defined as the one at which the probability of relaminarisation is equal to that of turbulence surviving, i.e. $\beta_{c}=:\left\{\beta \mid P_{\text {relaminarisation }} \simeq 0.5\right\}$, within 4000 time units. Considering the stochastic behaviour of turbulence under marginal forcing as shown in Fig. 4-6, one should perform lifetime statistics to accurately determine the critical forcing amplitude $\beta$ with which the lifetime of forced 


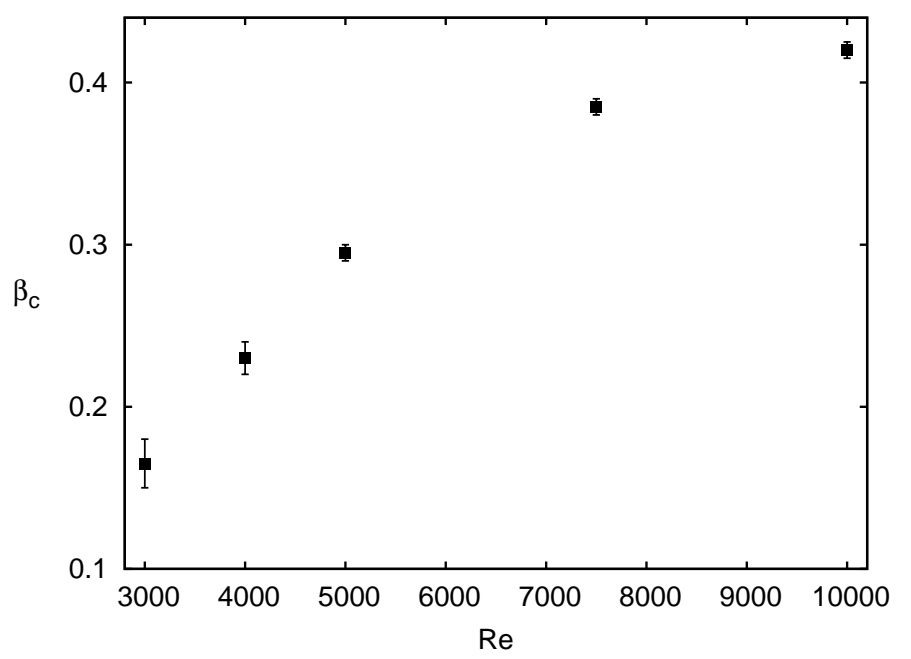

Figure 4-8: The dependence of the critical forcing parameter $\beta_{c}$ on Reynolds number $R e$. The upper bounds and lower bounds of each bar correspond to about $80 \%$ and $20 \%$ relaminarisation rate respectively.

turbulence for the first time diverges. However, given high Reynolds numbers and many $\beta$ 's to explore, lifetime statistics is unaffordable. Here we take 4000, which is slightly arbitrary but reasonable since it is close to usual observation time in laboratory experiments and should be able to give a reasonable estimate of the critical forcing. Furthermore, considering the small sample size and limited observation time, which is due to the cost of simulations at high Reynolds numbers, I search for the interval in $\beta$ that corresponds to relaminarisation rate of $(20 \%, 80 \%)$ at each Reynolds number and consider this interval in $\beta$ as the critical 'amplitudes'. The dependence of the critical forcing amplitude on Reynolds number is depicted in Fig. 4-8, A clear trend is seen: the larger the Reynolds number is, the larger the forcing amplitude necessary to relaminarise the flow, meaning it takes higher energy input to eliminate stronger turbulence. Although the trend is expected, relaminarisation remains possible at all Reynolds numbers investigated. The highest $R e$ tested was $R e=25000$ and given sufficiently strong force $(\beta=0.48)$ turbulence still decays. Note that the $\beta_{c}$ must saturate at the mean velocity $U=0.5$ when $R e$ approaches infinity, but this does not mean the actual energy input is going to saturate. One should keep in mind that dimensionally speaking, both the centerline velocity drop and the energy input keep increasing with the increase of the mean velocity, which defines the Reynolds number $R e$. With further increase in $R e, \beta_{c}$ asymptotically approaches 0.5 (dimensionally the mean velocity $U$ ) because of the choice of the cosh-shaped target profile, which limits the centreline velocity to be above $U$. In the limit of $\beta \rightarrow U$, the velocity profile 
is a flat plug-like one. All shear is pushed towards the wall and confined within an infinitely thin layer, in which no vortices can exist. Streamwise vortices near the wall can no longer generate streaks since there is no velocity difference in the mean flow, leading to the complete breakdown of the turbulence self-sustaining process. Obviously, under this extreme circumstance turbulence resembles shear-free turbulence which eventually decays due to energy dissipation and the lack of an energy supply. In other words, turbulent pipe flows are bound to relaminarise in the absence of a mean shear in the core region.

Different target profiles, such as plug flows approximated by polynomials, can be chosen. Hof et al. (2010) managed to relaminarise localized turbulence in pipe flow with a force that locally flattened the profile to a polynomial.

\subsubsection{Linear mechanism interpretation: transient growth}

Although the full turbulence self-sustaining cycle is a nonlinear process, linear mechanisms play very important roles in the cycle (Jimenez, 2013; Kim \& Lim, 2003; Schoppa \& Hussain, 2002). In pipe flow, despite the linear stability of the basic laminar flow, infinitesimal disturbances can be transiently amplified before the viscous decay because of the nonnormality of the normal modes (Trefethen et al., 1993; Reddy \& Henningson, 1993; Grossmann, 2000; Meseguer \& Trefethen, 2003), the socalled transient growth. It is believed to play an essential role in streak formation and amplification. Normal mode analysis shows that transient growth is most efficient through streamwise vortices. These optimal disturbances are found to generate streaks (Meseguer \& Trefethen, 2003; Willis \& Hwang, 2010) by lifting up low speed fluid from the wall to the faster core region, and weak streamwise streaks can be greatly amplified via the transient growth. This 'lift-up' process is believed to play important roles not only in the transition to turbulence but also in the sustaining cycle of fully turbulent flow. For a review on this topic see Brandt (2014).

Linear studies show that the amplitude of streaks generated by optimal streamwise vortices scales with Reynolds number $G(R e) \propto R e$ (Meseguer \& Trefethen, 2003). The optimal growth $G(R e)$ is defined as the maximum amplification of disturbances with respect to a given basic flow about which the linearization is performed (here the forced laminar flow). Consider the amplification of a disturbance as a function of time, then

$$
G(t)=\left(\sup _{\|\boldsymbol{\kappa}(0)\| \neq 0} \frac{\|\boldsymbol{\kappa}(t)\|^{2}}{\|\boldsymbol{\kappa}(0)\|^{2}}\right)^{\frac{1}{2}}
$$


where $\boldsymbol{\kappa}(0)$ is the initial condition, $\boldsymbol{\kappa}(t)$ is the output at time $t$ and $\|\cdot\|$ is a certain energy norm. By appropriately defining this norm, the optimal growth up to time $t$ and the optimal $\boldsymbol{\kappa}(0)$ can be derived directly from a singular value decomposition (SVD) analysis of the linearized system, see Schmid \& Henningson (1994); Meseguer \& Trefethen (2003)). There exists an optimal initial disturbance that maximizes the amplification $G(t)$ over time for each individual mode. By going through all normal modes, one can find the global optimal growth $G(R e)$ and the shape of the global most amplified disturbances. The maximum $G(R e)$ usually is associated with low wave number modes.

To perform the analysis, the Navier-Stokes equations are linearized about the

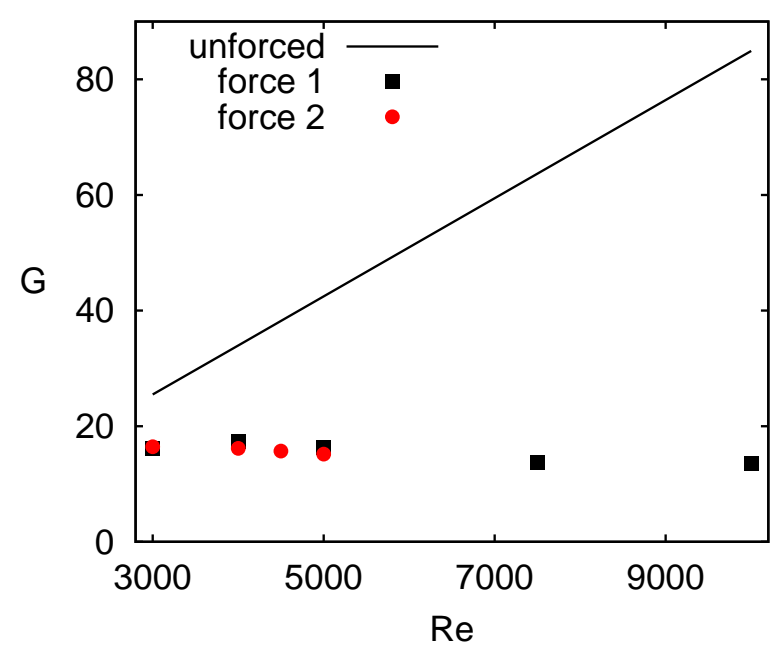

Figure 4-9: The optimal growth of the unforced basic flow (solid line) and of the critical forced basic flow (black symbols for this force and red symbols for a different type of force), i.e., the basic flow forced with critical forcing amplitudes. It should be pointed out that the intervals (uncertainty) in optimal growth corresponding to the intervals in critical forcing amplitudes shown in Fig. 4-8 are much smaller than the symbols so that can not be seen.

basic flow, usually the laminar flow. In unforced pipe flow as usual, it is just HagenPoiseuille flow. However, in a forced flow the velocity profile of the basic flow is no longer a parabola, see Eqs. (4.2). Subsequently, the optimal growth is calculated for all 'critical basic flows', namely, the laminar flows under the force with critical amplitudes $\beta_{c}$ here. For the algorithm of transient growth calculation see Meseguer \& Trefethen (2003).

Fig. 4-9 shows the critical value of $G$ as a function of Re. The optimal growth of the unforced parabolic laminar flow (the solid line) scales linearly with $R e$ in the Reynolds number range explored, agreeing well with the linear scaling $G=R e / 117.7$ 
proposed by Meseguer \& Trefethen (2003), where they investigated larger Re up to 107. In the forced basic flow (red and black symbols in Fig. 4-9) the transient growth is greatly reduced. The critical optimal growth at all Reynolds number studied here shows an almost constant value of $15 \pm 3$, much smaller than that of the parabolic basic flow. For example, at Reynolds number about $10^{4}$, the optimal growth in the absence of forcing is approximately $10^{2}$, which is an order of magnitude higher than that of the forced flow.

To test the significance of this constant transient growth at critical forcing, or in other words, to probe if turbulence requires a minimal transient growth to sustain itself, a different type of forcing was also tested, which is referred to as force 2 . This force results in velocity profiles described by a family of polynomials parameterized with the decrease in the centerline velocity if the force acts on the laminar flow. The target velocity profile in laminar flow under this force is $u_{z}=1-r^{2}-\beta\left(1-4 r^{2}+3 r^{4}\right)$. The corresponding force is determined in the same way as the force discussed before. Due to the different shape of the target velocity profiles, the critical amplitude $\beta_{c}$ is not directly comparable to the other force. Nevertheless, the resulting transient growth of the basic flow should be a fundamental mechanism and should not be dependent of the specific force. The same statistics were carried out and this force was shown to work as well as the former force, however, only up to $R e=5000$. The target profile develops inflection points when $\beta$ becomes large (above 0.25). Above $R=5000$, the $\beta$ (above 0.4 ) is large such that the inflectional instability starts to affect the flow and the force no longer can relaminarise turbulence. As a test to the influence of the time cutoff $t_{\max }$ on the statistics, for this type of force, $t_{\max }$ was chosen to be 5000 instead of 4000 as for the first force. The transient growth at the critical forcing is also shown in Fig. 4-9 (red filled circles). Surprisingly, the data agree very well with the critical transient growth under force 1 and stays approximately constant between 3000 and 5000. This result supports the argument that a constant transient growth, or streak amplification, is needed for turbulence self-sustaining. By forcing the transient growth to drop below this level, flow relaminarises and will stay laminar thanks to the linear stability.

Next we will perform yet another test of the hypothesis that turbulence requires a minimum level of transient growth. To do so we consider transient growth levels in spatially intermittent flows in the absence of any forcing. Hof et al. (2010) showed that puffs feed on the adjacent laminar profile upstream of them. If two puffs get too close to each other, the laminar profile in between will be rendered more plug-like by the upstream puff, which may kill the puff on the downstream. Indeed, 
Samanta et al. (2011) studied the interaction between a sequence of puffs separated by given distances and demonstrated that there is a minimum distance, about 40 $(20 D)$ within which the laminar profile can not fully recovered and a second puff can not be generated or survive. As shown in Sec. 1.2 this can be interpreted as analogous to the refractory length (or period) in excitable media (Hodgkin \& Huxley, 1952; Tyson \& Keener, 1988), within which the control variable can not sufficiently recover to support new excitation. Here we propose that transient growth plays the role of the control variable, and show that the minimum transient growth of the laminar flow feeding a puff is also approx 15, the same as at higher Reynolds numbers as shown in Fig. 4-9,

Fig. 4-10(a) shows a pipe flow (pipe length $L_{z}=360$ ) at $R e=2200$ globally

(a)

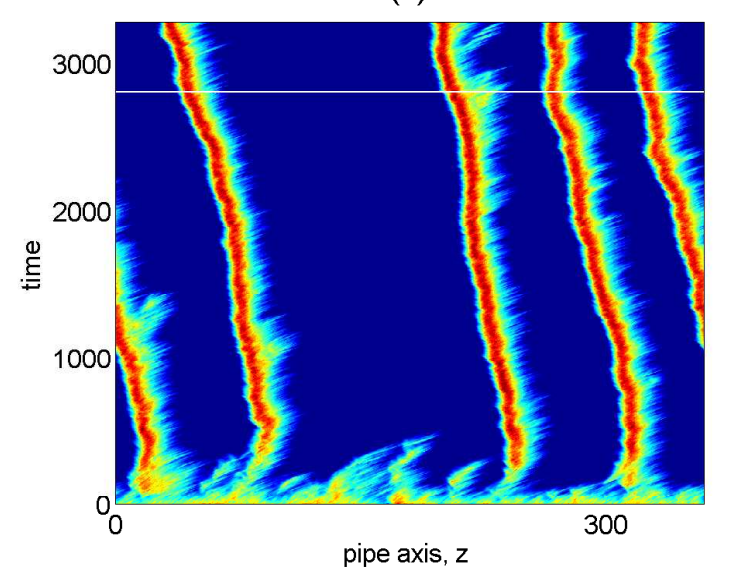

(b)
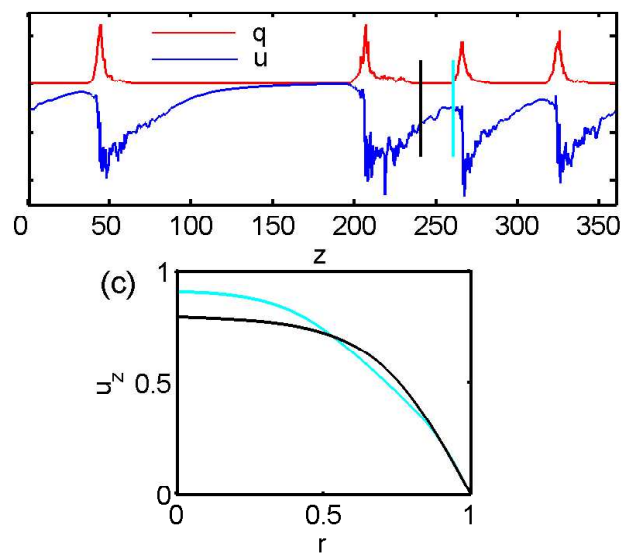

Figure 4-10: (a) A set of puffs result from global perturbations at $R e=2200$. At $t=$ 2800 (the white line), the two puffs in the middle which are separated approximately by a distance of 40 (the puff-puff interaction distance according to Samanta et al. (2011)) are investigated. (b) The local turbulence intensity $q$ and the centerline velocity $u$ at $t=2800$ are plotted along the pipe axis. The velocity profiles at two positions (marked by the black and cyan lines) are probed. The black one is within the puff-puff interaction distance and the cyan one is slightly beyond this interaction distance. (c) The velocity profiles at the two positions shown in (b).

perturbed at $t=0$. The space time plot is in a frame co-moving with the mean flow. At this Re flow is excitable (see Chapter. 3) and only can locally support localized turbulence, as evidenced by the figure. Laminar gap quickly opens up in the perturbation and 4 puffs appear and survive. The flow at $t=2800$ (marked by the white line in figure (a)) is considered since the two puffs in the middle are separated by a distance of about 40, the puff-puff interaction distance (Samanta et al., 2011). In Fig 4-10(b) the local intensity $q(z)$ and centerline velocity $u$ are plotted. Two posi- 
tions in the laminar gap between the middle two puffs are investigated, denoted by vertical black and cyan lines. The cyan one is taken right at the upstream front of the second puff from right. The velocity profiles are shown below in Fig 4-10(c) with the same color settings. As can be seen the velocity profile is relaxing to but not yet a parabola as it moves away from the upstream puff, so is the transient growth to the value for a parabola (about 19 at $R e=2200$ ). The transient growth of these two laminar profiles are 10.66 (black) and 15.32 (cyan). Since the distance between those two puffs is already the puff-puff interaction distance (i.e., the minimum possible distance), no puff can survive on the left of the cyan vertical line. In another word, also in pipe flow in the absence of any forces, no puff can survive in a laminar flow with a transient growth smaller than about 15. If viewing pipe flow in the context of excitable media, laminar flow that has a transient growth smaller than $\sim 15$ is not even excitable, at any Reynolds number.

This study demonstrates that, surprisingly a linear argument, i.e., the transient growth, sets a threshold for pipe flow turbulence to be sustained (for the system to become excitable).

\subsubsection{The control efficiency: energy saving}

The forcing control is a type of active control, which needs an energy input into the flow. To assess the efficiency of this forcing strategy, the energy input and the pumping energy reduction were calculated respectively. The power input is evaluated as

$$
<\boldsymbol{f} \cdot \boldsymbol{u}>=\int_{V} \boldsymbol{f} \cdot \boldsymbol{u} d V
$$

where $\boldsymbol{f}$ is the force per unit volume and $\boldsymbol{u}$ is the velocity field. On the one hand, the force tends to increase the wall friction drag because the force tends to accelerate the flow near the wall and generate a higher velocity gradient than the unforced turbulence. On the other hand, once the force is applied it also causes a decrease in turbulence intensity, and consequently the decrease in the driving pressure gradient and the velocity gradient at the wall. Hence, the velocity gradient at the wall, equivalently the friction drag, under the forcing may end up even lower than the unforced turbulent flow. In any case, the force itself balances a part of the friction drag, and the energy consumption due to this fact is included in the term $\langle\boldsymbol{f} \cdot \boldsymbol{u}\rangle$. In fact, only the drop in the energy (power) of the driving pressure gradient on the flow and the energy input term $\langle\boldsymbol{f} \cdot \boldsymbol{u}>$ need to be calculated. If the former outweighs the latter, an energy saving is achieved. 
(a)

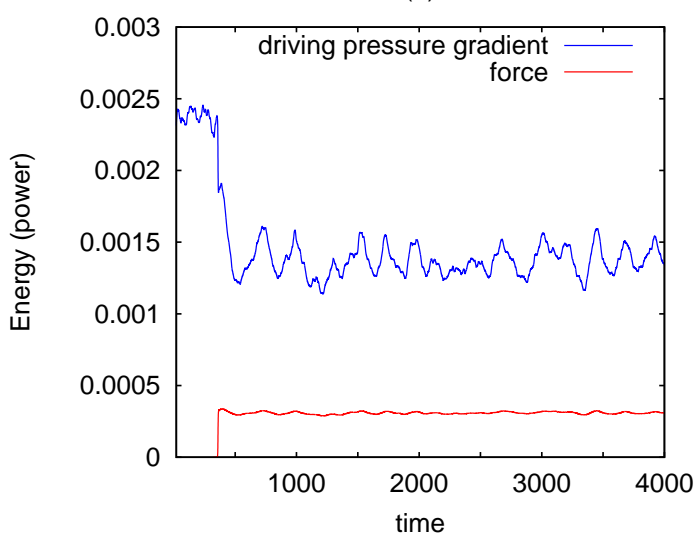

(b)

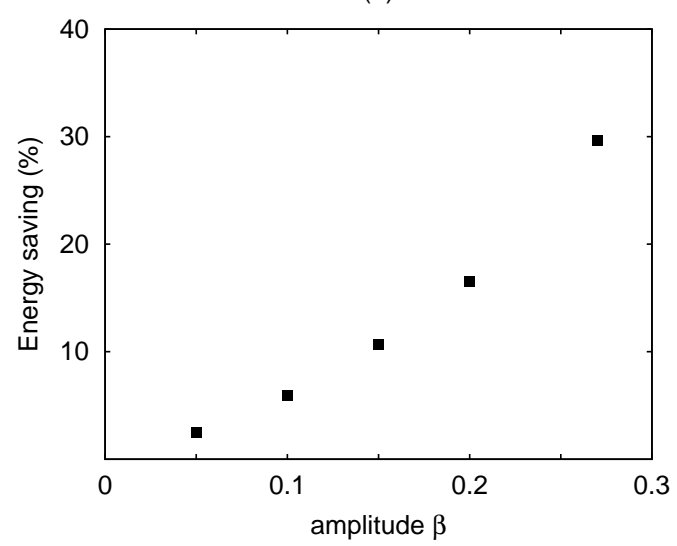

Figure 4-11: (a) The time series of the energy (power) per unit pipe length of the driving pressure gradient (blue) and of the force 2 (red) for a run at $R e=4000$ with $\beta=0.20$. (b) The net energy saving against forcing amplitude $\beta$.

Clearly, the energy input is dependent of the forcing amplitude, and the pressure gradient needed to fix the mass flux changes as the turbulence intensity changes. Fig. 4-11(a) shows the power of the driving pressure gradient and of the force from one run for the case $R e=4000 ; \beta=0.20$; force 2 . The blue line represents the power of the pressure gradient per unit pipe length, which decreases as the turbulence intensity decreases as a consequence of the activation of the force (the red line). Both the blue and red lines stabilize at a certain level after the turbulence survives and levels off under the force. The drop in the blue line is larger than the increase of the red line, as can be seen in the figure (a), giving a net energy saving even if turbulence does not completely collapse. Fig. 4-11(b) shows the energy saving for different forcing amplitudes. Surprisingly, the forcing always gives a net energy saving regardless of the amplitude. It indicates that the effect of the drop in the turbulence intensity always outweighs the tendency of increase in the wall friction due to the force. Besides, this figure shows that the energy saving increases as the amplitude increases.

The net energy saving at the critical forcing at all Reynolds numbers we considered are shown in Fig. 4-12 (b) for both forces. The symbols represent the case where turbulence survives under the force (as the case in figure (a)), and the black line represents the ultimate energy saving if the flow relaminarises and force is deactivated. The flow will stay laminar unless again strongly perturbed because of the linear stability. The results show that these two forcing strategies can achieve about $40 \%$ energy saving with the critical force amplitude for those cases where turbulence 


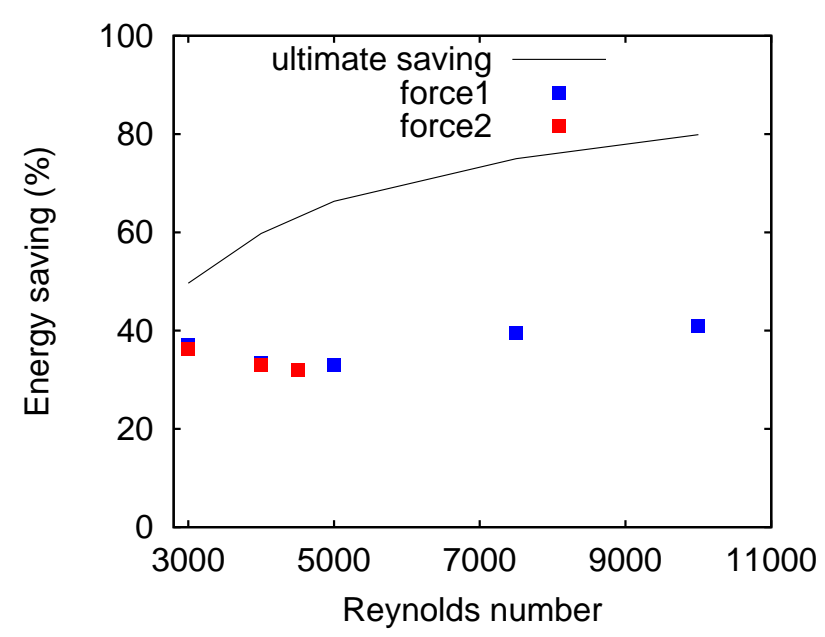

Figure 4-12: The net energy saving against Reynolds number in case of critical forcing. Symbols represent the energy saving at the critical forcing in case of turbulence survives. The black line represents the ultimate energy saving after turbulence relaminarises and force is removed.

marginally survives. It should be pointed out that, the energy saving roughly stays constant for all these cases, about $40 \%$.

\subsubsection{Localized forcing}

In experiments, global forcing would be difficult to implement. While above the forcing was applied globally throughout the pipe, in principle relaminarisation can be achieved by a local forcing. If the flow is forced fully relaminarised at one location it will remain laminar downstream owing to the linear stability of the flow. In light of the fast relaminarisation in some cases as shown in Fig. 4-6, it is inferred that turbulence can be relaminarised over a short region under sufficiently strong force. This strategy was tested at $R e=8000$ in a long pipe with an axial length of $L_{z}=360$ and the force is confined in a short region $10<z<90$. A strong force with $\beta=0.47$ is chosen here. As shown in Fig. 4-13)(a), the local turbulence intensity $q(z)$ starts decreasing once the force is activated at $t=60$ and a laminar gap (blue region) quickly opens up downstream the forcing area. Turbulence keeps coming from the upstream, it relaminarises when passing through the forcing area and stays laminar downstream (unless perturbed) because of the linear stability of the laminar pipe flow. Fig. 4-13(b) shows the change in the centreline velocity at several time instants. At $t=100$, the force has been on for 40 time units, the centreline velocity has already dropped significantly in the forcing area. As time elapses, the force keeps tearing up the turbulence as it passes by. At $t=400$ and 900, the centreline velocity downstream 
the forcing area has closely approached to the laminar value. The results here also show that it takes hundreds of radii for the parabolic profile to develop.

Note that the turbulence downstream develops a strong interface (the red stripe

(a)

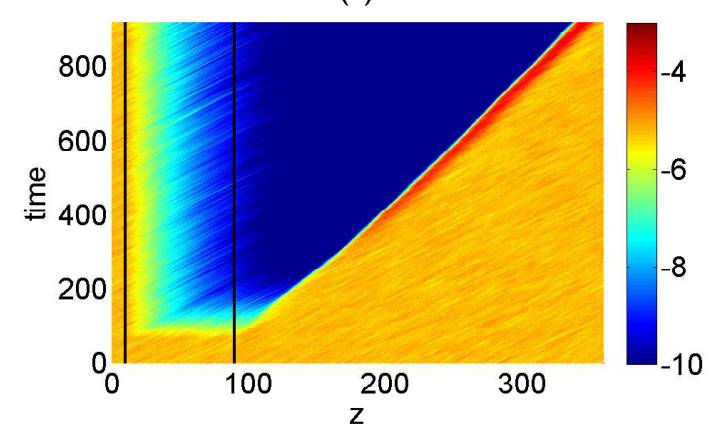

(b)

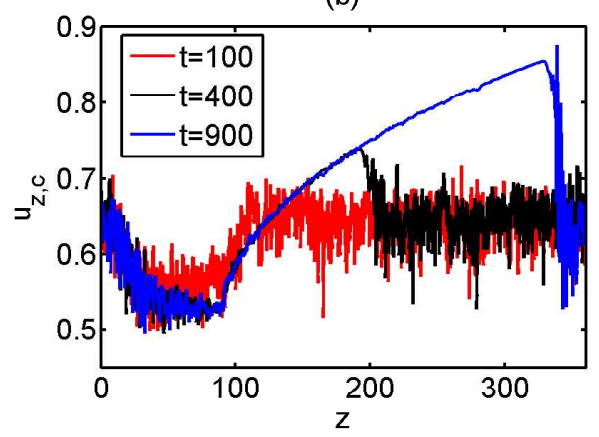

Figure 4-13: A localized control at $R e=8000$. The pipe length $L_{z}=1360$, the force $(\beta=0.47)$ is activated from $t=60$ on and is confined in the region $10<z<90$ (between the two vertical black lines in (a)). (a) The quantity $E_{c}=\iint\left(u_{r}^{2}+u_{\theta}^{2}\right) r d r d \theta$ is plotted in a logarithmic color scale with blue showing laminar region and red and brown showing turbulent region. (b) Centreline velocity $u_{z, c}$ plotted at several time instants: $t=100$ (red), 400 (black), and, 900 (blue).

in Fig. 4-13(a)) because the velocity profile immediately upstream of the turbulent flow is nearly parabolic and the vortices can extract more energy from the recovered mean shear. This is the same mechanism giving rise to the sharp upstream edge seen in puffs and slugs. Inside the forcing area, the basic flow is altered such that the linear transient growth is reduced and consequently streak formation is suppressed as discussed before. The system is rendered unexcitable (refractory) and no turbulent excitation can survive, which results in relaminarisation. Ideally, this control mechanism can keep the downstream flow laminar in an arbitrarily long pipe so that drastically reduces the friction drag with a fixed energy consumption due to the forcing, which will be much smaller than the energy saving in long pipes. This implies that relaminarisation can be achieved in experiments by locally perturbing the flow and creating a velocity profile that redistributes the mean shear as the force does here.

\subsection{Control with streamwise vortices}

The body force in Sec. 4.1 is not easy to implement in practice with non-electricconductive fluids. In this section I seek other possibilities of achieving relaminarisation. It is known that in wall-bounded turbulence, structures with different length 
scales, located at different wall distances, organize themselves so that the momentum transfer in the wall-normal direction stays in balance, resulting in a certain 'natural' velocity profile. A fixed 'unnatural' velocity profile breaks this balance and causes substantial changes in the turbulence intensity (Tuerke \& Jimenez, 2013). This implies that there may exist a way of driving turbulence far from or even out of its attraction basin by disturbing it in such a way that the perturbed flow fields (either the mean velocity profile or structures, or both) are 'unnatural'.

In Sec. 4.1 it was shown that if the velocity profile was forced unnatural (flatter than that in normal turbulence), turbulence decays. This implies that a flow field from a very high Reynolds number, which has a very flat velocity profile, may decay if $R e$ is reduced to a much lower value (still in the turbulent regime), at which turbulence naturally has a less flat velocity profile. To test this idea, a set of Reynolds number reduction tests was conducted where the velocity field from higher Reynolds numbers (above 3000) were taken as the input at $R e=3000$. These velocity field possess flatter velocity profiles and smaller structures than the normal turbulence at $R e=3000$. Relaminarisation was indeed observed if the Reynolds number drop is large enough, i.e., if the velocity field is sufficiently 'unnatural'. Statistics showed that the larger the reduction in $R e$ is, the more likely turbulence will decay. For details of these tests see Appendix A,

Turbulent pipe flow has a more plug-like mean velocity profile than that of the laminar flow because of the increased radial momentum transport via streamwise vortices. This suggests that by introducing extra streamwise vortices on top of an existing turbulent flow, the vortices will intensify the radial momentum transfer and flatten the mean velocity profile of the flow further. From the discussion in Sec. 4.1 we would expect that this equally leads to a reduction in transient growth and reduce turbulence level. We aim at designing streamwise vortices which perturb the turbulence sufficiently to lead to relaminarisation. Furthermore, experimental implementation of streamwise vortices is possible with vortex-generation techniques (Iuso et al., 2002).

\subsubsection{Configuration and generation of the vortices}

A family of two-dimensional streamwise rolls is designed. The flow field of these rolls has to comply with the incompressibility condition. For two dimensional flows, it is only needed to specify either the radial component $u_{r}$ or the azimuthal component $u_{\theta}$, the other component is readily obtained by imposing the incompressibility condition. For the spectral code and boundary conditions, it is convenient to specify the 
radial component $u_{r}$ and derive $u_{\theta}$ from the mass equation. For the first study, $u_{r}$ is chosen arbitrarily, subject to the no-penetration boundary condition at the wall. Because of the cylindrical coordinates and the accompanying singularity problem at the pipe center, the parity of $u_{r}$ as a function of $r$ has to be chosen carefully to avoid singularities. For these purposes, the following functions for $u_{r}$ is chosen:

$$
u_{r}(r, \theta)=\left\{\begin{array}{l}
\frac{\sin \left(\frac{\pi}{2} r\right)\left(1-r^{2}\right)^{2} \cos (m \theta) a}{\max _{r \in[0,1]}\left(\sin \left(\frac{\pi}{2} r\right)\left(1-r^{2}\right)^{2}\right)}, \quad m \text { is even } \\
\frac{\cos \left(\frac{\pi}{2} r\right)\left(1-r^{2}\right)^{2} \cos (m \theta) r^{2} a}{\max _{r \in[0,1]}\left(\cos \left(\frac{\pi}{2} r\right)\left(1-r^{2}\right)^{2} r^{2}\right)}, \quad m \text { is odd }
\end{array}\right.
$$

where $m$ is the azimuthal wave number, which gives the number of pairs of counterrotating vortices, and $a$ the amplitude. In both cases, the profile is normalized by its maximum for the convenience of manipulating the amplitude, namely, the amplitude is defined as the maximum of $u_{r}$ over $r \in[0,1]$. Fig,4-14(a) shows the profiles chosen for $u_{r} . u_{r}(r, \theta)$ is odd for even $m$, but even for odd $m$. The maxima of both profiles are located approximately at the center of the radii. However, it is not difficult to tune the position of the maxima. Fig. 4-14(b) shows the resulting vortices with an azimuthal wave number $m=4$, consisting of 4 pairs of counter-rotating vortices filling the pipe. The elongated structure in the radial direction enables the vortices to mix the slow and high speed flow, as explained above. With the vortices given by (4.8) we expect to control the size and positions of the structures in turbulence.

Once the configuration of the vortices is determined, the next thing to do is to generate these vortices in the numerical implementation. Here the force is an inplane force $\boldsymbol{F}(\boldsymbol{r}, \boldsymbol{\theta})$. To determine this force, one can choose the target flow field as the vortices themselves $\boldsymbol{u}(r, \theta)$, and finally derive $\boldsymbol{F}$ by putting the target flow $\boldsymbol{u}$ in Eqs. (4.1).

\subsubsection{Deforming the mean velocity profile with vortices}

Given the desired force $\boldsymbol{F}$, the flow will be driven to form the desired streamwise vortices. The effect of these vortices on fully turbulent flow is first explored at $R e=$ 5000 in a $L_{z}=4 \pi$ pipe. The force is imposed on top of the turbulence gradually until it reaches its desired amplitude, by giving a time-dependent prefactor to the force. Fig. 4-15(a) shows how this prefactor changes with time (the red line). Before the force is turned on, the flow evolves freely for about 40 time units. Then the force is 


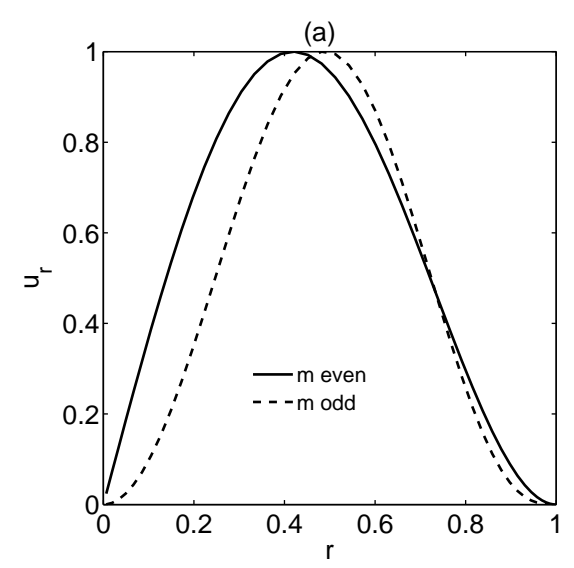

(b)

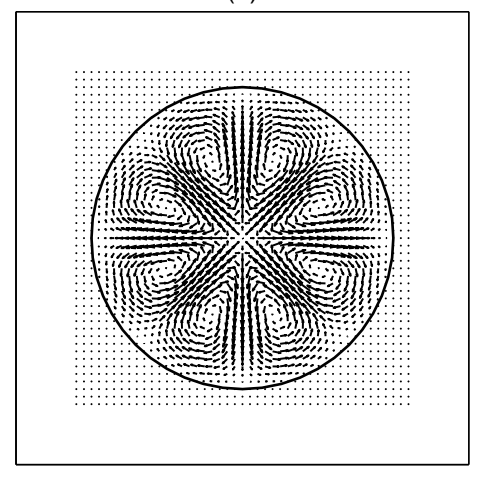

Figure 4-14: (a) Profiles of the radial velocity component $u_{r}$ of the vortices. Note that the parity of the profiles are different for different $m$, the azimuthal wave number of the vortices, to circumvent singularity at the pipe center $r=0$. (b) In-plane velocity field plotted on a cross section of the vortices with azimuthal wave number $m=4$. It looks the same at any axial position because of the absence of an axial variation (2-dimensional rolls). The magnitude of the velocity (the length of the arrow) is of no importance.

activated sharply but smoothly. As explained, the vortices are designed to deform the velocity profile into a more plug-like one. To verify this, streamwise rolls with azimuthal wave number $m=2$ and amplitude 0.05 are tested on the turbulence at $R e=5000$. Then the centreline velocity is measured and also shown in Fig. 4-15)(a). One can clearly see that it decreases by about $9 \%$ from approx 0.67 to 0.62 . The mean velocity profile before and after the forcing are displayed in Fig. 4-15(b). The velocity indeed is flattened and deformed in a similar way to the previous forcing experiment, which is a positive signal for relaminarisation. In the previous section, it was shown that with a flatter mean velocity profile, the intensity of the turbulent fluctuations tends to decrease. In the current case, a similar phenomenon is also observed (see Fig. 4-15(c)). The kinetic energy of the turbulent fluctuations $E_{k \neq 0}$ decreases by approx one third after the force is activated. We argue that this is because the force affects the mean velocity profile and the mean shear, which is crucial for the production of streaks (Hof et al., 2010; Tuerke \& Jimenez, 2013).

Streamwise rolls with different azimuthal wavenumbers and amplitudes obviously deform the flow field differently. The effects of the azimuthal wave number and amplitude are investigated separately (see Fig 4-16). On the one hand, it is found that for a given azimuthal wave number, the larger the amplitude of the vortices, the more the mean velocity profile will be deformed, as shown in Fig 4-16(a). On the other hand, for a specific amplitude of the vortices, Fig 4-16(a) indicates that the 
(a)

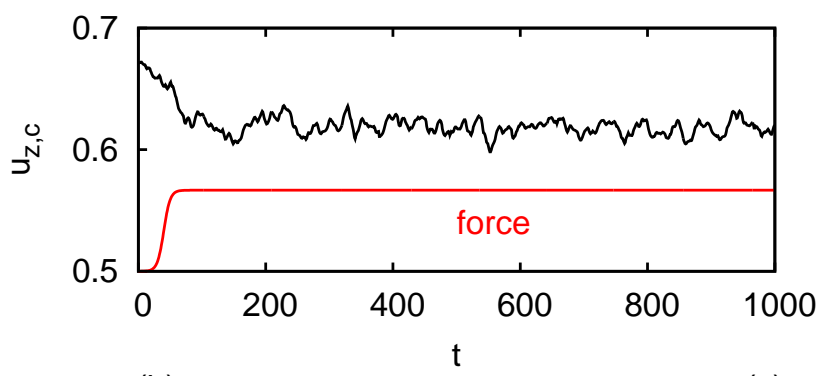

(b)
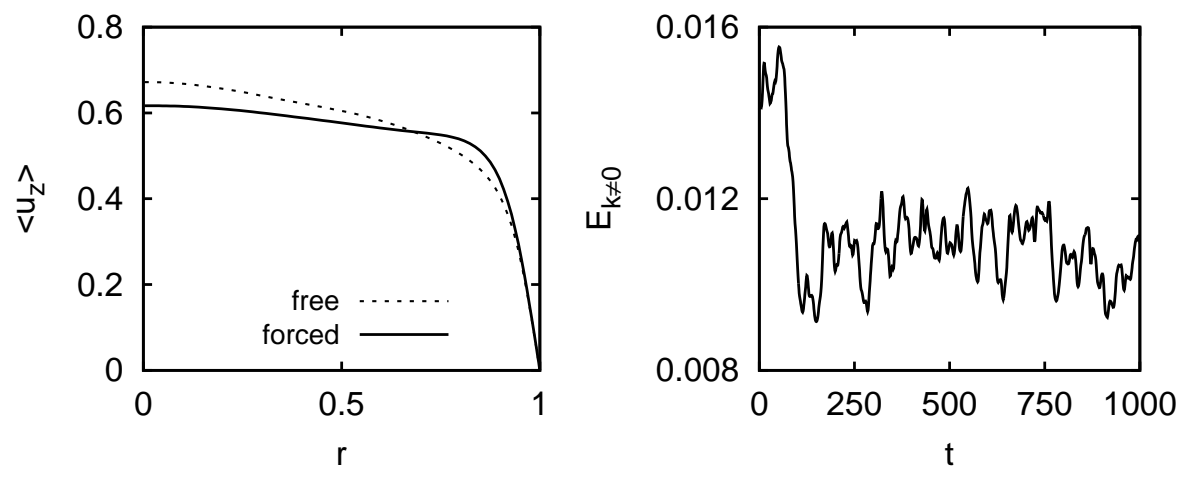

Figure 4-15: The effects on the turbulence at $R e=5000$ of the forced vortices with azimuthal wave number $m=2$ and amplitude 0.05 . (a) The realization of the force (red) and the time series of the centerline velocity (black). The force is activated at $\mathrm{t}=40$. It is turned on quickly but smoothly to its desired amplitude (normalized by the desired amplitude). (b) The comparison between the time-averaged mean velocity profile of the free turbulence and that of the forced turbulence. The dashed line represents the time-averaged mean velocity profile measured between time $t=200$ and $t=1000$. (c) The kinetic energy of the turbulent fluctuations $E_{k \neq 0}$, normalized by the kinetic energy of the Hagen-Poisseuille flow. The energy due to the force, which is axially invariant, is then excluded.

vortices with higher wavenumber affects the shape of the mean velocity profile less. At this point, it can be concluded that low-wave-number vortices are more efficient in deforming the mean velocity profile than vortices with higher wave numbers.

\subsubsection{Control with vortices at $\mathrm{Re}=3000$}

One should note that, from the point of view of turbulence control, the force should be removed after a while, otherwise it keeps disturbing the flow and is not energy efficient. The mean velocity profile is only one aspect of the flow, the detailed structures are also important. The imposed force clearly 'injects' structures into the flow, which may support the turbulence if the scales of the structures are natural to the 
(a)

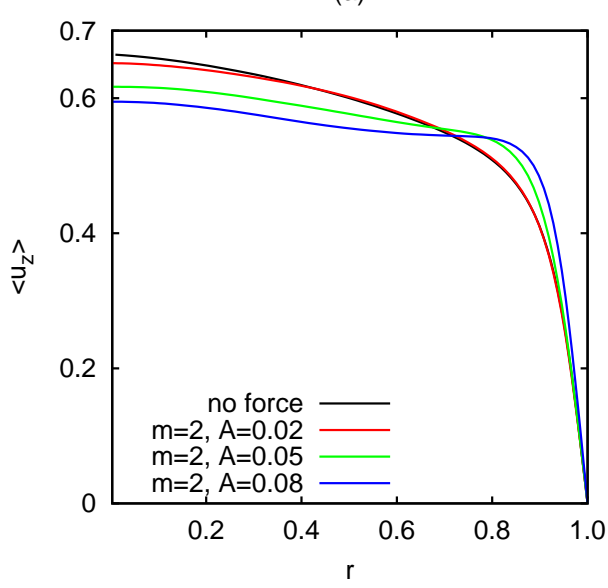

(b)

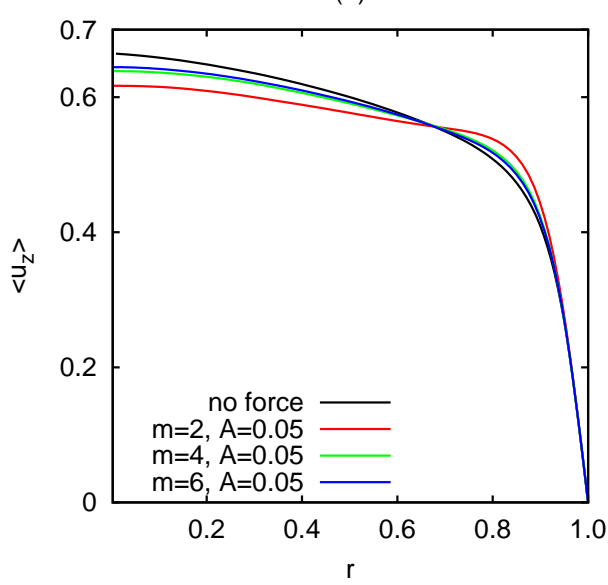

Figure 4-16: The time averaged mean velocity profile of the turbulence under the force compared to that of the free flow. (a) The azimuthal wave number of the forces (also the vortices) is fixed at $m=2$ and amplitudes $A=0.02,0.05$, and 0.08 are tested. (b) The effect of vortices with different azimuthal wave numbers but the same amplitude is tested. Wave number $m=2,4$, and 6 , and amplitude $A=0.05$ are tested.

turbulence, or counteract the turbulence if the structures are unnatural.

Two controls with streamwise rolls with azimuthal wave number $m=2$ and $m=12$ and the same amplitude $A=0.18$ are tested (see Fig. 4-17). The centreline velocity decreases from about 0.68 to 0.58 in the $m=2$ case (solid line, between $t=40$ and 100), but only to 0.65 in $m=12$ case (dashed line). However, the $m=2$ vortices fail to relaminarise the flow after the force is turned off. The turbulence intensity, here measured by the kinetic energy of the transverse velocity fluctuations $E_{c}=\int_{V}\left(u_{r}^{2}+u_{\theta}^{2}\right) d V$, recovers to the natural level at this Reynolds number (see Fig. 4-17(b), solid line). Note that during the forcing, $E_{c}$ is much higher than the natural level because the force generates $u_{r}$ and $u_{\theta}$ components and clearly injects energy into the flow. Surprisingly, the $m=12$ vortices manage to remove turbulence from the flow. The turbulence intensity decreases quickly and monotonically after the force is removed. To look into the details of this process, isosurfaces of the streamwise velocity deviation from the basic flow $\delta u_{z}= \pm 0.2$ at some instants are plotted in Fig. 4-18, The survival case (the solid line in Fig. 4-17), the $m=2$ case, is on the left. 4 snapshots are taken at $t=0,96,208$, and 400. At $t=0$, turbulence is unforced and one can see the configuration of the streaks. Approximately four high speed streaks can be seen near the wall. At $t=96$, the force has been on for 50 time units and the flow is drastically perturbed by the vortices. Four large high speed streaks are 
(a)

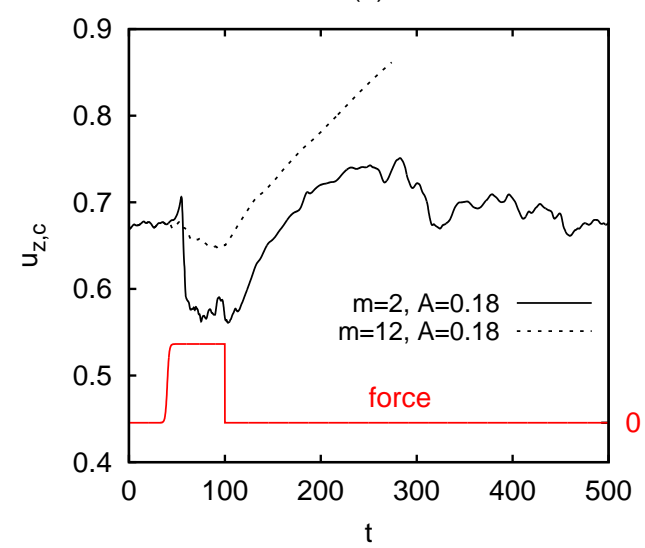

(b)

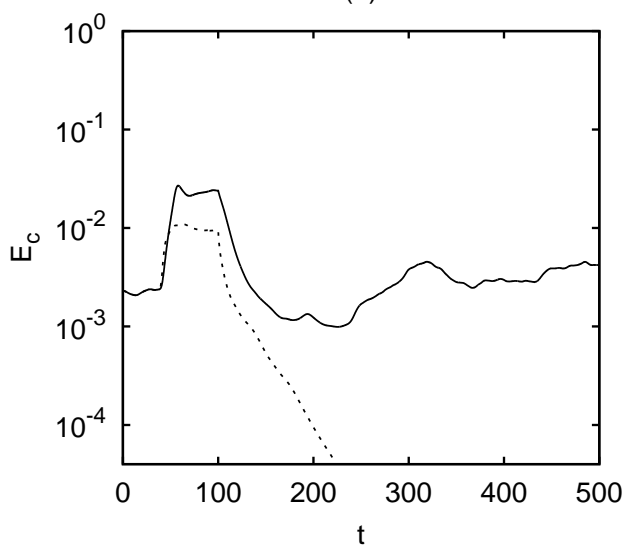

Figure 4-17: Control with vortices with azimuthal wave number $m=2$ (solid line) and $m=12$ (dashed line), and amplitude $A=0.18$ for both. The force (vortices) is activated at $t=40$ and deactivated at $t=100$. (a) Time series of the centreline velocity of mean velocity profile of these two controls. (b) Time series of the kinetic energy of the in-plane velocity fluctuations in the whole pipe, $E_{c}=\int_{V}\left(u_{r}^{2}+u_{\theta}^{2}\right) d V$.
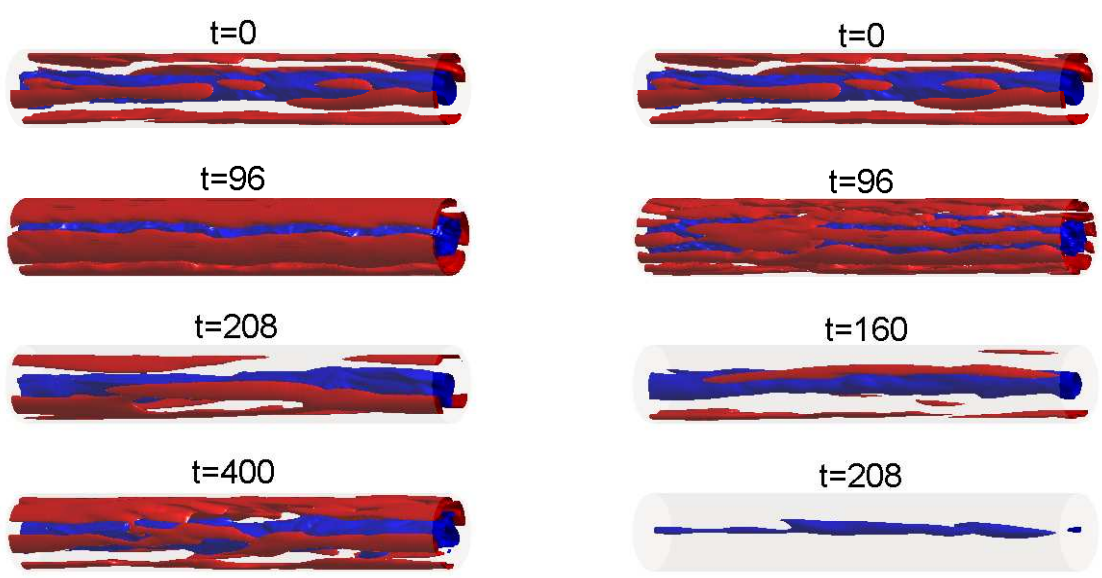

Figure 4-18: Control with vortices at $R e=3000$ with azimuthal wave numbers $m=2$ and $m=12$, starting from the same initial condition. The flow is from bottom to top and the isosurfaces of streamwise velocity deviating from the basic flow $\delta u_{z}= \pm 0.2$ are plotted. Red color represents positive value and blue represents negative value. Note that the force is activated at $t=40$ and is off after $t=100$. Left: $m=2$ (solid line in Fig. 4-17), snapshots are taken at $t=0,96,208$, and 400. Right: $m=12$ (dashed line in Fig. 4-17), snapshots are taken at $t=0,96,160$, and 208.

formed by four large vortices. It is worth noting that the wave number of the energy containing structures in turbulence at $R e=3000$ is approximately in the range $(2,7)$ 
and peaks at $m=4$, which embraces the wave number of the introduced vortices, as shown in the energy spectra in Fig. 4-19, At $t=208$ the force is off and the turbulence intensity almost reaches its minimum, but only slightly lower than unforced turbulence (see Fig 4-17(b)). Quickly the turbulence recovers to its natural level. On the right is the $m=12$ case. During the control period, the original turbulence structures are broken down into smaller structures by the high-wave-number vortices, which is obvious in the $t=96$ snapshot. At this moment there are much more fine high speed streaks filling the pipe. After $t=100$, force is off and the turbulence decays quickly and at $t=208$ the turbulence almost relaminarises. We argue that this is because with low-wave-number vortices, in this case $m=2$, the forced large streaks have similar wave number to the original energy containing structures, so that they will support turbulence after the force is removed. However, high wave number structures created by the high-wave-number vortices are more likely to dissipate out rather than to form larger structures that favour turbulence. This is consistent with the observation made in the Reynolds number reduction section, where the initial conditions drawn from Reynolds number much higher than 3000 contains structures that do not favour turbulence at $R e=3000$, so that cause relaminarisation.

Looking at it from a different perspective, the introduced vortices changed the

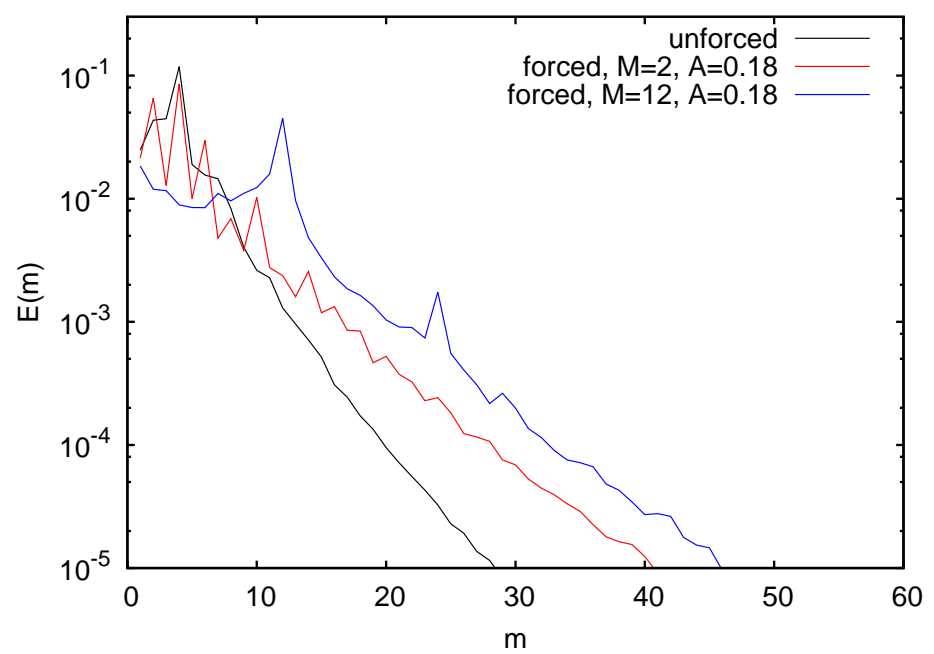

Figure 4-19: Energy spectra of the uncontrolled flow (black), of the flow controlled with vortices with wave number $m=2$ (red) and $m=12$ (blue). The x-axis is azimuthal wave number $m$ and the y-axis is the energy associated with $m$

energy spectra, or in other words, redistributed the energy over structures with different sizes. This is evidenced by Fig. 4-19, Based on the energy spectra of the uncontrolled flow, it is clear that the energy containing structures are of azimuthal 
wave number $m \leq 7$, which account for about $90 \%$ of the total kinetic energy of turbulence at this Re. The control drastically changes the energy spectra in the $m=12$ case, the original peak at $m=4$ disappears and and a new peak forms at $m=12$ because the forcing is exciting this mode. Under control, the energy spectra suggests that energy is redistributed such that the original energy containing structures are greatly suppressed by the vortices. A great amount of energy is shifted to structures with wave number around $m=12$, far way from the energy containing structures. After the control is removed, this amount energy will be transfered downwards to smaller structures and get dissipated rather than go upward back to large structures. On the contrary, in the $m=2$ case the energy spectra does not change much so that turbulence recovers after the control stops.

The same vortices are imposed in more runs to test efficiency. A stochastic behavior is also observed. With parameters $m=12$ and $A=0.18$, the turbulence relaminarises in 9 out of 20 runs. Based on the logic described above, it should be more efficient to control with streamwise vortices of even higher azimuthal wavenumbers or higher amplitude. First, a higher amplitude enable the vortices to flatten the mean velocity profile more. Second, higher wave number vortices generate smaller structures which will alter the flow field more drastically, pushing the flow farther from the natural turbulence. Once the flow is freed from the force, a flatter velocity profile and smaller structures are more likely to dissipate out. Indeed, if the wave number increases, relaminarisation occurs in 14 out of 20 runs with $m=16, A=0.18$ vortices. If the amplitude is increased to 0.22 , relaminarisation is achieved in 13 out of 20 runs with $m=12$ vortices, and in 19 out of 20 runs with $m=16$ vortices.

This process of force on being turned on for a short time $(15 D / U$ here) and then turned off is a good approximation of the process of a flow passing a segment of a pipe where turbulence is persistently perturbed. This implies the possibility of relaminarising turbulence in a long pipe with vortices-control confined in a short segment of the pipe. It is worth mentioning that this result is astonishing because one can drive a turbulent flow field out of the attraction basin of turbulence by stirring it.

\subsection{Discussion}

Following the study of transition to fully turbulent flow in Chapter 3, here I explored control mechanisms that force inverse transition, i.e., to relaminarise fully turbulent flow. 
First, I extended the forcing strategy adopted by Hof et al. (2010) to much higher Reynolds numbers. Fully turbulent flow up to $R e=25000$ was relaminarised by the force. Two types of forces were tested and proven equally effective, suggesting that the details of the forcing are not crucial for the relaminarisation. I carried out the critical forcing statistics in light of the stochastic behavior of turbulence under the forcing. In an effort to interpret the mechanism underlying the decay of turbulence, I calculated the transient growth of the forced basic flow and compared it to that of the unforced basic flow. The results show that the force greatly reduces the transient growth, which despite being a linear mechanism, is essential for turbulence to be sustained. This is consistent with the claim that transient growth is essential for the turbulence self-sustaining mechanism in fully developed turbulence (Schoppa \& Hussain, 2002). It is worth mentioning that the results (Fig. 4-9) showed that the transient growth of the 'critically' forced flow stays roughly constant at about $15 \pm 3$ all across the $R e$ range explored here. This value was also found to be the minimum for puffs to survive in unforced flows. Therefore, we proposed that the transient growth of 15 sets a boundary between excitable and refractory. This also suggests that a universal minimum streak amplification is necessary to sustain pipe-flow turbulence at all $R e$. By pushing the transient growth below this value with certain control techniques, the energy amplification can be suppressed and the flow relaminarises. Under the force, the mean velocity profile of turbulence is also greatly altered, which results in a great change in turbulence intensity (see Fig. 4-6). It is consistent with the finding of Jimenez (2013) that a change in the mean shear will break the energy balance and create a different turbulence intensity. Our results also showed that under a different velocity profile, not only the amplitude of disturbances changes a lot, but also the flow structures (see Fig. 4-5).

This critical forcing amplitude, which is measured by the centreline velocity decrease on the basic flow, may provide experiments with some information on how control techniques should be designed. Unfortunately, global body force implementation is practically impossible in experiments. I further showed that only localized forcing is necessary for relaminarising turbulence, and successfully relamianrised turbulence with a localized force up to $R e=8000$. This localized forcing can be applied in experiments by using control methods that locally modify the velocity profile in a similar way.

Second, a streamwise vortices (rolls) control method is developed with which fully turbulent flow at $R e=3000$ is relaminarised. In addition, the control duration is short, which means low energy consumption. The simulation justifies that by per- 
turbing the velocity profile, as well as the sizes, positions, and spacing of the near wall streamwise vortices and streaks properly, one can create a flow field that is out of the attraction basin of turbulence and realize relaminarisation. This is in line with the observation of Tuerke \& Jimenez (2013), who showed that unnatural velocity profiles result in abnormal turbulent intensities and structures. However, Tuerke \& Jimenez (2013) did not report relaminarisation. A possible link may be made between this result and the transient growth argument in Sec. 4.1. Here the high wave number vortices broke the flow into small structures, which are far smaller than the optimal perturbation (usually of low wave numbers, see Schmid \& Henningson, 1994; Meseguer \& Trefethen, 2003; Willis \& Hwang, 2010). This suppressed the energy growth in the unnatural flow field and caused relaminarisation. In addition, analysis into the energy spectra shows that the control greatly alters the energy spectra if the flow is controlled with vortices of an azimuthal wave number far away from that of the energy containing structures in normal turbulence. The introduced vortices act as to suppress low wave number structures and excite high wave number structures. The latter are likely to dissipate after the control is deactivated.

Large scale vortex control can be implemented experimentally by injection from the wall (Iuso et al., 2002). Iuso et al. (2002); Schoppa \& Hussain (1998) showed that vortices can reduce the wall drag. However, they did not report relaminarisation of turbulence. In their experiment/simulation, the control has to be kept on forever in order to achieve a persistent drag reduction. Our control is a large scale technique that may be implemented experimentally also by injection (and extraction if necessary). Besides, it is strong but short perturbation to the flow which accomplishes drag reduction only after the force is off and the flow relaminarises. However, the high azimuthal wave number is a challenge to experimental implementation. The optimal configuration of the vortex-control method needs to be further investigated in future studies. 


\section{Chapter 5}

\section{SLIP BOUNDARY CONDITION}

\subsection{Introduction}

Besides controls that act directly on flows, an alternative is to control the boundary conditions. The mean shear is largely determined by the boundary condition, implying that modifications of the mean shear can be achieved by manipulating the boundary condition. On macroscopic scale, viscous flow tends to stick to the wall on smooth hydrophilic surfaces, resulting in no-slip boundary condition, which is well accepted and widely used in fluid dynamics. However, on hydrophobic surfaces or finely textured surfaces, especially in microfluidics, considerable slippage or effective slippage can take place on the liquid-solid interface (Vinogradova, 1999; Cottin-Bizonne et al., 2002; Zhu \& Granick, 2002; Joly et al., 2006; Cottin-Bizonne et al., 2005; ; Joseph et al., 2006; Qu \& Rothstein, 2005). The far-field effects of the hydrophobicity or fluctuating surfaces can be modelled by an "effective partial slip" applied on smooth surfaces (Vinogradova, 1999; Stone et al., 2004; Bazant \& Vinogradova, 2008; Kamrin et al., 2011). Drag reduction has been observed on hydrophobic and patterned surfaces in both laminar flow and turbulence thanks to this slippage on the wall (Min \& Kim, 2004; Qu \& Rothstein, 2005; Jung \& Bhushan, 2010; Jovanovic et al., 2011). Slip length was usually reported to be on nanometer scale (Cottin-Bizonne et al., 2002; Zhu \& Granick, 2002; Cottin-Bizonne et al., 2005; Jolv et al., 2006) or micrometer scale in extreme conditions (Joseph et al., 2006; Qu \& Rothstein, 2005). These slip lengths are small compared to macroscopic length scales, so that the resulting slip velocity on the wall, and consequently the reduction in the mean shear are far from being sufficient to affect the turbulence self-sustaining process significantly in large devices (Min \& Kim, 2004). Recently, with the technique of surfaces texturing with 
the micro-nano hierachical structures a much larger slip length up to $400 \mu \mathrm{m}$ has been achieved (Lee et al., 2008; Lee \& Kim, 2009), which may be large enough to considerably affect flow systems even on macroscale. Park et al. (2013) pointed out that the drag reduction in laminar flow is directly due to the slip, so that the drag reduction is independent of the Reynolds number. They numerically investigated the effect of textured surfaces with microgrates and trapped air pockets on turbulent dynamics and claimed that the drag reduction is not a direct result of the velocity slip, but mainly because the slip affects the turbulence structures, which in turn results in a reduced skin friction. They also reported that above a slip length about 40 viscous unit the drag reduction effect diminished. To our knowledge, there is no report of relaminarisation of fully turbulent flow due to this slippage on the wall. In the following we apply an effective slip boundary condition to normal pipes and go far beyond the slip length that current technologies can achieve, to study what slip length is needed to relaminarise turbulence and explore possible turbulence control methods.

\subsection{Methods}

We solve the nondimensional incompressible Navier-Stokes equations

$$
\frac{\partial \boldsymbol{u}}{\partial t}+\boldsymbol{u} \cdot \boldsymbol{\nabla} \boldsymbol{u}=-\nabla p+\frac{1}{R e} \Delta \boldsymbol{u}, \boldsymbol{\nabla} \cdot \boldsymbol{u}=0
$$

in cylindrical coordinates $(r, \theta, z)$ in a straight pipe with Navier slip boundary condition (Stone et al., 2004) on the wall for axial velocity

$$
\left.\left(\lambda \partial u_{z}+u_{z}\right)\right|_{r=1}=0
$$

and no-slip boundary condition for azimuthal and radial velocities, $u_{\theta}(1, \theta, z, t)=0$ and $u_{r}(1, \theta, z, t)=0$, where $\lambda$ is called Navier slip length. DNS Code 2 is employed for this study since it allows easier manipulation on the slip boundary condition than Code 1. Generally speaking, effective slip boundary condition in three dimensional case has been proposed to appear in tensorial form Stone et al. (2004); Bazant \& Vinogradova (2008); Kamrin et al. (2011). Nevertheless, here the slip boundary condition is only imposed on $u_{z}$ because 1 ) we only aim at affecting the turbulence self-sustaining process by modifying the mean shear and $u_{z}$ determines the mean shear. The simple boundary condition employed here, which raises no difficulty in numerical implementation, very well satisfies this need; 2) the slip in azimuthal di- 
rection actually tends to increase turbulence intensity (see Fig. 5-1), which is hence unfavourable in the context of turbulence control; 3) potentially for comparison to ongoing control experiment in our group where one pipe segment is moved in the streamwise direction which generate streamwise velocity at the wall. In fact, as shown later, the streamwise slip dominates the flow in case of slip in both azimuthal and streamwise directions is imposed. The periodic boundary condition is imposed in the axial direction, hence, a Fourier-Fourier-finite difference scheme is employed for the spatial discretization. The equations are integrated in time with a $2^{\text {nd }}$ order semiimplicit scheme and the incompressibility is imposed using a time-splitting scheme, while the mass flux is kept constant (see Chapter 2 for details).

\section{$5.3 \quad$ Results}

With the forementioned partial slip boundary conditions, normalization, and constant mass flux, the velocity profile of the laminar flow can be derived analytically as $\boldsymbol{u}=\left(1-r^{2}+2 \lambda\right) /(1+4 \lambda) \hat{\boldsymbol{z}}$, the corresponding mean pressure gradient $\boldsymbol{\nabla} p=-4 / \operatorname{Re}(1+4 \lambda) \hat{\boldsymbol{z}}$, and the velocity slip on pipe wall $\boldsymbol{u}_{w}=2 \lambda /(1+4 \lambda) \hat{\boldsymbol{z}}$. Therefore, $\lambda=0$ corresponds to no-slip condition $u_{z}=0$, recovering the Hagen-Poiseuille flow, and $\lambda=\infty$ gives full slip condition $\partial u_{z} / \partial r=0$ under which the velocity profile is completely flat, $u_{z}(r)=\frac{1}{2}$, as in inviscid pipe flow. In turbulent flow, these quantities fluctuate and can only be determined by numerically solving the governing equations.

The influence of the azimuthal slip is first explored. Intuitively, the azimuthal slip favours streamwise vortices, which are then more efficiently creating streaks. Therefore, azimuthal slip will intensify turbulence, so that is unfavourable in the context of turbulence control. This is actually observed in our simulation, as illustrated in Fig. 5-1. A comparison between a turbulent flow with only azimuthal slip boundary condition $\lambda \partial u_{\theta} / \partial r+u_{\theta}=0$ with $\lambda=0.22$ and one with no-slip condition is made at $R e=7500$. The kinetic energy of the in-plane velocity components of the turbulence, $E_{c}=\frac{1}{2} \int_{V}\left(u_{r}^{2}+u_{\theta}^{2}\right) d V$, is depicted in Fig. 5-1(a). Once the azimuthal slip is turned on, $E_{c}$ experiences a sharp increase and then stabilizes on a level twice as high as that with no-slip boundary condition. Fig. 5-1(b) compares the contours of streamwise vorticity in a cross section in each case, an intensification of the vorticity in near wall regions due to the azimuthal slip is apparent. Similar effects were also observed in channel flow (Min \& Kim, 2004). Imposing slip in both directions however leads 


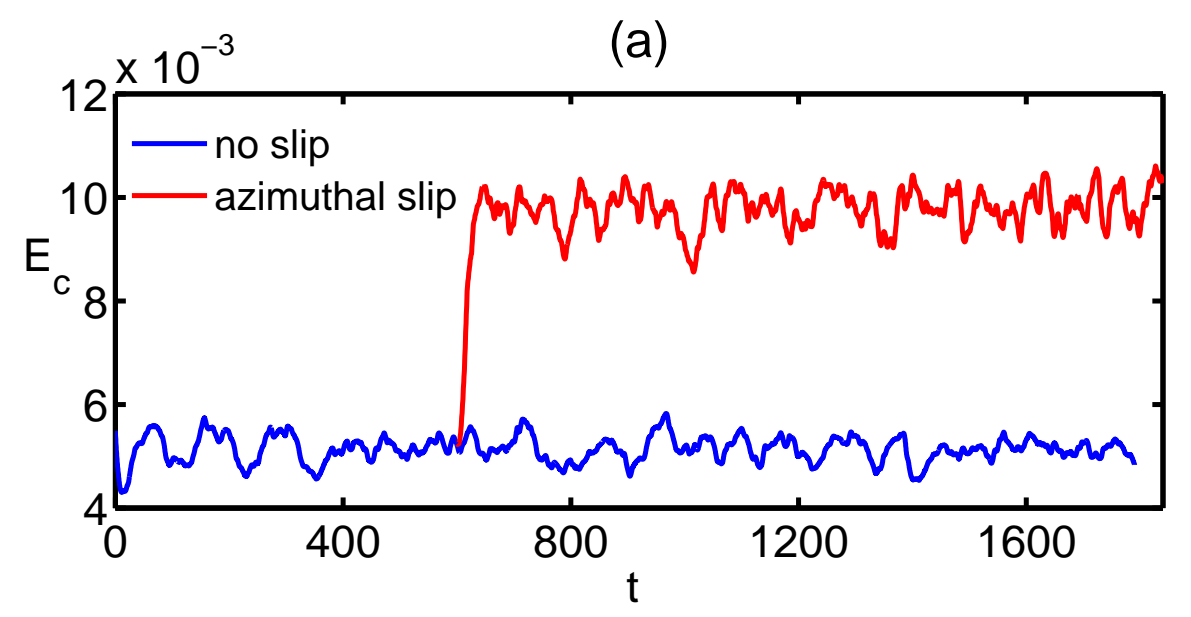

(b)

(c)
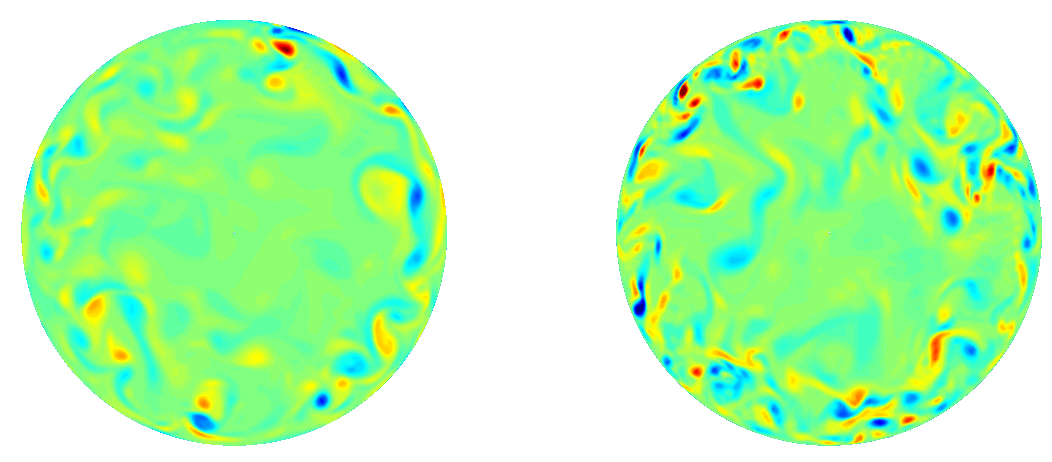

Figure 5-1: Azimuthal slip intensifies turbulence. Simulations conducted at $R e=$ 7500. (top) Time series of kinetic energy of the transverse flow $E_{c}$ normalized by the energy of Hagen-Poisseuille flow for two turbulent flows: one with no-slip boundary condition (blue) and the other with azimuthal slip condition with slip length $\lambda=0.22$ (red). In the latter, the azimuthal slip is turned on at $\mathrm{t}=600$; (bottom) contour of streamwise vorticity $\omega_{z}$ plotted on the cross section at the axial position where $\iint \omega_{z}^{2} r d r d \theta$ takes the maximum: (b) the flow with no-slip boundary condition, and (c) the flow with azimuthal slip boundary condition. Note that red and blue region represents strongly vortical regions and both of (b) and (c) are taken at $t=700$ from the time series shown in (a).

to an overall reduction of turbulence, and the streamwise slip totally dominates the flow (see Fig. 5-2). Since this study is partially motivated by experiments where relaminarisation was achieved by moving pipe segments in the streamwise direction, here we will only focus on the effect of slip in the streamwise direction.

We initiate our study with fully turbulent flow simulation with no-slip boundary condition at various Reynolds numbers ranging from 3000 to 20000. At each Re, the resolution is ensured high enough by requiring the time averaging friction coefficient 


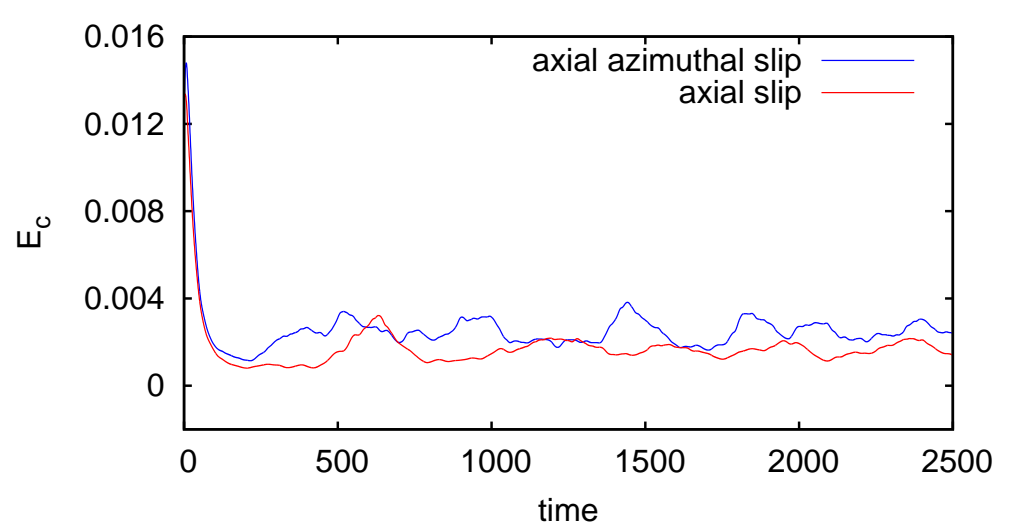

Figure 5-2: Time series of the kinetic energy of the transverse flow $E_{c}$ normalized by the energy of Hagen-Poisseuille flow for a run at $R e=7500$, with both azimuthal and axial slip length $\lambda=0.22$ (the blue line), and with only axial slip length $\lambda=0.22$ (the red line). Azimuthal slip tends to intensify turbulence, however, this figure shows the dominance of the axial velocity slip.

to agree well with the Blasius law and no energy accumulation at high wave number modes. The simulations are carried out in a $8 \pi$-long pipe at $R e=3000$ and 5000, and the pipe length is reduced to $4 \pi$ at all higher Reynolds numbers considering the enormous computing resources needed. At these Reynolds numbers for usual noslip boundary condition turbulence is well self-sustained. Subsequently, we increase the slip and study how the original turbulence reacts to the new boundary condition. Turbulence either quickly adjusts to the new boundary condition or relaminarises, depending on whether the reduced mean shear can feed it or not. This process roughly mimics the situation where turbulence flows through a pipe part of which allows slip on the wall. If the turbulence relaminarises during passing by, the flow will stay laminar forever downstream because of the linear stability of the laminar flow.

A set of runs starting from different initial conditions at the point $(R e, \lambda)=$ (7500, 0.22) are shown in Fig. 5-3. Upon turning on the slip at $t=0$, the turbulence intensity undergoes a sharp decrease, which is expected since the mean shear is reduced sharply by the slip on the wall. As mentioned before, the mean shear is the main energy source for the turbulence sustenance, the decrease in the mean shear reduces the energy that the streamwise vortices can extract to form streaks, hence weakens the streaks. In turn, weaker streaks generate weaker streamwise vortices. It turns out that with sufficiently large slip length turbulence will not be able to sustain itself and finally relaminarises. However, given marginal slip length, as the one used in Fig. 5-3, turbulence reacts in a probabilistic manner, i.e., in some runs it completely decays, but in some others manages to recover from the initial decaying 


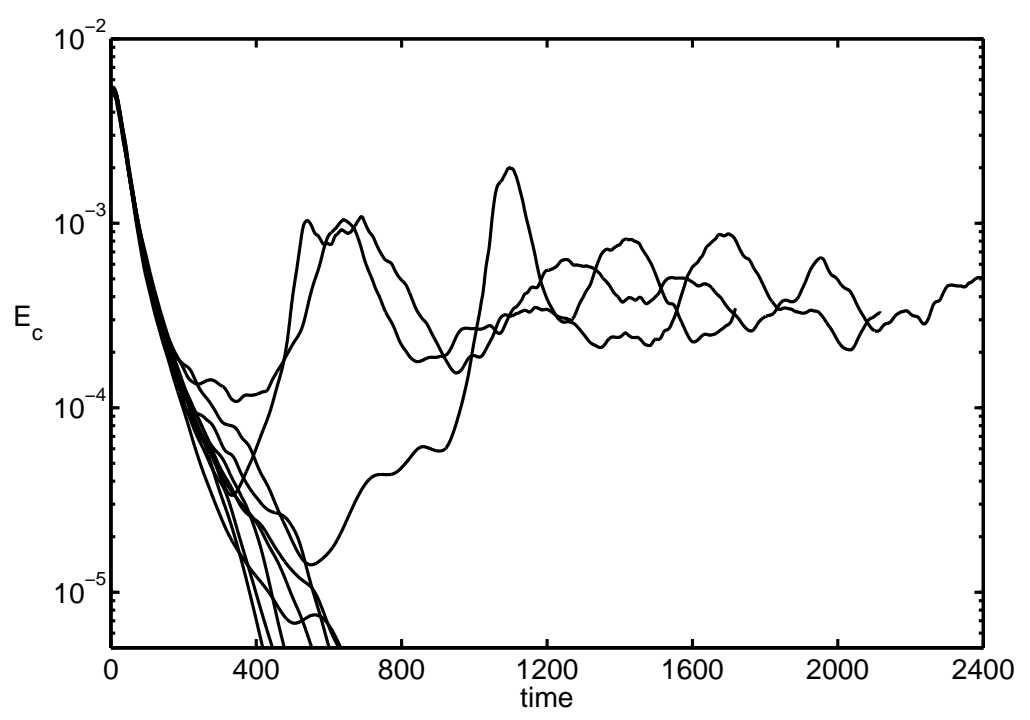

Figure 5-3: Time series of kinetic energy of the transverse flow normalized by the energy of Hagen-Poisseuille flow for 10 different runs at $R e=7500$, with a marginal slip length $\lambda=0.22$. 7 relaminarisations are observed.

and eventually stabilizes at a lower energy level than the original turbulence which does not slip on the wall, as depicted by the energy trajectories of the transverse fluctuations in Fig. 5-3. It is likely that the surviving cases would eventually also decay, however, lifetimes just like in ordinary pipe flow around $R e \sim 2000$ can be extremely long. To keep computational cost manageable we used a time cutoff $t_{\max }=4000$ and define the critical slip length as $\lambda_{c}$, where turbulence in $50 \%$ runs decays.

A statistical study is conducted on the dependence of the critical slip length $\lambda_{c}$ on Reynolds number, which is illustrated in Fig. 5-3. With such a criterion, extensive DNS studies are carried out at $R e=3000,5000,7500,10000,15000$, and 20000. At each $R e$, the $\lambda_{c}$ is searched with a bisection method with about 50-100 realisations and for each parameters combination $(R e, \lambda), 10$ runs starting with uncorrelated initial conditions are conducted. The small size of the sample is a compromise of the expensive computation due to the high resolutions needed to fully resolve the turbulence at such high Reynolds numbers. To compensate the small sample size, we extrapolate $\lambda_{c}$ for the $50 \%$ relaminarisation rate from all $\lambda$ values that gives relaminarisation rates in the interval $30 \%$ to $70 \%$. Surprisingly, the dependence of $\lambda_{c}$ on Re appears to be linear, as shown in Fig. 5-4(a). A linear fitting $\lambda_{c}=a R e+b$ gives $a=1.8752 \times 10^{-5}$ and $b=-0.037622$, resulting in an intercept on the Re-axis of about 2000. This intercept represents the maximum $R e$ at which turbulence will naturally relaminarise without any slip on the wall, i.e., $R e_{c r}$, which considering statistical uncertainties and 

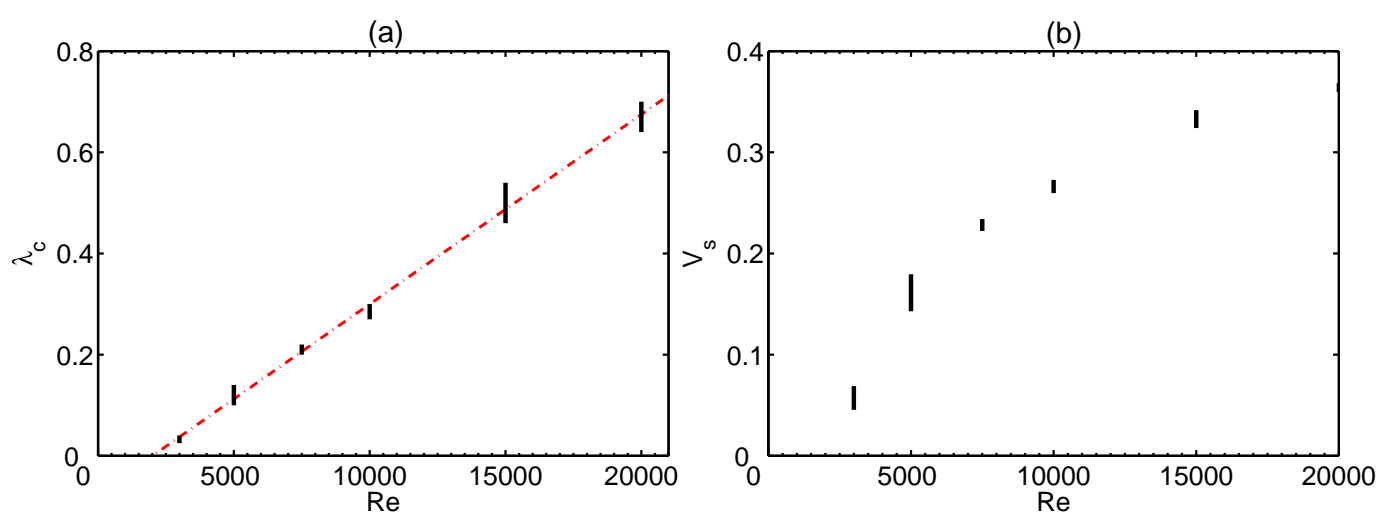

Figure 5-4: (a) The critical slip length $\lambda_{c}$ versus Reynolds number. The red dashed line is a linear fitting $\lambda_{c}=a R e+b$ using the middle points of those bars which presumably lead to about 50\% relaminarisation rate; (b) The slip velocity on the wall resulting from the slip lenghts shown in (a).

limited sample size is in very good agreement with the critical $R e_{c r}=2040$ proposed by Avila et al. (2011). Fig. 5-4(b) depicts the critical slip velocity on the wall in turbulence resulting from the critical slip lengths. The other way round, turbulence is likely to relaminarise in the presence of these amounts of velocity slip on the wall. This suggests that instead of reaching the currently unachievable huge slip length on the wall, turbulence may be relaminarised by moving pipe segments or a foil covering the pipe wall in a segment in the stream wise direction. Such experiments have indeed been carried out successfully in our group, and publication is in preparation.

\subsection{Discussion}

The relaminarisation of pipe flow turbulence under partial slip boundary condition was studied. The effect of both azimuthal slip and streamwise slip were investigated and the results show that the former tends to strengthens turbulence and on the contrary the latter weakens turbulence. Similar effect is also found in Min \& Kim (2004); Park et al. (2013). The streamwise velocity dominates the mean shear, consequently a streamwise velocity slip significantly affects the turbulence self-sustaining process in which shear play a role as the energy source. However, on normal superhydrophobic surfaces the effective slip length is very small (below a micron) and the resulting velocity slip is not enough to significantly affect the turbulence self-sustaining process, though drag reduction indeed was achieved. The DNS result shown in this chapter indicates that to relaminarise turbulence, slip lengths comparable to the pipe radius (see Fig. 5-4(a)) and velocity slip comparable to the mean flow speed (Fig. 5-4(b)) are 
needed. Surprisingly, a statistical study showed a linear scaling of the minimum slip length for relaminarisation with $R e$, and the $\lambda_{c}=0$ occurs at $R e \simeq 2000$, very close to the critical point for onset of sustained turbulence (Avila et al., 2011). In addition, the scaling of the critical velocity slip on the wall may give a hint to experimental relaminarisation of turbulence by accelerating the flow on the wall. 


\section{CHAPTER 6}

\section{SUMMARY}

The transition to fully turbulent flow and turbulence control are of fundamental importance in fluid dynamics and have been challenging scientists and engineers for over a century. This thesis is dedicated to them.

DNS is the tool for the studies presented in this thesis. Two DNS codes were adopted, one employs a pressure-free formulation (Willis \& Kerswell, 2009) and the other is a primitive-variable formulation (Hugues \& Randriamampianina, 1998). The latter was developed from scratch for cylindrical configuration and tests showed good performance of this new code. Both of them employ finite-difference method in the radial direction, which lowers the computation cost, and spectral methods (Fourier) in the periodic azimuthal and axial directions. In time-stepping, both are of the second-order of accuracy.

\subsection{Main conclusions}

1. The emergence of fully turbulent flow. The problem of transition to fully turbulent flow was lately understood through a collective work of theoretical modeling, DNS and experiments (Barkley et al., 2014). In this thesis, the DNS aspect of this work was discussed in detail and the transition scenario was explained.

Extensive DNS has been performed to accurately measure the speed of the upstream and downstream fronts of turbulence at a wide range of Reynolds number between 1900 and 5500, covering the regime of localized turbulence (puffs) and fully the turbulent regime. The speed was compared to that measured in experiments by my colleague Mukund Vasuvedan at IST Austria and excellent agreement was obtained (Fig. 3-12). The well-resolved speed data facilitated Barkley's model to capture 
the main features of pipe flow turbulence. The main findings are the following.

- Transition from puffs to slugs is explained as a transition from excitability to bistability. At low Reynolds numbers $(R e \lesssim 2200)$, pipe flow is excitable with puffs being localized excitations that feed on the surrounding laminar flow. Above $R e \simeq 2300$ pipe flow is bistable and turbulence can locally stabilize, which results in the growth of turbulence.

- However, the bifurcation point at which the system becomes bistable in the model, is masked by the turbulence nonlinear self-advection (see Fig. 3-13). Besides, fluctuations in real pipe flow, which are intrinsic to turbulence, lead to puff splitting and dig holes inside slugs, and tend to obscure puffs and slugs in the regime $2300 \lesssim R e \lesssim 2600$. Consequently, a clear critical point is absent and no simple scaling such as a square-root scaling or a scaling related to directed percolation exponents was observed. This nontrivial scaling has been a major obstacle for the clarification of the transition scenario in former studies (Lindgren, 1969; Wvgnanski \& Champagne, 1973; Durst \& Ünsal, 2006; Nishi et al., 2008). Sreenivasan \& Ramshankar (1986); de Lozar \& Hof (2010) claimed square root scaling of the turbulence growth rate but we argue that it was due to their under-resolved speed data. In this thesis the growth rate was shown to be rather linear during the emergence of fully turbulent flow (see Fig. 3-8).

- Our results (Barkley et al., 2014) clarified that the front speed selection mechanism for slugs is totally different from that for puffs. Both upstream and downstream fronts of slugs are trigger front whose speed is determined by the interplay between turbulence diffusion and production (see Sec. 3.2.2). These two speeds are usually different so that turbulence grows. Whereas the downstream front of puffs simply follows the upstream front at a fixed distance. For the definition of a trigger front see Tyson \& Keener (1988).

- There are two types of slug. One with a puff-like weak downstream front at moderate Reynolds numbers $2350 \lesssim R e \lesssim 2800$, and the other with strong front at high Reynolds number above about 3500. The former was referred to as puffs that grow or puffs out of equilibrium (Wygnanski et al., 1975; Duguet et al., $2010 b)$.

- The somewhat complicated scaling of the downstream front speed of slugs is caused by an adjustment from the weak to the strong front state that takes place in this $R e$ regime $(2300 \lesssim R e \lesssim 5000)$. The speed of the downstream front 
of slugs are regulated by the speed of weak and strong fronts in the asymptotic limit. The average speed of the downstream front is precisely captured by Barkley's model in a finite- $\epsilon$ case (corresponding to finite front width) (see Fig. 3-12). Fluctuations may even cause transient switch between weak and strong front at $R e \simeq 3000$ (see Fig. 3-14).

The transition scenario can be summarized as: at low Reynolds number the system is excitable and sufficiently strong disturbances create localized puffs. At higher Reynolds numbers the system becomes bistable and turbulence takes the form of slugs with a weak downstream front at moderate Re and eventually of slugs with a strong front at high Reynolds numbers (above $\simeq 3500$ ). There is no critical point because the turbulence self-advection and the intrinsic fluctuations mask the bifurcation point, and there is no sharp transition from weak to strong downstream front because of the finite front widths and strong fluctuations at low and moderate Reynolds numbers.

2. Turbulence control. Motivated by the fact that turbulence at high Reynolds numbers is highly dissipative and causes much higher friction loss than laminar flow, in the second part of this thesis, turbulence control strategies were developed to relaminarise turbulence. Following the studies in the transition to fully turbulent flow, a forcing strategy was devised to modify the velocity profile and reduce the transient growth of the flow, which was found to relaminarise turbulence in fully turbulent regime given sufficiently strong force. Statistical studies were performed for determining the minimum forcing amplitude and the resulting transient growth of the forced flow was also calculated. Besides, a vortex control was developed based on our observation that certain velocity fields, even with strong fluctuations, may decay at high Re (discussed in Appendix $\mathrm{A}$ ). The vortices were designed such that they alter the flow structures and flatten the velocity profile, resulting in an unnatural flow field that relaminarises after the control is deactivated. Results are summarized as follows.

- A force that decelerates the flow near the pipe center and accelerates the flow near the pipe wall, deforming the velocity profile into a plug-like one, can relaminarise turbulence if the force is strong enough. This corresponds to changes in the nullclines of the model system, which remove the fixed point on the upper branch $q^{+}$and render the originally bistable system excitable, or even refractory (see Fig. 4-1). Indeed, inverse transition from fully turbulent flow to puffs was observed under such kind of forcing (see Fig. 4-7).

- Sufficiently strong force always relaminarises turbulence. Under marginal forc- 
ing, turbulence behaves stochastic (see Fig. 4-6).

- A transient growth analysis showed that this forcing strategy greatly reduces the transient growth, which is an important part for the turbulence self-sustaining cycle (Schoppa \& Hussain, 2002). Here results suggested that sufficiently low transient growth can not sustain this cycle and leads to relaminarisation.

- The minimum transient growth that sustains turbulence was investigated and was found to be constant, about 15, at all Re we considered (see Fig. 4-9). This transient growth was also found to be the minimum one for puffs to survive in unforced flow at low Re (Fig. 4-10). We argue that this constant transient growth sets a boundary between excitable and refractory, below which the system is refractory so that no disturbances can survive.

- Streamwise vortices, when imposed on top of a turbulent flow, can flatten the velocity profile and alter the number and size of streaks. Our study implies that if the number and size of streaks of the original flow are sufficiently changed, flow may relaminarise (see Appendix A). This was indeed achieved at $R e=3000$ with 12 pairs (azimuthal wavenumber 12) of counter-rotating vortices. This is consistent with the transient growth argument above in the sense that on the one hand, streamwise vortices flatten velocity profile so that reduce the transient growth. On the other hand, near wall structures are altered such that they are far from the optimal perturbations. Both result in lower energy amplification via transient growth and may lead to relaminarisation.

3. The partial-slip boundary condition. The effects of the partial-slip boundary condition on turbulence were studied. Results showed that azimuthal slip intensifies turbulence, whereas streamwise slip weakens turbulence and can even relaminarise turbulence if the slip is large enough (see Fig. 5-1 and Fig. 5-3). The minimum slip length that leads to relaminarisation was studied up to $R e=20000$. Results showed that a slip length comparable to the radius is needed to relaminarise turbulence and it increases linearly as $R e$ increases, see Fig. 5-4. The corresponding velocity slip on the wall may suggest experimental control that relaminarises turbulence by generating similar velocity at the pipe wall.

\subsection{Future work}

A couple of problems require future work to be clarified. 
- The front speed of slugs at high Re form a parabola (see Fig.3-12). Clearly this can not hold forever as Reynolds numbers increases further since the two branches of this parabola will keep increasing and decreasing, which means the front speed will approach infinity as $R e \rightarrow \infty$. This clearly can not happen. The front speed at higher Reynolds numbers (above 6000) needs to be studied, which requires very expensive simulation and more efficient parallel codes. The reason for the deviation from the asymptotic analysis (which gives the parabola) should also be investigated.

- How fluctuations cause puff splitting is still unknown. Presumably, vortices shed off from the seeding puff at the downstream front and occasionally reach far enough (beyond the puff-puff interaction distance) to generate a new puff. However, quantitative studies are still lacking.

- The speed of turbulence self-advection at higher Reynolds number (above $R e=$ 10000) is worth investigating. The result in Appendix B showed that this advection speed is lower than the mean flow speed above $R e=10000$ and it still keeps decreasing. The implication of this result needs to be interpreted. Besides, it is a factor in Barkley's model and may be related to the discrepancy between the model and real flow when Re gets large.

- Vortex control was shown to be able to relaminarise turbulence at $R e=3000$ and are possible to be implemented via wall injection-suction It should be a promising control technique for practical applications, however, it fails at higher Reynolds numbers. Control with vortices at higher Reynolds numbers is worth studying.

- In Chapter 5 it was shown that the minimum slip length that suffices to relaminarise turbulence scales linearly with Re. This scaling should be better interpreted. Besides, this work should be related to the transient growth argument proposed in the forcing study, i.e., the minimum slip length may be related to the minimum transient growth for turbulence to be sustained. The transient growth analysis in case of partial slip boundary condition needs to be performed. 
Summary 


\section{APPENDix A}

\section{REYNOLDS NUMBER REDUCTION}

Studies in Chapter 4 showed that turbulence decays in case of a flatter velocity profile under forcing. One implication of this finding on relaminarisation is that a turbulent flow at very high Reynolds number which consequently has a very flat velocity profile may relaminarise after a reduction to a lower Reynolds number (still in the turbulent regime) where turbulence naturally would have less flat profile. If this is true, this may suggest control methods that perturb turbulence impulsively such that the flow field has unnatural velocity profile and structures (as those from higher level of turbulence at higher Re), which will decay after the control is removed. In what follows, we first study the different velocity profiles and structures of turbulence at different $R e$, then implement the Re reduction and show that flow field from higher Re indeed relaminarises after the reduction, though it contains very strong disturbances.

\section{A.1 Difference between different levels of turbu- lence}

It is known that in wall-bounded turbulence, structures with different length scales, located at different wall distances, organize themselves so that the momentum transfer in the wall-normal direction stays in balance, resulting in a certain 'natural' velocity profile. A fixed 'unnatural' velocity profile breaks this balance and causes substantial changes in turbulence intensity (Tuerke \& Jimenez, 2013). This implies that there may exist a way of driving turbulence far from or even out of its attraction basin by disturbing it in such a way that the flow field is somehow 'unnatural' to a turbulence. We already showed the possibility of this in the previous section. Considering that velocity profile differs at different turbulence intensities, an alternative way to obtain 
a plug-like (flatter) velocity profile, which does not require an additional forcing, is to expose the flow to a higher level of turbulence. since nondimensionally speaking, at higher level turbulence the velocity profile is flatter because of the stronger radial momentum transfer. To achieve this situation we initiate a turbulent flow at a high Reynolds number, such as $R e=10000$. Then, we abruptly reduce the $R e$ to a much lower one, to $R e=3000$, at which the flow field from $R e=10000$ is supposed to be unnatural to the turbulence in the sense that the velocity profile is flatter and the size and wall-distances of the turbulent structures are not expected at this Re. By doing so, we can study how this flow field adjusts to the new Reynolds number and if it can jump out of the attraction basin of the turbulence at $R e=3000$.

Fig. A-1 gives more details of the differences between a flow field at $R e=3000$ and one at $R e=10000$. Fig. A-1(a) compares the mean velocity profiles at these two Reynolds numbers, showing that the profile at $R e=10000$ is more plug-like than that at 3000, due to the stronger radial momentum transfer at higher Reynolds number. Fig. A-1(b) depicts the flow details in terms of the size and number of the near wall streaks by showing the axial velocity relative to the mean velocity at $y^{+}=30$ from the wall, where the center of streaks (Kim et al., 1987). Usually, the quantities with superscript ${ }^{+}$are ones normalized by kinematic viscosity $\nu$ and friction velocity $u_{\tau}$. Here the distance $y^{+}$is normalized by $\nu / u_{\tau}$. The data are taken arbitrarily at axial position $z=0$ and however considered as typical at their respective Reynolds number. The streaks are defined as the areas in which the velocity is lower or higher than the mean velocity of their surroundings, corresponding to negative areas (low speed streaks) and positive areas (high speed streaks) in Fig. A-1(b). Roughly speaking, we can see that at $R e=3000$ there exist about 4 high speed and 4 low speed streaks at the chosen axial position, however about 10 of each at $R e=10000$. The in-plane velocity fields and the contours of the deviation of the streamwise velocity $u_{z}$ from the mean velocity profile are shown in Fig. A-1(c)(d). The difference between the size and number of streaks and vortices is vividly shown. Another point to note is that the streamwise vortices convect mean shear, distorting the mean velocity profile such that a maximum of curvature appears near the wall rather than at the pipe center as in laminar flow. The location of the maximum of the curvature is found approximately to coincide with the position of streaks that are generated by streamwise vortices. As a matter of fact, $y^{+}=30$ gives a position $r=0.719$ at $R e=3000$, and 0.908 at 10000 , which roughly coincide with the position of the the maximum of the curvature, or the 'corner' of the mean profiles, as can be seen in Fig. A-1(a). To conclude, the higher azimuthal wave number and being closer to the wall make the streaks smaller in size 
(a)

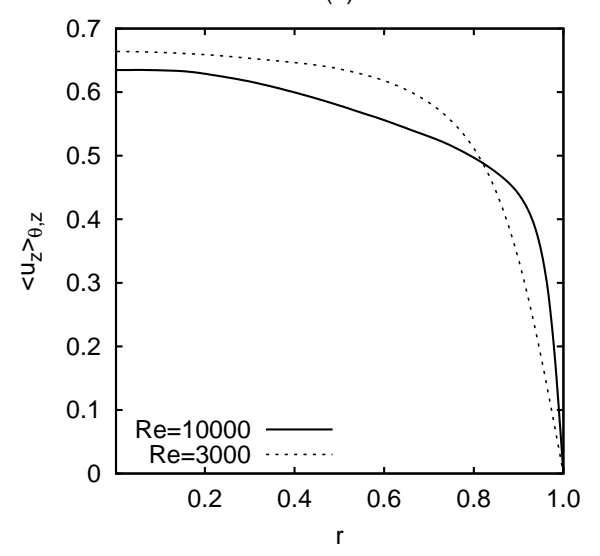

(c)

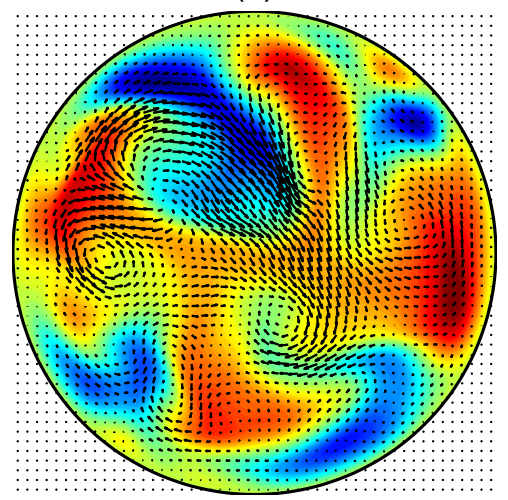

(b)

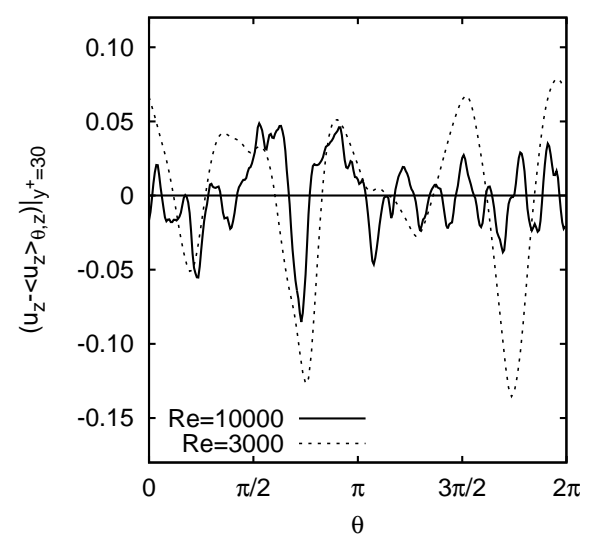

(d)

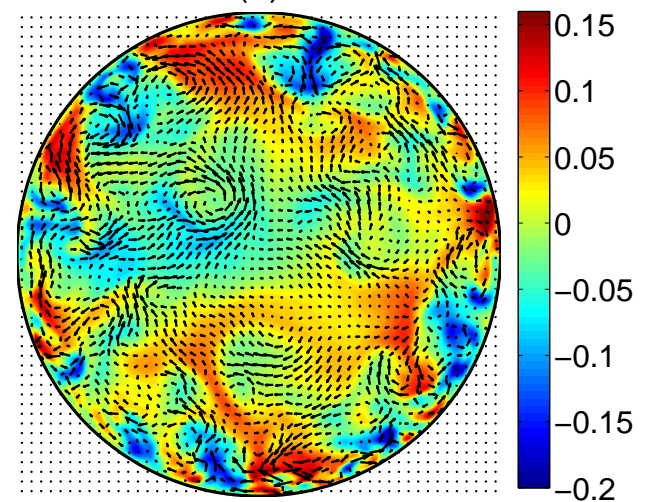

Figure A-1: (a) Typical mean velocity profiles at $R e=3000$ and 10000 . (b) The depiction of the size and number of streaks at $\mathrm{Re}=3000$ and 10000. The deviation of axial velocity from the mean axial velocity profile at a distance of $y^{+}=30$ from the wall, plotted against azimuthal position. Note that all velocities are normalized by the velocity $U_{\max }$ at their respective $R e$. The in-plane velocity field together with the contour of axial velocity $u_{z}$ relative to the mean velocity profile are plotted in (c) $(R e=3000)$ and $(\mathrm{d})(R e=10000)$. They are taken on the same cross sections as (a) and (b). 
than they are at lower Reynolds number.

\section{A.2 Implementation of the Re reduction}

For the purpose of generating initial conditions, there are at least two ways to reduce the Re. First, we simply take a nondimensional velocity field of a flow at a higher $R e$ and rerun it at the desired $R e$, which is not a physical reduction because the normalization of the velocity field is changed rudely. This one can be referred to as the unphysical reduction. The other way to implement the reduction is to keep the dimensional velocity field. In this case, the nondimensional velocities have to be scaled appropriately o keep the reduction physical. This will be a good approximation of reducing the flow rate abruptly in the experiment while keeping the magnitudes of the fluctuations on top of the mean flow. However, it needs an infinite force pulse to change the mass flux (also the velocity profile) at the moment of reduction. Both of these two ways serve well for the purpose of generating unnatural flow field with undesired velocity profiles and structures, so they are all eligible for the current numerical study. The former will be referred to as unphysical reduction and the latter physical reduction.

\section{A.2.1 Unphysical reduction}

Once $R e$ is reduced to 3000 , a sharp decrease in the turbulence kinetic energy must occur. Presumably, smaller structures and flatter mean velocity profile from $R e=10000$ will lead to a higher level of dissipation, causing an excessive destruction of kinetic energy at $R e=3000$. Fig. A-2 shows the history of the kinetic energy of turbulent fluctuations of the reduction from $R e=10000$ to 3000. The initial transients show a sharp decrease in the kinetic energy. 10 runs with different initial conditions were carried out among which 7 relaminarised. In the other 3 runs turbulence managed to recover from the initial decaying and reach a 'natural' intensity at $R e=3000$. As mentioned before, we attribute the initial decaying and the final relaminarisation to the 'unnatural' flow structures in the initial conditions, which are much more dissipative than the turbulence expects after the reduction. It is worth mentioning that the difference from the forcing experiment is that here if turbulence survives, it recovers to the level of the natural turbulence at the this Reynolds number, whereas to a lower level in the forcing study. This is because here the velocity profile is free to evolve after the reduction, but was forced all the time in the earlier forcing study. 


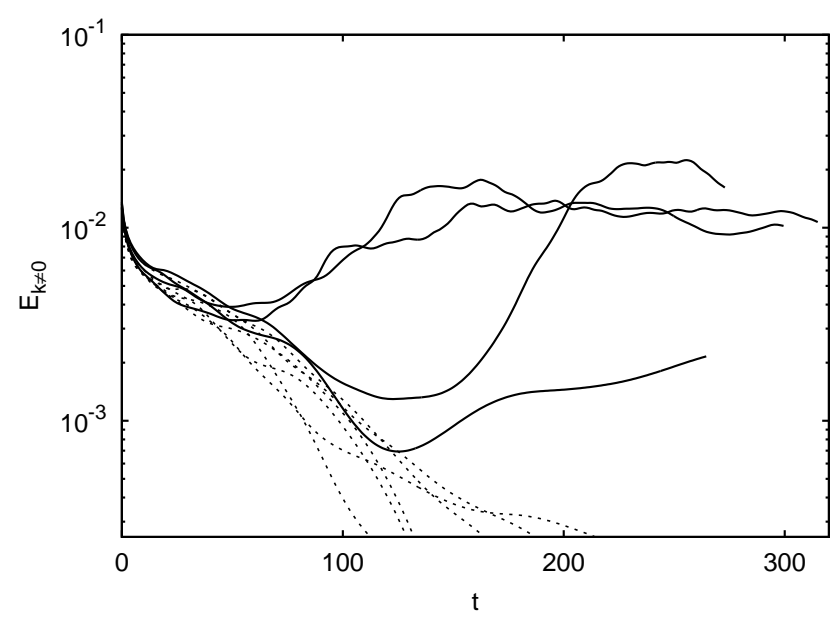

Figure A-2: The Re reduction experiment from $R e=10000$ to 3000 . Here the time series of the kinetic energy of the x-dependent fluctuations $E_{k \neq 0}$ are plotted, which is normalized by the kinetic energy of the Hagen-Poisseuille flow. 10 realisations are shown here with statistically independent initials conditions drawn from a long simulation of fully turbulent flow at $R e=10000$. 7 relaminarisations out of 10 runs were observed (dashed lines).

As shown above, the abnormally sized and located structures combined with flatter mean velocity profiles in initial conditions lead to relaminarisation, which implies that the bigger the difference from the normal turbulence, the more likely for the turbulence to relaminarise. Following this thought, the likelihood of relaminarisation as a function of Reynolds number before the reduction was studied. Considering the stochastic behavior of the flow after the reduction, as shown in Fig. A-2, statistics were carried out for reductions from a variety Reynolds numbers. Initial conditions were prepared at Reynolds numbers $R e=15000,12000,10000,7500,6500$, and 5000. At each $R e$, tens of sufficiently separated snapshots were produced. Subsequently, the $R e$ was to 3000 and the probability of the relaminarisation was estimated as the fraction of runs that ended with turbulence. For each reduction, $30 \sim 50$ realizations with uncorrelated initial conditions were performed for the statistics. Results are shown in Fig. A-3, which gives a clear trend that the lager the reduction in $R e$, the more likely the relaminarisation is to occur. Note that for the reduction from $R e=15000$ to $R e=3000$, almost in all runs turbulence completely decayed. This indicates that if the velocity field at $R e=3000$ can be perturbed appropriately, generating flatter mean velocity profile and finer structures (streaks and vortices), turbulence can be efficiently eliminated. This study suggests experimental control technique which aims at flattening the velocity profile and breaking the existing structures into finer ones. 


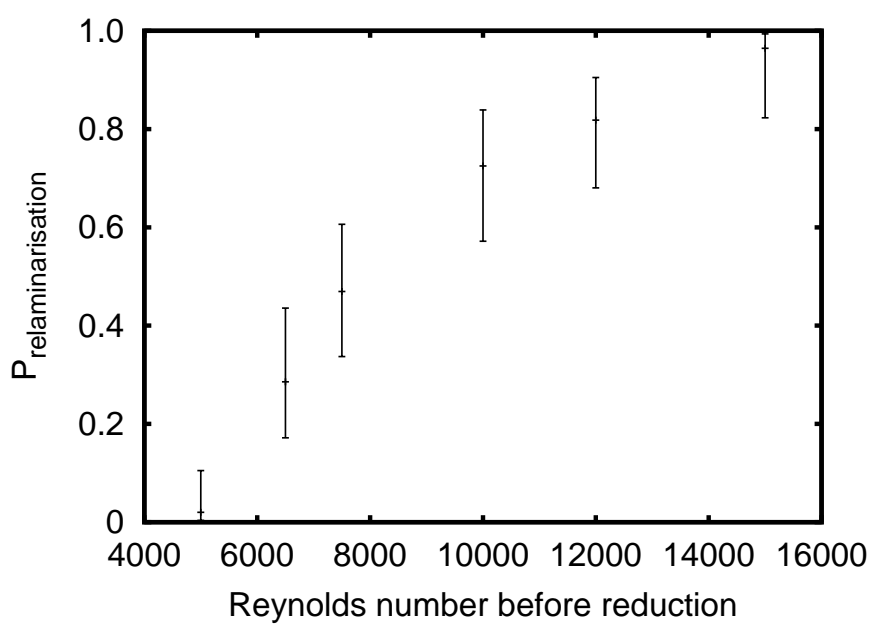

Figure A-3: Statistical study of the Re reduction experiment from various Reynolds numbers to $3000.30 \sim 50$ realizations for each point. The errorbars represent the $95 \%$ confidence interval associated with the estimation of the probability of relaminarisation by dividing the total sample size by the number of relaminarisation.

\section{A.2.2 Physical reduction}

In unphysical reduction, nothing was done to the initial condition drawn from higher Reynolds numbers. To be more realistic, physical reduction should be adopted. In experiment, if the flow rate can be abruptly changed, the mean velocity profile must also change immediately since the conservation of mass and the incompressibility. However, the lack of direct control on turbulent fluctuations will leave the fluctuations almost unchanged at the right moment of the flow rate reduction. So, a more realistic way to conduct the reduction in numerical simulation will be rescaling the flow flied appropriately before running the simulation at a different Re. In this thesis, the velocity is normalized by $U_{\max }=2 U$, so the ratio between the velocities are the same as that between $R e$ if the pipe radius $R$ and flow viscosity $\nu$ are kept constant. Therefore, what should be done is to rescale the fluctuations (with respect to the mean velocity profile) by a factor of $\frac{R e_{i}}{R e}$ where the $R e_{i}$ is the Reynolds number before reduction. After the rescaling, fluctuations are very strong in amplitude, yet the size of the structures such as vortices and streaks are still small as shown in Fig. A-1.

The same statistics as for the other reduction were carried out at the same Reynolds numbers. The results are shown in Fig. A-4. Again, relaminarisation occurs, but with a lower rate compared to the unphysical reduction, which is expected as in current reduction the initial disturbances, though are of the same structures, are much stronger than that in the unphysical reduction case. Nevertheless, it is sur- 
prising that at $R e=3000$ such strong perturbations fail to create turbulence instead decays.

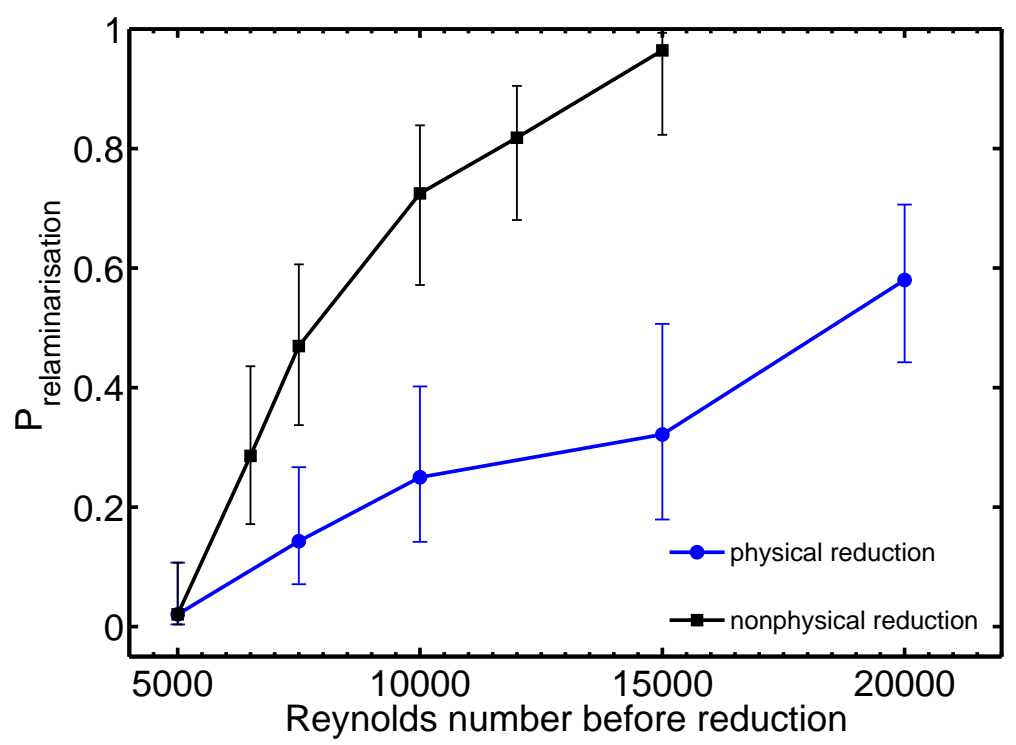

Figure A-4: Statistical study of the physical Re reduction experiment (blue) from a variety of Reynolds numbers to 3000. For comparison, the result of the non-physical reduction is also shown (black). $30 \sim 50$ realizations for each point. The errorbars represent the $95 \%$ confidence interval associated with the estimation of the probability of relaminarisation by dividing the total sample size by the number of relaminarisation.

\section{A.3 Discussion}

Turbulence arrange the sizes and positions of the near wall coherent structures according to the specific Reynolds number, balancing the momentum transport in the radial direction and creating a 'natural' flow field of certain mean velocity profile (in a time-averaged sense) (Tuerke \& Jimenez, 2013). A Reynolds number reduction study shows that an 'unnatural' flow field which has a flatter mean velocity profile and finer near wall coherent structures leads to relaminarisation, implying possible flow control mechanisms that aim at rendering the flow field unnatural. 
Appendix A. Reynolds Number Reduction 


\section{APPENDix B}

\section{AdveCTION SPEED OF TURBUlENCE}

Because of the general symmetries of the Navier-Stokes equations and the pipe geometry, pipe flow bears continuous translational and rotational symmetries. A pure translation and rotation about the pipe axis will not change the intrinsic dynamics of the flow. For example, the laminar pipe flow advects downstream at a constant speed, and the profile is independent of the specific location along the pipe axis and rotationally symmetric. This fact allows the freedom of choice of frame of reference. A traveling wave solution in the laboratory frame can be turned into an equilibrium state by choosing a frame co-moving with the structure and the solution can be better investigated in this frame. In more complex turbulent flow, the turbulent dynamics are usually entangled with these symmetries. Ideally, if the trivial degree of freedom due to symmetries can be removed in an appropriately chosen frame of reference, the intrinsic dynamics of the flow can be revealed. The symmetry reduction has been discussed for invariant traveling wave and relative periodic solutions (Willis et al., 2013; Mellibovsky \& Eckhardt, 2012)

However, for complex turbulent flow, it is not straightforward to define a global advection speed (also called convection speed or propagation speed) of turbulence since eddies of different sizes may advect at different speeds. Del Álamo \& Jiménez (2009) developed methods to determine the advection speed of individual modes (with individual wave lengths). Besides, in wall shear turbulence, the advection speeds of turbulent fluctuations are likely to be dependent of the distance to the wall. Pei et al. (2012) studied the advection speed profile of the streamwise velocity fluctuation based on the space-time correlation of the streamwise velocity fluctuation in channel flows. These authors showed that this speed stays approximately constant for $y^{+}<10$ and much higher than the local mean flow speed, and increases above the viscous sublayer. Nevertheless, it would be convenient if an overall advection speed on large scale of 
turbulence can be calculated.

Recently, Kreilos \& Eckhardt (2013) proposed a method to remove the translational symmetry and evaluate the overall advection speed of turbulence in parallel shear flows. The procedure is summarized as following:

1. Rewrite the Navier-Stokes equations as

$$
\partial_{t} \boldsymbol{u}(r, \theta, z, t)=\boldsymbol{f}(\boldsymbol{u}(r, \theta, z, t), t) .
$$

2. Approximate the flow $\boldsymbol{u}(r, \theta, z+\Delta z, t+\Delta t)$ with Taylor-expansion

$$
\boldsymbol{u}(r, \theta, z+\Delta z, t+\Delta t) \approx \boldsymbol{u}(r, \theta, z, t)+\Delta z \partial_{z} \boldsymbol{u}(r, \theta, z, t)+\boldsymbol{f}(\boldsymbol{u}(r, \theta, z, t), t) \Delta t
$$

3. Assume the turbulence is advected at a speed of $c$, then $\Delta z=c \Delta t$, then we have

$$
\boldsymbol{u}(r, \theta, z+\Delta z, t+\Delta t) \approx \boldsymbol{u}(r, \theta, z, t)+\left(c \partial_{z} \boldsymbol{u}(r, \theta, z, t)+\boldsymbol{f}(\boldsymbol{u}(r, \theta, z, t), t)\right) \Delta t
$$

4. Projecting with $\partial_{z} \boldsymbol{u}(r, \theta, z, t)$, the advection speed $c$ can be obtained as

$$
c=-\frac{<\partial_{z} \boldsymbol{u}(r, \theta, z, t) \cdot \boldsymbol{f}(\boldsymbol{u}(r, \theta, z, t), t)>}{\left\|\partial_{z} \boldsymbol{u}(r, \theta, z, t)\right\|^{2}},
$$

where

$$
<\boldsymbol{u} \cdot \boldsymbol{f}>=\int_{V}(\boldsymbol{u} \cdot \boldsymbol{f}) d V
$$

and $\|\boldsymbol{u}\|^{2}=\langle\boldsymbol{u} \cdot \boldsymbol{u}\rangle$. Note that $\boldsymbol{u}(r, \theta, z+\Delta z, t+\Delta t)$ and $\boldsymbol{u}(r, \theta, z, t)$ are required to equal according to the definition of advection, however, in the weak form

$$
<\partial_{z} \boldsymbol{u}(r, \theta, z, t) \cdot(\boldsymbol{u}(r, \theta, z+\Delta z, t+\Delta t)-\boldsymbol{u}(r, \theta, z, t))>=0 .
$$

This procedure allows to evaluate the instant advection speed of turbulence, which itself is likely to be time-dependent. In this chapter, this method will be used to approximate the speed of self-advection of pipe flow turbulence, which is an important factor in Barkley's one-dimensional pipe flow model as discussed in Sec. 3.2. DNS Code 2 is employed for this study. 


\section{B.1 Advection speed vs. Re}

The advection speed is calculated at several Reynolds numbers (in slug regime) in the range $R e \in(2600,10000)$. The time series of the advection speed at several Reynolds numbers are shown in Fig. B-1. The results show that at $R e \lesssim 7000$ the advection

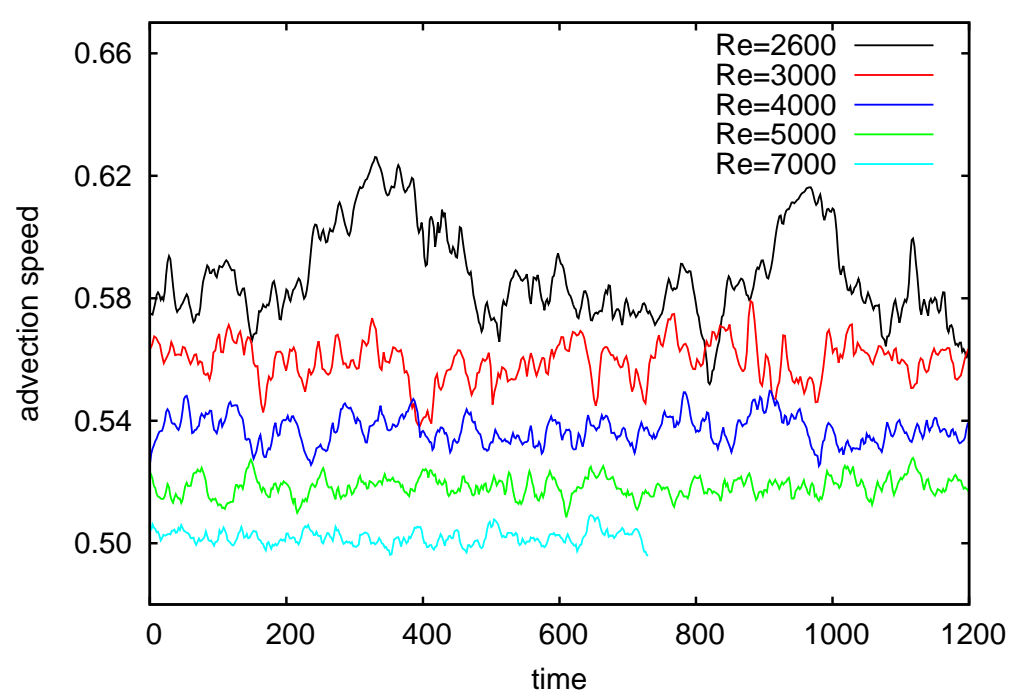

Figure B-1: Time series of the advection speed at several Reynolds numbers.

speed is higher than the mean flow speed, and as Re increases the advection speed of turbulence decreases. The other apparent trend is that as Re increases the advection speed undergoes smaller fluctuations. At low Reynolds numbers such as $R e=2600$, the speed shows huge fluctuations, however, at $R e=7000$ the advection speed is very stable. This is consistent with the fact that at low Reynolds numbers turbulence manifests large spatio-temporal intermittency while at high Re intermittency becomes very rare (Avila \& Hof, 2013).

To have the advection as a function of $R e$, the mean advection speed is estimated from these time series and the fluctuations are measured by the standard deviation. The speed is measured up to $R e=10000$ and the results are shown in Fig. B-2, As we can see, the advection speed decreases as $R e$ increases. Interestingly, at $R e=10000$, the advection speed of turbulence is even lower than the mean flow speed, i.e., the turbulence advects slower than the mean flow. However, the implication of this fact is still unclear. From the trend one can infer that the advection speed of turbulence will decrease as $R e$ increases further. To clarify if the speed eventually saturates at certain $R e$ or keeps decreasing forever, future investigations are needed. 


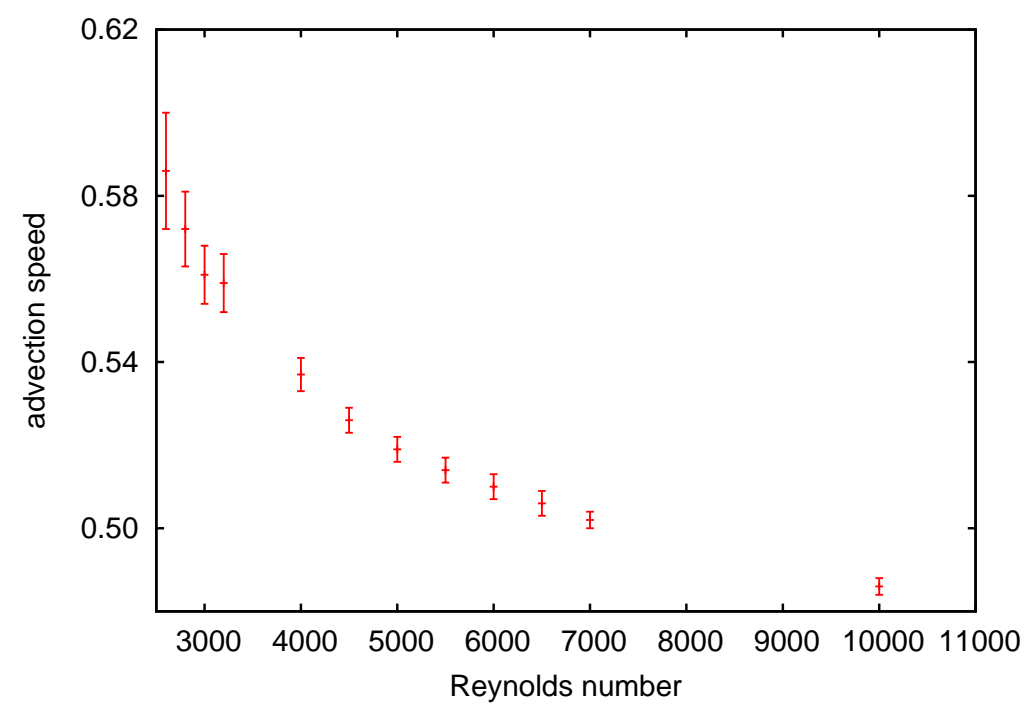

Figure B-2: The advection speed as a function of $R e$. The speed are measured at $R e=2600,2800,3000,3200,4000,5000,5500,6000,6500,7000$, and 10000. The errorbars are standard deviations.

\section{B.2 Advection speed vs. the centerline velocity}

(a)

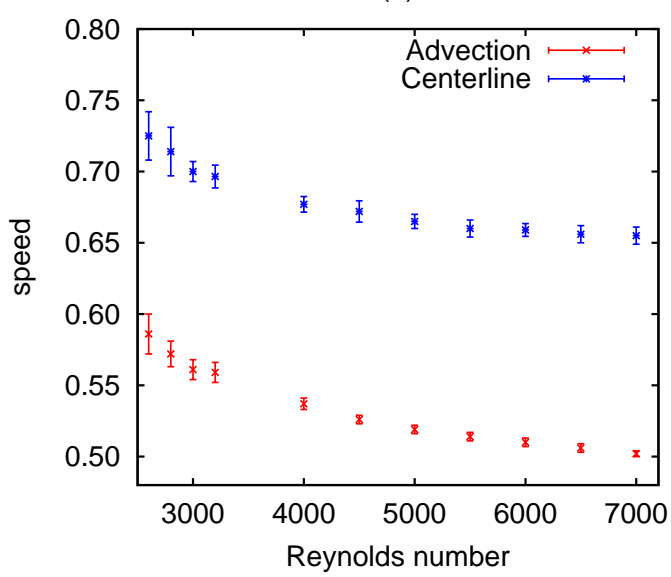

(b)

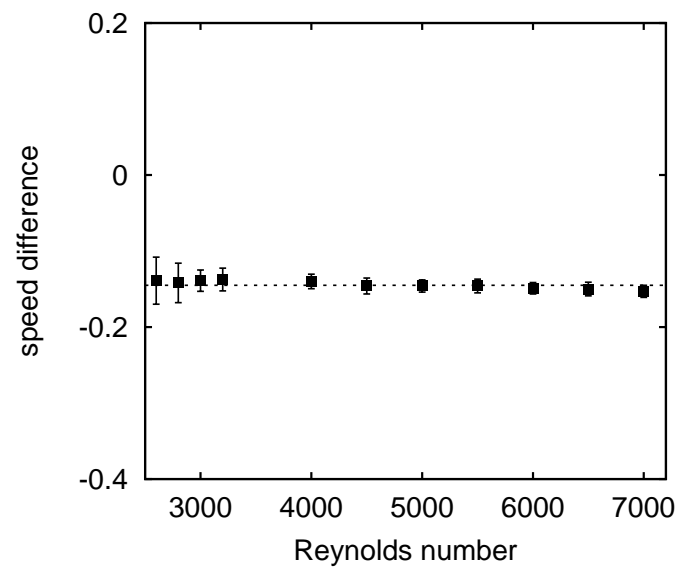

Figure B-3: (a) The comparison between the advection speed and the centerline velocity. (b) The difference between the advection speed and the centerline velocity.

In Barkley's pipe flow model as shown in Sec. 3.2, it is assumed that the turbulence is advected nonlinearly at a speed lower than the centerline velocity, depicted by the $u-\zeta$ in the model equations, where $u$ represents the centerline velocity and $\zeta$ is the amount by which the advection speed is lower than the centerline velocity and is a positive number. In this section, the relation between the advection speed of turbulence and the centerline velocity in real pipe flow is investigated. 
The centerline velocity of the mean velocity profile is measured in the Reynolds range $R e \in(2800,7000)$, in which the model correctly predicts the pipe flow as discussed in Chapter 3. The comparison between the advection speed and the centerline velocity are presented in Fig. B-3(a). As shown, turbulence indeed propagates at a lower speed than the centerline velocity at any Reynolds number investigated here. Fig. B-3(b) shows the difference between these two speeds. Surprisingly, in this Reynolds number range, the difference stays approximately constant, about 0.145, just as assumed in Barkley's model. These results qualitatively justify the assumptions in the model.

\section{B.3 Advection speed vs. the front speed}

The advection speed formulated above applies to fully turbulent flow but not to intermittent flow with fronts (turbulent-laminar interfaces) because the speed of fronts is not just a result of advection but instead is determined by the interplay of autocatalytic production and diffusion of turbulence at the fronts (Tyson \& Keener, 1988), for the details for pipe flow please see Sec. 3.2. Nevertheless, the advection speed with the formulation Eqs. (B.4) still applies to the core region of the turbulence with fronts. For examples, it applies inside of slugs sufficiently far from the fronts. In order to view the fronts in the frame of reference co-moving with turbulence, the comparison between the front speed and the advection speed is made and shown in Fig. B-4.

It is clearly shown that the advection speed (black squares) decreases as $R e$ increases and crosses the speed of the downstream front at about $R e \simeq 2900$. Below this $R e$, the advection speed of turbulence in the core region of slugs is higher than both upstream (cyan triangles and blue circles) and downstream (green triangles and red circles) fronts, whereas sits between the speed of upstream and downstream fronts above $R e \simeq 2900$.

Now we look at turbulence in the frame of reference comoving with the advection speed. As shown in Fig. B-5. Note that the turbulence self-advection is a nonlinear and time-dependent (see Fig. B-1). Here the advection speed means the mean advection speed. In such a frame of reference, the structure in the bulk of slugs overall stay still, while the fronts move. Below $R e \simeq 2900$, both the upstream and downstream front move to the left and the upstream front moves faster (see Fig. B-5(a)). Hence, overall turbulence production occurs at the upstream front, or, the produc- 


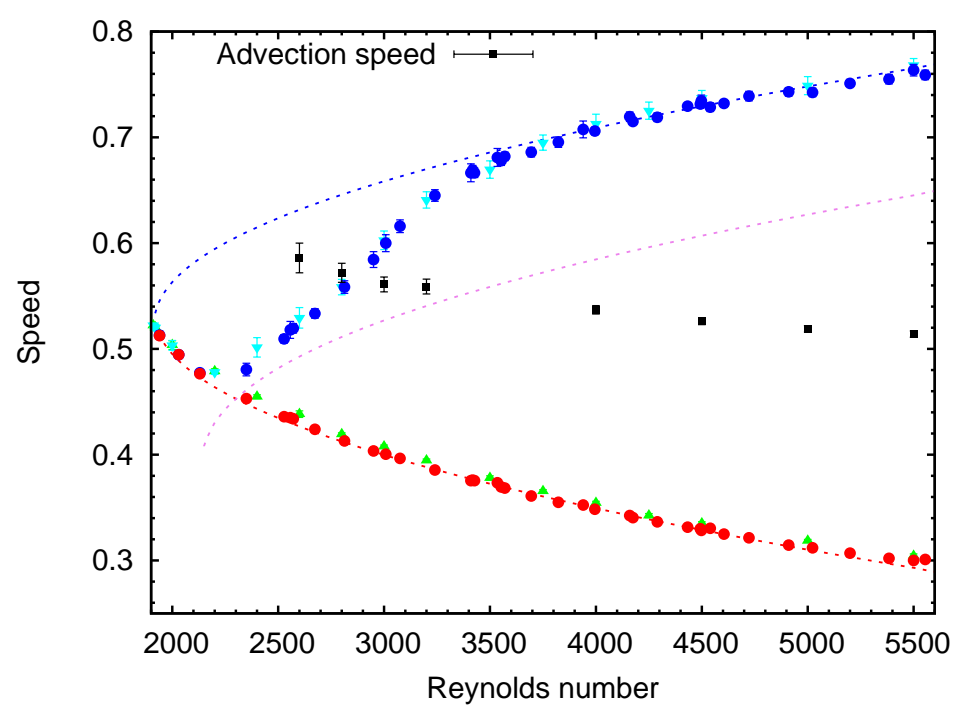

Figure B-4: The comparison between the advection speed (black squares) and the front speed from DNS (triangles), experiment (circles) and asymptotics (dashed lines).

tion outweighs the diffusion of turbulence. On the contrary, the downstream front moves into turbulence, implying that overall relaminarisation occurs at the downstream front, in other words, the diffusion dominates there. Above $R e \simeq 2900$, the upstream front moves to the left and the downstream front moves to the right (see Fig. B-5(b)) and turbulence is being produced at both fronts, which means turbulence production dominates at both fronts. Roughly speaking, slugs take a weak front below $R e \simeq 2900$ and a strong front above, see Fig. B-4. However, the fluctuations may make this division blurry and may be responsible for a smooth change from a weak front to a strong front. To summarize, these facts suggest that below $R e \simeq 2900$, the diffusion outweighs turbulence production at the downstream front and slugs have weak fronts, and above $R e \simeq 2900$, turbulence production outweighs diffusion and slugs have strong fronts. The competition between the autocatalytic production and diffusion of turbulence not only determines the speed of the fronts (Tyson \& Keener, 1988), but also selects the shape of the fronts.

\section{B.4 Discussion}

In this chapter the nonlinear self-advection of turbulence is studied, which is an intrinsic factor for pipe flow turbulence and was found to be responsible for the absence of a clear critical point in the transition from puffs to slugs, as discussed in Chapter 3, Based on the method proposed by Kreilos \& Eckhardt (2013), to my 


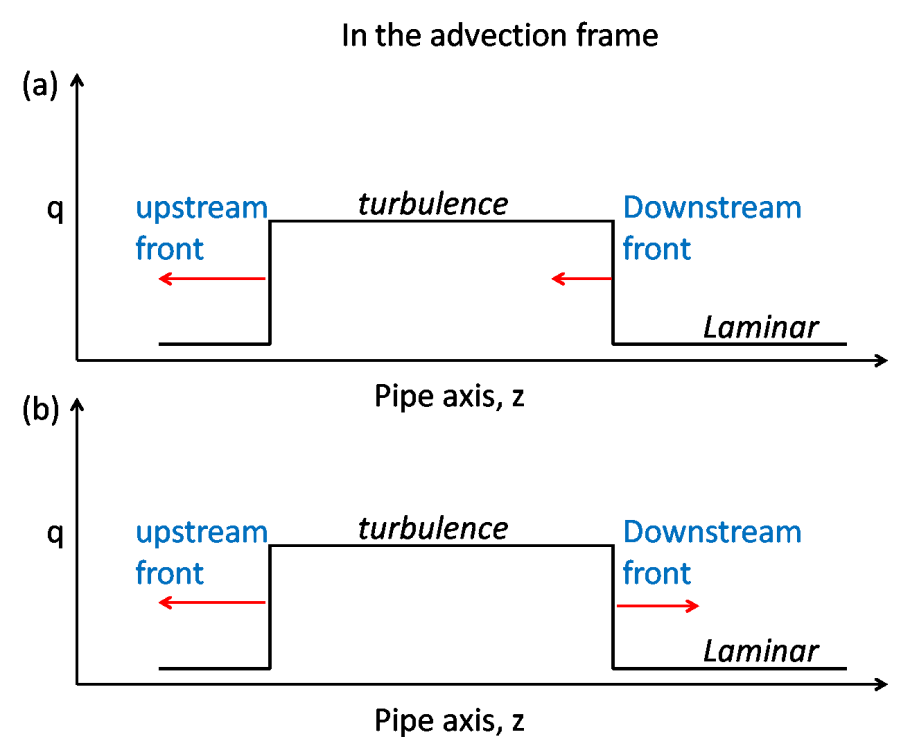

Figure B-5: Slugs looked at the advection frame of reference, i.e., the frame comoving with turbulence itself. (a) Slugs below $R e \simeq 2900$. (b) Slugs above $R e \simeq 2900$.

knowledge, the advection speed of pipe flow turbulence is for the first time computed. Compared to the average centerline velocity of turbulence, the advection speed is lower by approximately a constant at a wide range of Reynolds number, which justifies the assumption in Barkley's model that the self-advection is slower than the centerline velocity by a constant. Further, the advection speed is compared to the front speed and the fronts are viewed in the frame of reference of turbulence advection. It is speculated that below $R e \simeq 2900$ the diffusion outweighs turbulence production at the downstream front, resulting in a weak front. Above $R e \simeq 2900$, turbulence production dominates and slugs take a strong front. This is roughly agrees with the former studies (Wygnanski \& Champagne, 1973; Duguet et al., 2010b) where slugs with diffusion-dominant weak front below $R e \simeq 2800$ were referred to as puffs out of equilibrium. 
Appendix B. Advection Speed of Turbulence 


\section{BIBLIOGRAPHY}

Antonia, R. A., Fulachier, L., Krishnamoorthy, L. V., Benabid, T. \& Anselmet, F. 1988 Influence of wall suction on the organized motion in a turbulent boundary layer. J. Fluid Mech. 190, 217-240.

Avila, K., Moxey, D., De Lozar, A., Avila, M., Barkley, D. \& Hof, B. 2011 The onset of turbulence in pipe flow. Science 333, 192-196.

AvilA, M. \& Hof, B. 2013 Nature of laminar-turbulence intermittency in shear flows. Phys. Rev. E 87, 063012.

Avila, M., Willis, A. P. \& Hof, B. 2010 On the transient nature of localized pipe flow turbulence. J. Fluid Mech. 646, 127-136.

BANDYOPADHYAY, P. R. 1986 Aspects of the equilibrium puff in transitional pipe flow. J. Fluid Mech. 163, 439-458.

BARkley, D. 2011a Modeling the transition to turbulence in shear flows. J. Phys.: Conf. Ser. 318, 032001.

Barkley, D. $2011 b$ Simplifying the complexity of pipe flow. Phys. Rev. E 84, 016309.

Barkley, D., Song, B., Mukund, V., Avila, M. \& Hof, B. 2014 Emergence of fully turbulent flow. Submitted.

Barkley, D. \& Tuckerman, L. S. 2005 Computational study of turbulent laminar patterns in couette flow. Phys. Rev. Lett. 94, 014502.

Batiste, O., Knobloch, E., Alonso, A. \& Mercader, I. 2006 Spatially localized binary-fluid convection. J. Fluid Mech. 560, 149-158.

Bazant, Martin Z. \& Vinogradova, Olga I. 2008 Tensorial hydrodynamic slip. J. Fluid Mech. 613, 125-134.

Bechert, D. W., Bruse, M., Hage, W., Van Der Hoven, J. G. T. \& Hoppe, G. 1997 Experiments on drag-reducing surfaces and their optimization with an adjustable geometry. J. Fluid Mech. 338, 59-87.

Belson, B. A., Semeraro, O., Rowley, C. W. \& Henningson, D. S. 2013 Feedback control of instabilities in the two-dimensional blasius boundary layer: The role of sensors and actuators. Phys. Fluids 25, 054106. 
Berhard, P. S., Thomas, J. M. \& Handler, R. A. 1993 Vortex dynamics and the production of reynolds stress. J. Fluid Mech. 253, 385-419.

Bewley, T. R. 2001 Flow control: new challenges for a new renaissance. Applied Mechanics Reviews 37, 21-58.

Bewley, T. R., Moin, P. \& Temam, R. 2001 Dns-based predictive control of turbulence: an optimal benchmark for feedback algorithms. J. Fluid Mech. 447, $179-225$.

Bottin, S. \& Chaté, H. 1998 Statistical analysis of the transition to turbulence in plane couette flow. Eur. Phys. J. B 6, 143-55.

Bottin, S., Daviaud, F., Manneville, P. \& Dauchot, O. 1998 Discontinuous transition to spatiotemporal intermittency in plane couette flow. Europhys. Lett. 43(2), 171-76.

BRAndT, L. 2014 The lift-up effect: the linear mechanism behind transition and turbulence in shear flows. arXiv pp. arXiv:1403.4657v1 [physics.flu-dyn].

Brooke, J. W. \& Hanratty, T. J. 1993 Origin of turbulence-producing eddies in a channel flow. Phys. Fluids A 5, 1011-1022.

BrosA, U. 1989 Turbulence without strange attractor. J.Śtat.Pंhys. 55, 1303-1312.

Cherry, E. M. \& Fenton, F. H. 2008 Visualization of spiral and scroll waves in simulated and experimental cardiac tissue. New Journal of Physics 10, 125016.

Choi, H., Moin, P. \& Kim, J. 1994 Active turbulence control for drag reduction in wall-bounded flows. J. Fluid Mech. 262, 75-110.

Choi, H., Teman, R., Moin, P. \& Kim, J. 1993 Feedback control for unsteady flow and its application to the stochastic burgers equations. J. Fluid Mech. 50, 509-543.

Choi, J., Xu, C.-X. \& Sung, H. J. 2002 Drag reduction by spanwise wall oscillation in wall-bounded turbulent flows. AIAA Journal 255, 842-850.

Collis, S. S., Joslin, R. D., Seifert, A. \& Theofilis, V. 2004 Issues in active flow control: theory, control, simulation, and experiment. Prog. Aerosp. Sci. 40, 23789 .

Cottin-Bizonne, C., Cross, B., Steinberger, A. \& Charlaix, E. 2005 Boundary slip on smooth hydrophobic surfaces:intrinsic effects and possible artifacts. Phys. Rev. Lett. 94, 056102.

Cottin-Bizonne, C., Jurine, S., Baudry, J., Crassous, J., Restagno, F. \& Charlaix, E. 2002 Nanorheology: An investigation of the boundary condition at hydrophobic and hydrophilic interfaces. Eur. Phys. J. E 9, 47-53.

Crutchfield, J. P. \& Kaneko, K. 1988 Are attractors relevant to turbulence? Phys. Rev. Lett. 60, 2715-2718. 
Darbyshire, A. G. \& Mullin, T. 1995 Transition to turbulence in constant-massflux pipe flow. J. Fluid Mech. 289, 83-114.

Davidenko, J. M., Pertsov, A. V., Salomonsz, Remy, Baxter, W. \& JalIFE, J. 1992 Stationary and drifting spiral waves of excitation in isolated cardiac muscle. Nature 355, 349-351.

Davies, S. J. \& White, C. M. 1928 An experimental study of the flow of water in pipes of rectangular section. Phil. Trans. Roy. Soc. Lond. A 119, 92-107.

Del Álamo, J. C. \& Jiménez, J. 2009 Estimation of turbulent convection velocities and corrections to taylors approximation. J. Fluid Mech. 640, 5-26.

Drazin, P. G. A. \& ReId, W. H. 1981 Hydrodynamic Stability. Cambridge University Press.

Du, Y. \& Karniadakis, G. E. 2000 Suppressing wall turbulence by means of a transverse traveling wave. Science 288, 1230-1231.

Du, Y., Symeonidis, V. \& Karniadakis, G. E. 2002 Drag reduction in wallbounded turbulence via a transverse travelling wave. J. Fluid Mech. 457, 1-34.

Duguet, Y., Schlatter, P. \& Henningson, D. S. $2010 a$ Formation of turbulent patterns near the onset of transition in plane couette flow. J. Fluid Mech. 650, 119129.

Duguet, Y., Willis, A. P. \& Kerswell, R. R. $2010 b$ Slug genesis in cylindrical pipe flow. J. Fluid Mech. 663, 180-208.

Durst, F. \& Ünsal, B. 2006 Forced laminar to turbulent transition in pipe flows. J. Fluid Mech. 560, 449-464.

ECKERT, M. 2010 The troublesome birth of hydrodynamic stability theory: Sommerfeld and the turbulence problem. Eur. Phys. J. H 35, 29.

ECKhardt, B. \& FAisst, H. 2004 Dynamical systems and the transition to turbulence. In Laminar-turbulent transition and finite amplitude solutions (ed. T. Mullin \& R. R. Kerswell), pp. 35-50. Dordrecht: Springer.

Eckhardt, B., Schneider, T. M., Hof, B. \& Westerweel, J. 2007 Turbulence transition in pipe flow. Nonlinearity 39, 447-68.

Emmons, H. W. 1951 The laminar-turbulent transition in a boundary layer-part i. Journal of the Aeronautical Sciences (Institute of the Aeronautical Sciences) 18(7), 490-498.

FAisst, H. \& Eckhardt, B. 2004 Sensitive dependence on initial conditions in transition to turbulence in pipe flow. J. Fluid Mech. 504, 343-52.

FitzHugh, R. 1961 Impulses and physiological states in theoretical models of nerve membrane. Biophys. J. 1, 445-66. 
Fransson, J. H. M., Talamelli, A., Brandt, L. \& Cossu, C. 2006 Delaying transition to turbulence by a passive mechanism. Phys. Rev. Lett. 96, 064501.

Gibson, J. F., Halcrow, J. \& Cvitanović 2008 Visualizing the geometry of state space in plane couette flow. J. Fluid Mech. 611, 107-130.

Goldenfeld, N., Guttenberg, N. \& Gioia, G. 2010 Extreme fluctuations and the finite lifetime of the turbulent state. Phys. Rev. E 81, 035304.

Grossmann, S. 2000 The onset of shear flow turbulence. Rev. Mod. Phys. 72, 603618.

HaGen, G. 1839 Über die bewegung des wassers in engen cylindrischen röhren. Ann. Phys. Chem. 46, 423-42.

Gad-el HaK, M. 1996 Modern developments in flow control. Progress in Aerospace Sciences 49, 365-379.

Hamilton, J.M., Kim, J. \& Waleffe, F. 1995 Regeneration mechanisms of nearwall turbulence structures. J. Fluid Mech. 287, 317-348.

Henningson, D. S., Spalart, P. \& Kim, J. 1987 Numerical simulations of turbulent spots in plane poiseuille and boundary layer flow. Phys. Fluids 30, 2914.

HinRICHSEN, H. 2010 Non-equilibrium critical phenomena and phase transitions into absorbing states. Advances in Physics 49:7, 815-958.

Hodgkin, A. L. \& Huxley, A. F. 1952 A quantitative description of membrane current and its application to conduction and excitation in nerve. J. Physiol. 117, $500-544$.

Hof, B. 2004 Transition to turbulence in pipe flow. In Laminar-turbulent transition and finite amplitude solutions (ed. T. Mullin \& R. R. Kerswell), pp. 221-31. Dordrecht: Springer.

Hof, B., De Lozar, A., Avila, M., T, X. \& Schneider, T. M. 2010 Eliminating turbulence in spatially intermittent flows. Science 327, 1491-1494.

Hof, B., De Lozar, A., Kuik, D. J. \& Westerweel, J. 2008 Repeller or attractor? selecting the dynamical model for the onset of turbulence in pipe flow. Phys. Rev. Lett. 101, 214501.

Hof, B., Westerweel, J., Schneider, T. M. \& Eckhardt, B. 2006 Finite lifetime of turbulence in shear flows. Nature 443, 59-62.

Högberg, M., Bewley, T. \& Henningson, D. S. 2003 Linear feedback control and estimation of transition in plane channel flow. J. Fluid Mech. 481, 149-175.

Holzner, M., Song, B., Avila, M. \& Hof, B. 2013 Lagrangian approach to laminarturbulent interfaces. J. Fluid Mech. 723, 140-162. 
Hugues, Sandrine \& Randriamampianina, Anthony 1998 An improved projection scheme applied to pseudospectral methods for the incompressible navierstokes equations. Int. J. Num. Meth. Fluids 28, 501-521.

Itano, T. \& Toh, S. 2001 The dynamics of bursting process in wall turbulence. $J$. Phys. Soc. Japan 70, 703-16.

Iuso, G., Onorato, M., Spazzini, P. G. \& Cicca, G. M. D 2002 Wall turbulence manipulation by large-scale streamwise vortices. J. Fluid Mech. 473, 23-58.

Jimenez, J. 2013 How linear is wall-bounded turbulence. Phys. Fluids 25, 110814.

Jimenez, J. \& Moin, P. 1991 The minimal flow unit in near-wall turbulence. J. Fluid Mech. 225, 213-240.

Jimenez, J. \& Pinelli, A. 1999 The autonomous cycle of near-wall turbulence. J. Fluid Mech. 389, 335-359.

Joly, L., Ybert, C. \& Bocquet, L. 2006 Probing the nanohydrodynamics at liquid-solid interfaces using thermal motion. Phys. Rev. Lett. 96, 046101.

Joseph, P., Cottin-Bizonne, C., Benoi, J. M., Ybert, C., Journet, C., Tabeling, P. \& Bocquet, L. 2006 Slippage of water past superhydrophobic carbon nanotube forests in microchannels. Phys. Rev. Lett. 97, 156104.

Jovanovic, Jovan, Frohnapfel, Bettina, Srikantharajah, Rubitha, Jovanovic, Djordje, Lienhart, Hermann \& Delgado, Antonio 2011 Microflow-based control of near-wall fluctuations for large viscous drag reduction. Microfluid Nanofluid 11, 773-780.

Jung, Yong Chae. \& Bhushan, Bharat 2010 Biomimetic structures for fluid drag reduction in laminar and turbulent flows. J. Phys.: Condens. Matter 22, 035104 .

Kamrin, K., Bazant, M. Z. \& Stone, H. A. 2011 Effective slip boundary conditions for arbitrarily patterned surfaces. Phys. Fluids 23, 031701.

KAO, T. W. \& PARK, C. 1970 Experimental investigations of the stability of channel flows. part 1 . flow of a single liquid in a rectangular channel. J. Fluid Mech. 43, $145-164$.

Kasagi, N., Suzuki, Y. \& Fukagata, K. 2009 Microelectromechanical systemsbased feedback control of turbulence for skin friction reduction. Ann. Rev. Fluid Mech. 41, 231-51.

Kawahara, G., Uhlmann, M. \& van Veen, L. 2012 The significance of simple invariant solutions in turbulent flow. Ann. Rev. Fluid Mech. 44, 203-25.

KeRswell, R. R. 2005 Recent progress in understanding the transition to turbulence in a pipe. Nonlinearity 18, R17. 
Kim, J. 2003 Control of turbulent boundary layers. Phys. Fluids 15, 1093.

Kim, J. \& Bewley, T. R. 2007 A linear systems approach to flow control. Ann. Rev. Fluid Mech. 39, 383417.

Kim, J. \& Lim, Junwoo 2003 A linear mechanism in wall-bounded turbulent shear flows. Phys. Fluids 12, 1885-1888.

Kim, J., Moin, P. \& Moser, R. D. 1987 Turbulence statistics in fully developed channel flow at low reynolds number. J. Fluid Mech. 177, 133-166.

Kline, SJ, Reynolds, WC, Schraub, FA \& Runstadler, PW 1967 The structure of turbulent boundary layers. J. Fluid Mech. 30 (04), 741-773.

KNOBLOCH, E. 2008 Spatially localized structures in dissipative systems: open problems. Nonlinearity 21, T45-T46.

Kreilos, T. \& ECKhARDT, B. 2013 Symmetry related dynamics in parallel shear flows. arXiv arXiv:, 1309.4590v1 [physics.flu-dyn].

Kuik, D. J., Poelma, C. \& Westerweel, J. 2010 Quantitative measurement of the lifetime of localized turbulence in pipe flow. J. Fluid Mech. 645, 529-539.

Lee, C, Choi, C-H \& Kim, C-J 2008 Structured surfaces for a giant liquid slip. Phys. Rev. Lett. 101, 064501.

LEE, C \& KIm, C-J 2009 Maximizing the giant liquid slip on superhydrophobic microstructures by nanostructuring their sidewalls. Langmuir 25(21), 12812-12818.

Lee, C., Kim, J. \& Choi, H. 1998 Suboptimal control of turbulent channel flow for drag reduction. J. Fluid Mech. 358, 245-258.

Lieu, B. K., Moarref, R. \& Jovanovic, M. R. 2010 Controlling the onset of turbulence by streamwise travelling waves. part 1. direct numerical simulation. J. Fluid Mech. 663, 100-119.

LindGREn, E. R. 1969 Propagation velocity of turbulent slugs and streaks in transition pipe flow. Phys. Fluids 12, 418.

DE LozAr, A. \& Hof, B. 2010 Universality at the onset of turbulence in shear flows. arXiv pp. arXiv:1001.2481 [physics.flu-dyn].

Mellibovsky, F. \& Eckhardt, B. 2012 From travelling waves to mild chaos: a supercritical bifurcation cascade in pipe flow. J. Fluid Mech. 709, 149-190.

Meseguer, A. \& Trefethen, L.N. 2003 Linearized pipe flow to reynolds number 107. J. Comput. Phys. 186, 178-197.

Meseguer, A. \& Trefethen, L. N. 2000 A spectral petrov-galerkin formulation for pipe flow i: Linear stability and transient growth. Tech. Rep. 00/18. Oxford University Computing Laboratory. 
Michael, J. \& WALSh, J. 1983 Riblets as a viscous drag reduction technique. AIAA Journal 21, 485-486.

Min, TAegee \& Kim, John 2004 Effects of hydrophobic surface on skin-friction drag. Phys. Fluids 16, L55.

Monrref, R. \& Jovanovic, M. R. 2010 Controlling the onset of turbulence by streamwise travelling waves. part 1. receptivity analysis. J. Fluid Mech. 663, 70-99.

Moin, P. \& Mahesh, K. 1998 Direct numerical simulation: a tool in turbulence research. Ann. Rev. Fluid Mech. 20, 539-78.

Moxey, D. \& BARkley, D. 2010 Distinct large-scale turbulent-laminar states in transitional pipe flow. PNAS 107, 8091-8096.

Müller, S. C., Plesser, T. \& Hess, B. 1985 The structure of the core of the spiral wave in the belousov-zhabotinskii reaction. Science 230, 661-663.

Mullin, T. 2011 Experimental studies of transition to turbulence in a pipe. Ann. Rev. Fluid Mech. 43, 1-24.

Mullin, T. \& Peixinho, J. 2006 Recent observations in the transition to turbulence in a pipe. In IUTAM Symposium on laminar-turbulent transition (ed. R. Govindarajan), p. 45. Bangalore: Springer.

Murray, J. D., Stanley, E. A. \& Brown, D. L. 1986 On the spatial spread of rabies among foxes. Phil. Trans. Roy. Soc. Lond. B 229, 111-150.

NAGATA, M. 1990 Three-dimensional finite-amplitude solutions in plane couette flow : bifurcation from infinity. J. Fluid Mech. 217, 519-527.

Nagumo, J., Arimoto, S. \& Yosizawa, S. 1962 An active pulse transmission line simulating nerve axon. Proceedings of the TRE 50, 2061.

Niemela, J. J., Ahler, G. \& Cannell, D. S. 1990 Localized traveling waves in binary-fluid convection. Phys. Rev. Lett. 64, 1365.

Nishi, M., Ünsal, B., Durst, F. \& Biswas, G. 2008 Laminar-to-turbulent transition of pipe flows through puffs and slugs. J. Fluid Mech. 614, 425-446.

Orszag, S. A. \& Kells, L, C. 1980 Transition to turbulence in plane poiseuille and plane couette flow. J. Fluid Mech. 96, 159-205.

Orszag, S. A. \& Patterson, G. S. 1971 Numerical simulation of threedimensional homogeneous isotropic turbulence. Phys. Rev. Lett. 28, 76-79.

PARK, H, PARK, H \& Kim, J 2013 A numerical study of the effects of superhydrophobic surface on skin-friction drag in turbulent channel flow. Phys. Fluids 25, 110815.

Pei, J., Chen, J., She, Z.-S. \& Hussain, F. 2012 Model for propagation speed in turbulent channel floas. Phys. Rev. E 86, 046307. 
Peixinho, J. \& Mullin, T. 2006 Decay of turbulence in pipe flow. Phys. Rev. Lett. 96, 094501.

Pfenniger, W. 1961 Transition in the inlet length of tubes at high reynolds numbers. In Boundary Layer and flow control (ed. G. V. Lachman), pp. 970-980. Pergamon, Oxford.

Poiseuille, J. L. M. 1840 Recherches expérimentales sur le mouvement des liquides dans les tubes de très petit diamètres. C. R. Acad. Sci. 11, 961-67.

Qu, J. \& Rothstein, J. P. 2005 Direct velocity measurements of the flow past drag-reducing ultrahydrophobic surfaces. Phys. Fluids 17, 103606.

Quadrio, M., Ricco, P. \& Viotti, C. 2009 Streamwise-travelling waves of spanwise wall velocity for turbulent drag reduction. J. Fluid Mech. 627, 161-178.

Reddy, S. \& Henningson, D. S. 1993 Energy growth in viscous channel flows. J. Fluid Mech. 252, 209-238.

REYNOLDS, O. 1883a An experimental investigation of the circumstances which determine whether the motion of water shall be direct or sinuous and of the law of resistance in parallel channels. Phil. Trans. Roy. Soc. Lond. A 35, 84-99.

REYNOLDS, O. $1883 b$ An experimental investigation of the circumstances which determine whether the motion of water shall be direct or sinuous and of the law of resistance in parallel channels. Phil. Trans. Roy. Soc. Lond. A 174, 935-982.

REynolds, O. 1895 On the dynamical theory of incompressible viscous fluids and the determination of the criterion. Phil. Trans. Roy. Soc. Lond. A 186, 123-164.

Richter, R. \& Barashenkov, I. V. 2005 Two-dimensional solitons on the surface of magnetic fluids. Phys. Rev. Lett. 94, 184503.

Robinson, S. K. 1991 Coherent motions in the turbulent boundary layers. Ann. Rev. Fluid Mech. 23, 601-39.

RoтtA, J. 1956 Experimenteller beitrag zur entstehung turbulenter strömung im rohr. Ing.-Arch 24, 258-82.

Ruelle, D. \& Takens, F. 1971 On the nature of turbulence. Comm. Math. Phys. 20, 167-192.

Samanta, D., DE Lozar, A. \& Hof, B. 2011 Experimental investigation of laminar turbulent intermittency in pipe flow. J. Fluid Mech. 681, 193204.

Schmid, P. J \& Henningson, D. S. 1994 Optimal energy density growth in hagenpoiseuille flow. J. Fluid Mech. 277, 197-225.

Schoppa, W. \& Hussain, F. 1998 A large-scale control strategy for drag reduction in turbulent boundary layers. Phys. Fluids 10, 1049. 
Schoppa, W. \& Hussain, F. 2002 Coherent structure generation in near-wall turbulence. J. Fluid Mech. 453, 57-108.

Semeraro, O., Bagheri, S., Brandt, L. \& Henningson, D. S. 2011 Feedback control of three-dimensional optimal disturbances using reduced-order models. J. Fluid Mech. 677, 63-102.

Semeraro, O., Bagheri, S., Brandt, L. \& Henningson, D. S. 2013 Transition delay in a boundary layer flow using active control. J. Fluid Mech. 731, 288-311.

Sirovich, L. \& Karlsson, S. 1997 Turbulent drag reduction by passive mechanisms. Nature 388, 753-755.

Song, B. \& Hof, B. 2014 Deterministic and stochastic aspects of the transition to turbulence. J. Stat. Mech. 2014, P02001.

Sreenivasan, K. R. \& Ramshankar, R. 1986 Transition intermittency in open flows, and intermittency routes to chaos. Physica 23D, 246-258.

Stone, H. A., Stroock, A. D. \& Ajdari, A. 2004 Engineering flows in small devices. Ann. Rev. Fluid Mech. 36, 381-411.

Sumitani, Y. \& Kasagi, N. 1995 Direct numerical simulation of turbulent transport with uniform wall injection and suction. AIAA J. 33, 12201228.

TAYLOR, G. I. 1935 The transport of vorticity and heat through fluids in turbulent motion. Phil. Trans. Roy. Soc. Lond. A 135, 685.

Tennekes, H. \& Lumley, J. L. 1972 A first course in turbulence. The MIT Press.

Tlidi, M., Mandel, P. \& Haelterman, M. 1997 Spatiotemporal patterns and localized structures in nonlinear optics. Phys. Rev. E 56, 6524.

Tlidi, M., Mandel, P. \& Lefever, R. 1994 Localized structures and localized patterns in optical bistability. Phys. Rev. Lett. 73, 640.

Trefethen, L. N., Trefethen, A. E., Reddy, S. C. \& Driscoll, T. A. 1993 Hydrodynamic instability without eigenvalues. Science 261, 578-584.

Tuerke, F. \& Jimenez, J. 2013 Simulations of turbulent channels with prescribed velocity profiles. J. Fluid Mech. 723, 587-603.

Tyson, J. J. \& Keener, J. P. 1988 Singular perturbation theory of traveling waves in excitable media (a review). Physica D 32, 327-361.

Vinogradova, O. I. 1999 Slippage of water over hydrophobic surfaces. Int. J. Min. Process 56, 31-60.

Waleffe, F. 1997 On a self-sustaining process in shear flows. Phys. Fluids 9, 883900. 
WALeffe, F. 1998 Three-dimensional coherent states in plane shear flows. Phys. Rev. Lett. 81(19), 4140-43.

Waleffe, F. 2001 Exact coherent structures in channel flow. J. Fluid Mech. 435, 93-102.

Waleffe, F. 2003 Homotopy of exact coherent structures in plane shear flows. Phys. Fluids 15, 1517-34.

Willis, A. P., Cvitanović, P. \& Avila, M. 2013 Revealing the state space of turbulent pipe flow by symmetry reduction. J. Fluid Mech. 721, 514-540.

Willis, A. P. \& Hwang, Y. 2010 Optimally amplified large-scale streaks and drag reduction in turbulent pipe flow. Phys. Rev. E 82, 036321.

Willis, A. P. \& Kerswell, R. R. 2007 Critical behavior in the relaminarization of localized turbulence in pipe flow. Phys. Rev. Lett. 98, 014501.

Willis, A. P. \& Kerswell, R. R. 2009 Turbulent dynamics of pipe flow captured in a reduced model: puff relaminarisation and localised 'edge' states. J. Fluid Mech. 619, 213-233.

Winfree, A. T. 1972 Spiral waves of chemical activity. Science 175, 634-636.

Wygnanski, I, Sokolov, M. \& Friedman, D. 1976 On a turbulent 'spot' in a laminar boundary layer. J. Fluid Mech. 78, 785-819.

Wygnanski, I. J. \& Champagne, F. H. 1973 On transition in a pipe. part 1. the origin of puffs and slugs and the flow in a turbulent slug. J. Fluid Mech. 59, $281-335$.

Wygnanski, I. J., Sokolov, M. \& Friedman, D. 1975 On transition in a pipe. part 2. the equilibrium puff. J. Fluid Mech. 69, 283-304.

Xu, C.-X., Choi, J. \& Sung, H. J. 2002 Suboptimal control for drag reduction in turbulent pipe flow. Fluid Dynam. Res. 30, 217-231.

Zaikin, A. N. \& Zhabotinskis, A. M. 1970 Concentration wave propagation in two-dimensinonal liquid-phase self-oscillating system. Nature 225, 535-537.

Zhou, J., Adrian, R. J., Balachandar, S. \& Kendall, T. M. 1999 Mechanisms for generating coherent packets of hairpin vortices in channel flow. J. Fluid Mech. 387, 353-396.

Zhu, Y. \& Granick, S. 2002 Limits of the hydrodynamic no-slip boundary condition. Phys. Rev. Lett. 88, 106102. 


\section{Curriculum Vitae}

\section{BAOFANG SONG}

Date of birth: July 20, 1985

Max Planck Institute for Dynamics and Self-Organization

Gerog-August-Universität Göttingen

Current address:

Institute of Science and Technology Austria (IST Austria),

Am Campus 1, 3400 Klosterneuburg, Austria

+4322439000 5808, bsong@ist.ac.at

\section{Professional experience}

Research Assistant

October 2013 to present

Institute of Science and Technology Austria (IST Austria), Am Campus 1, 3400 Klosterneuburg, Austria

- Performed DNS study on the transition to fully turbulent flow in pipes

- Guided a first-year PhD student on his rotation project "Transient Growth in Channel Flow"

Research Assistant

October 2010 to September 2013

Max Planck Institute for Dynamics and Self-Organization, Am Fassberg 17, 37077 Göttingen, Germany

- Developed turbulence control strategies in pipe flow

- Implemented a Lagrangian approach to investigate the local dynamics on the laminar/turbulent interfaces

- Wrote parallel DNS channel flow and pipe flow codes with Fortran90 and MPI

- Examined the influence of partial slip boundary condition on turbulence in pipes

- Searched for localized exact solutions in Plane Poiseuille and pipe flow

\section{Education}

PhD Candidate, Physics,

Expected in September 2014

Max Planck Institute for Dynamics and Self-Organization, Am Fassberg 17, 37077 Göttingen, Germany 
- PhD project: Direct numerical simulation of transition to turbulence and turbulence control in pipe flow. Supervisor: Prof. Björn Hof.

- Hold a Chinese Scholarship Fund (10.2010-09.2013), Max Planck Fellowship (09.2013-05.2014)

Master of Engineering, Computational Fluid Dynamics,

April 2010

Department of Fluid Dynamics, School of Aeronautics, Northwestern Polytechnical University, 710072 Xi'an, China

- Thesis: Numerical Analysis of the Effects of Wings Oscillation on its Aerodynamic Characteristics in Wind Tunnel Tests. Supervisor: Prof. Zhengyin Ye

- GPA $3.77 / 4$

Bachelor of Engineering, Aeronautical Engineering, July 2007

School of Aeronautics, Northwestern Polytechnical University, 710072 Xi'an, China

- Thesis: Numerical Simulations of the Transonic Unsteady Flow Around an Airfoil. Supervisor: Prof. Zhengyin Ye

- Outstanding graduate of the year 2007

- Outstanding Bachelor's Thesis of the School of Aeronautics

- GPA 3.5/4, top $3 \%$

\section{Publications}

1. Song B., Willis A. P., Hof B., \& Avila M. Relaminarisation of fully turbulent pipe flow by manipulation of the mean shear, under preparation

2. Song B., Kühnen J., Willis A. P., Avila M., \& Hof B. Relaminarisation of fully turbulent pipe flow, under preparation

3. Barkley D., Song B., Vasudevan M., Avila M., \& Hof B. Emergence of fully turbulent flow, submitted to Science

4. Song B. \& Hof B. Deterministic and stochastic aspects of the transition to turbulence, J. Stat. Mech. (2014), P02001

5. Holzner M., Song B., Avila M., \& Hof B. Lagrangian approach to laminar-turbulent interfaces in transitional pipe flow, J. Fluid Mech. (2013) 723, 140-162.

6. Song B. \& Ye Z., 2011 Numerical analysis of the effects of wings oscillation on its aerodynamic characteristics in wind tunnel tests, ACTA AERODYNAMICA SINICA 29(2), 226-230 


\section{Conference presentations}

1. "A passive control mechanism that relaminarises fully turbulent pipe flow", at Euromech Colloquium EC565 on Subcritical Transition to Turbulence, Corsica, France, May $6^{\text {th }}-9^{\text {th }}, 2014$

2. "Relaminarising fully turbulent pipe flow", on the $14^{\text {th }}$ European Turbulence Conference, Lyon, France, September $1^{\text {st }}-4^{\text {th }}, 2013$

3. "Relaminarization of Turbulence in Pipe Flow", on the $23^{\text {rd }}$ International Congress of Theoretical and Applied Mechanics, Beijing, China. August $19^{\text {th }}-24^{\text {th }}, 2012$

4. "The spreading of turbulence in pipe flow", on the $5^{\text {th }}$ European Postgraduate Fluid Dynamics Conference, Göttingen, Germany. August $8^{t h}-12^{\text {th }}, 2011$

\section{Patents}

Turbulence relaminarisation mechanism (PCT/EP2014/055329)

\section{Teaching experience}

1. Student tutor in Analytical Mechanics (BSc Physics), winter semester 2012/2013, Gerog-August-Universität Göttingen

2. Student tutor in Computer-Aided Scientific Computing (BSc Physics), summer semester 2012, Gerog-August-Universität Göttingen

\section{Skills}

- Proficient in Fortran90, Matlab, and MPI parallel programming

- Familiar with c/c++ and Bourne shell programming

- Extensive experience with Linux system and High Performance Computers

- Good English presentational skills and English writing skills 